AP A V, E R
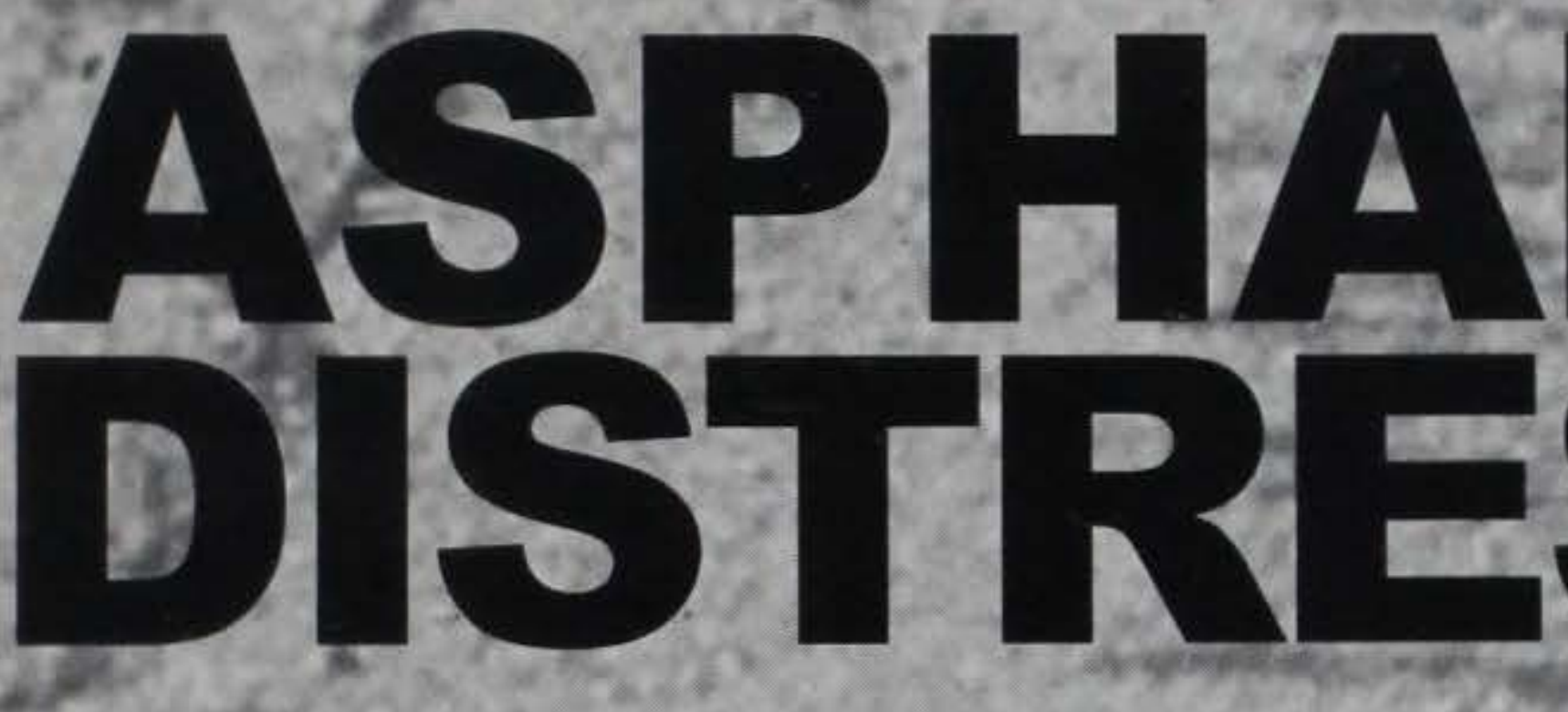

\title{
M A
}
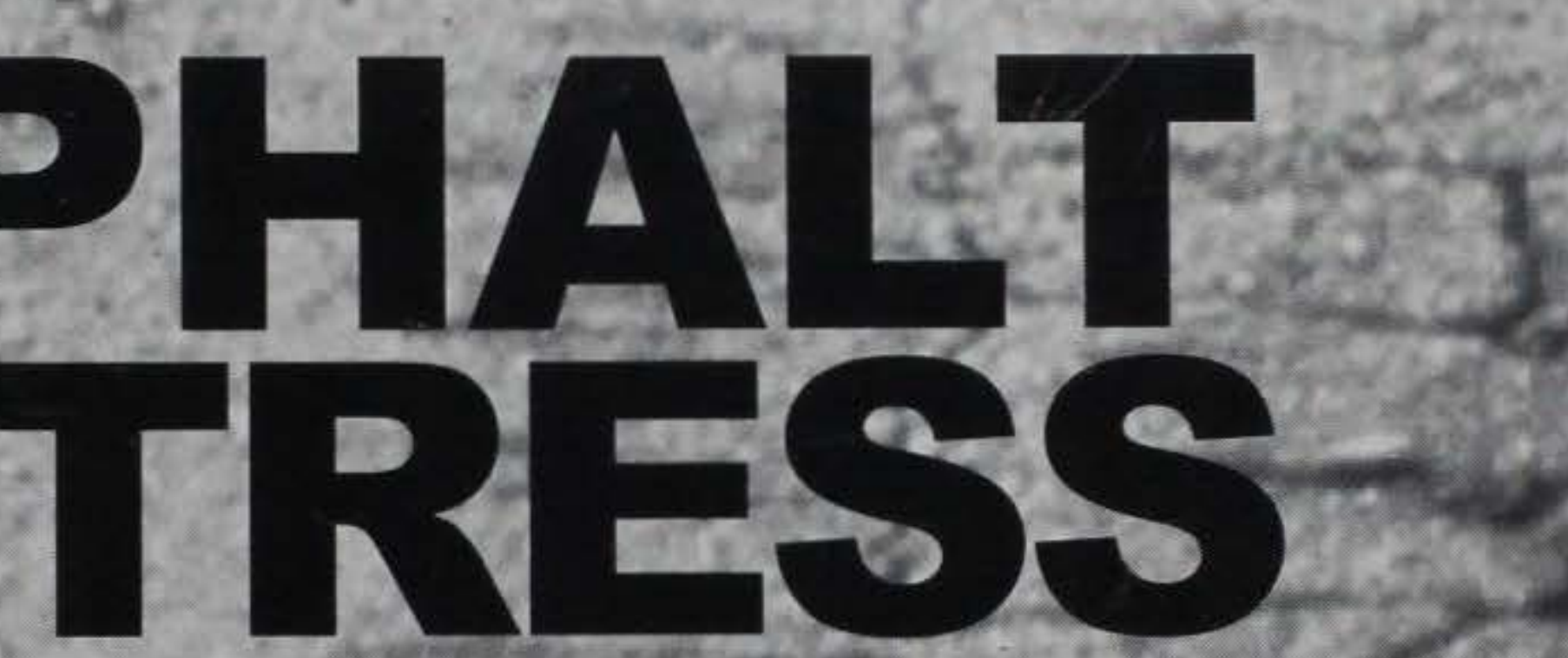

$-1$
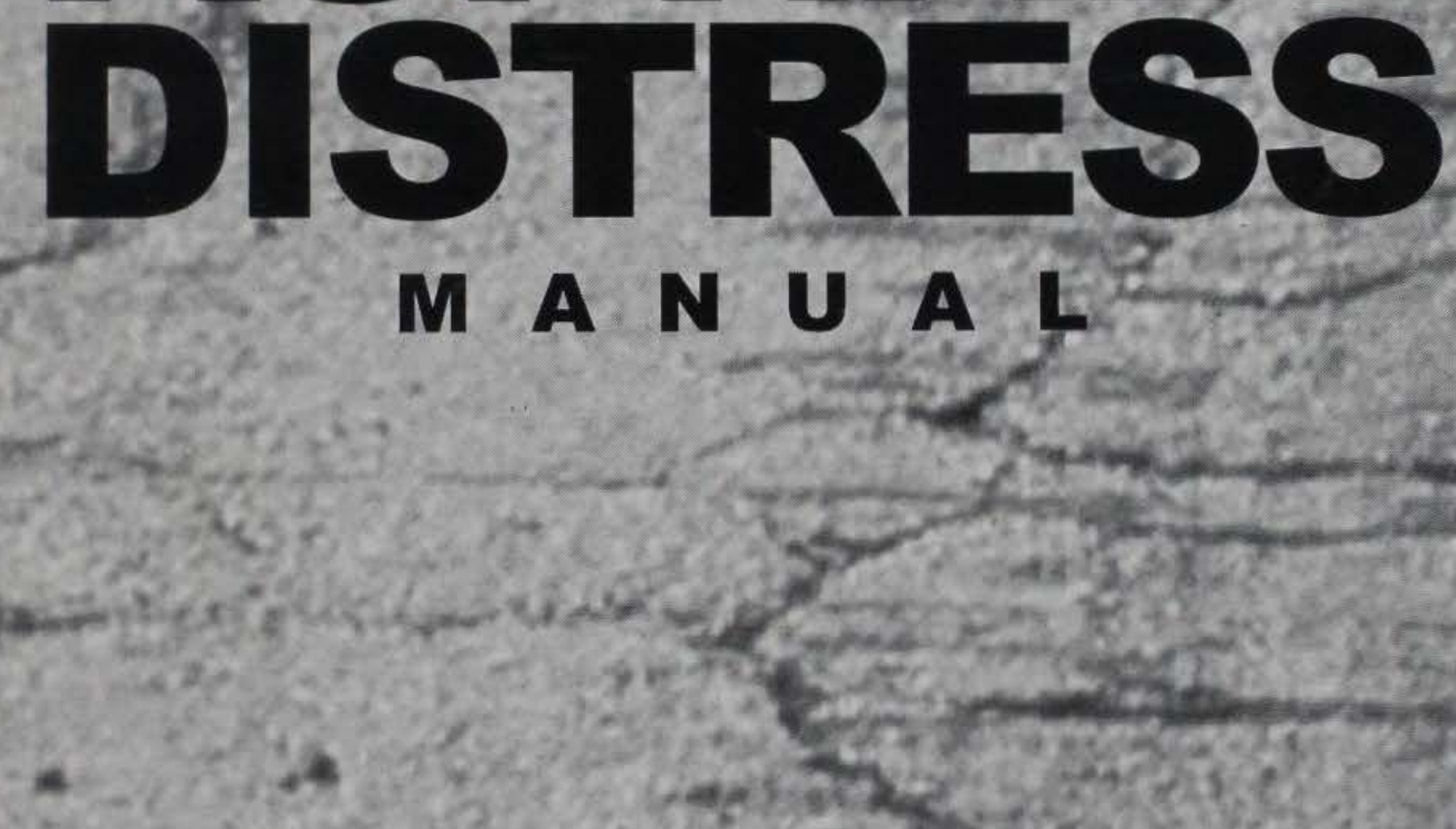

\section{Pavement Distress: \\ Z Identification Guide} FOR ASPHALT-SURFACED

ROADS AND PARKING LOTS

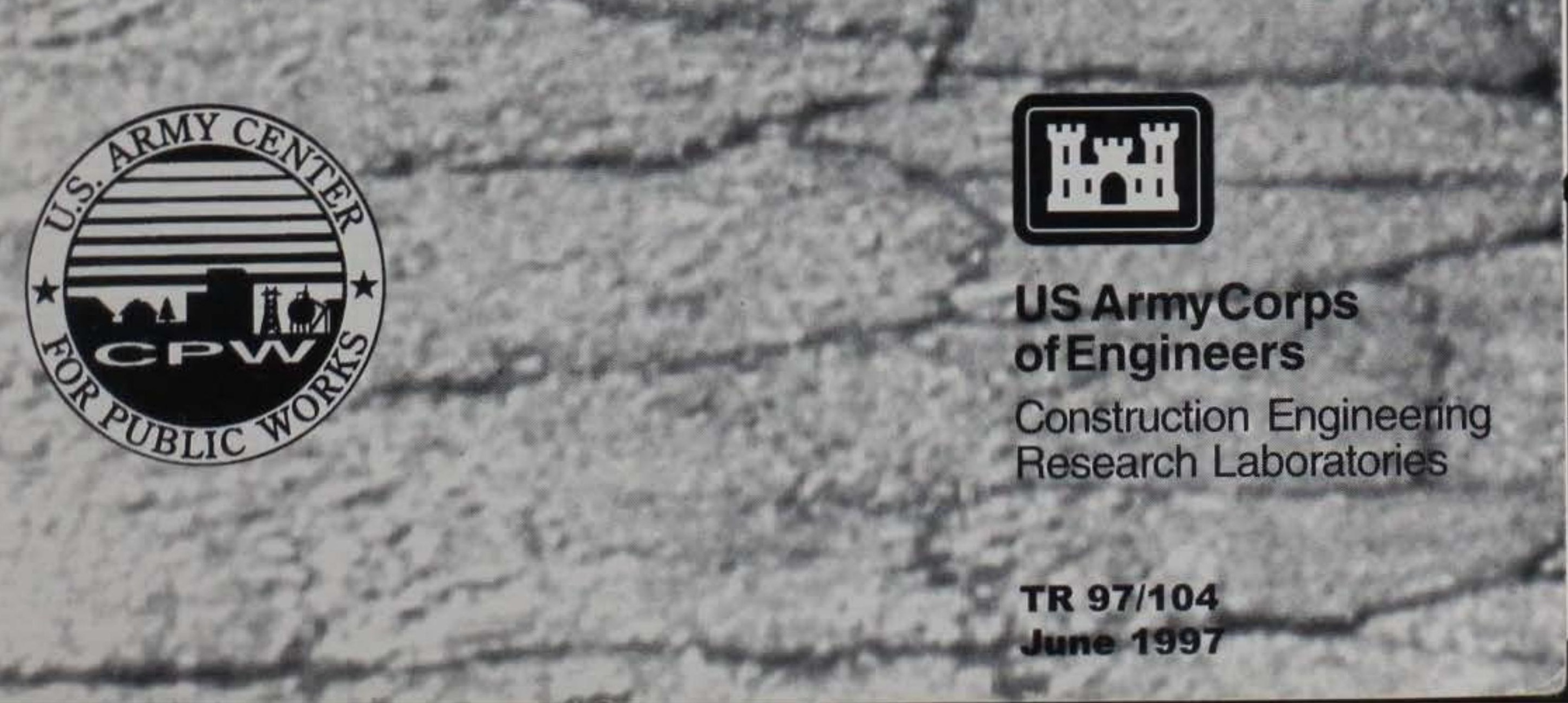




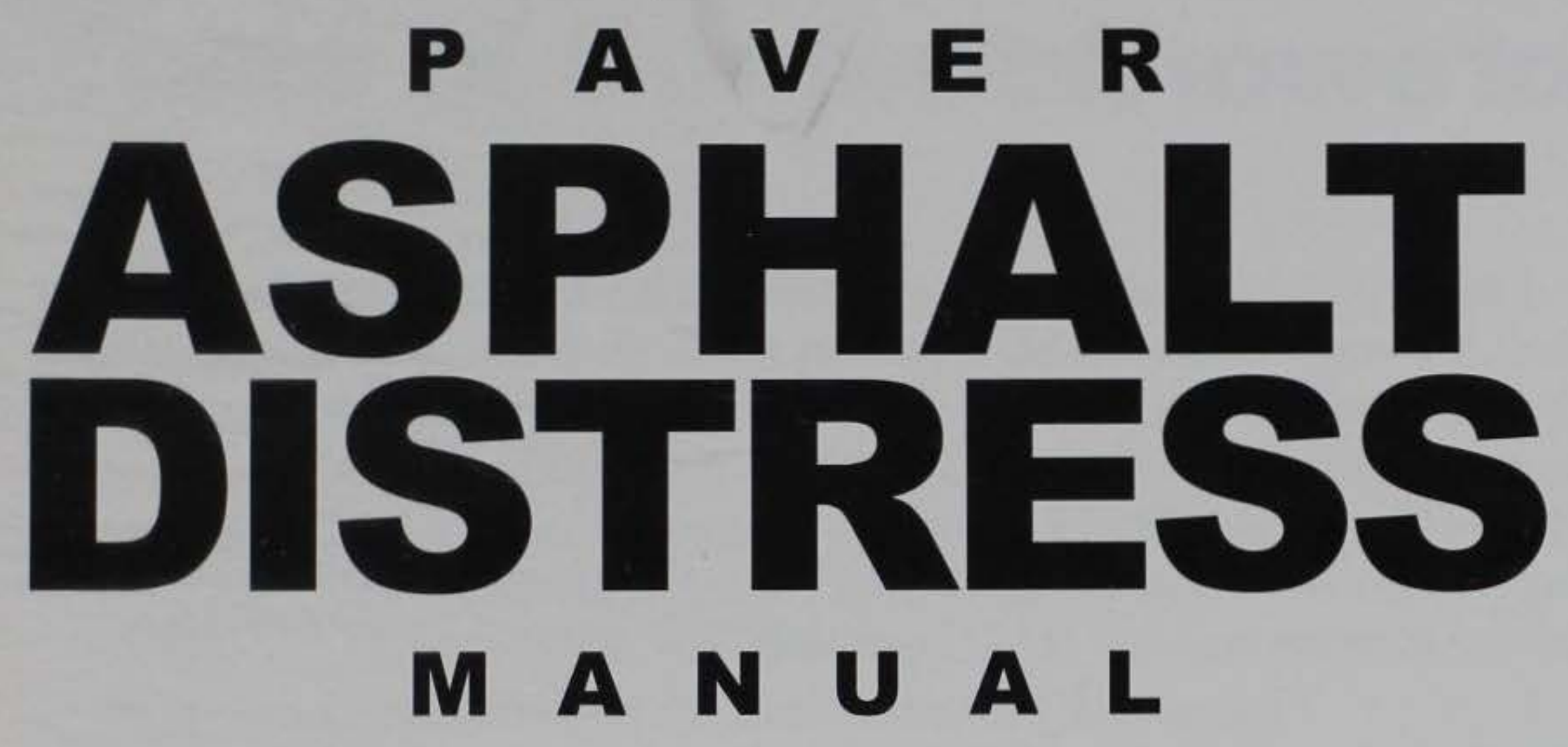

\author{
Pavement Distress \\ IDENTIFICATION GuIDE \\ for Asphalt-SURfaced \\ Roads and Parking Lots
}
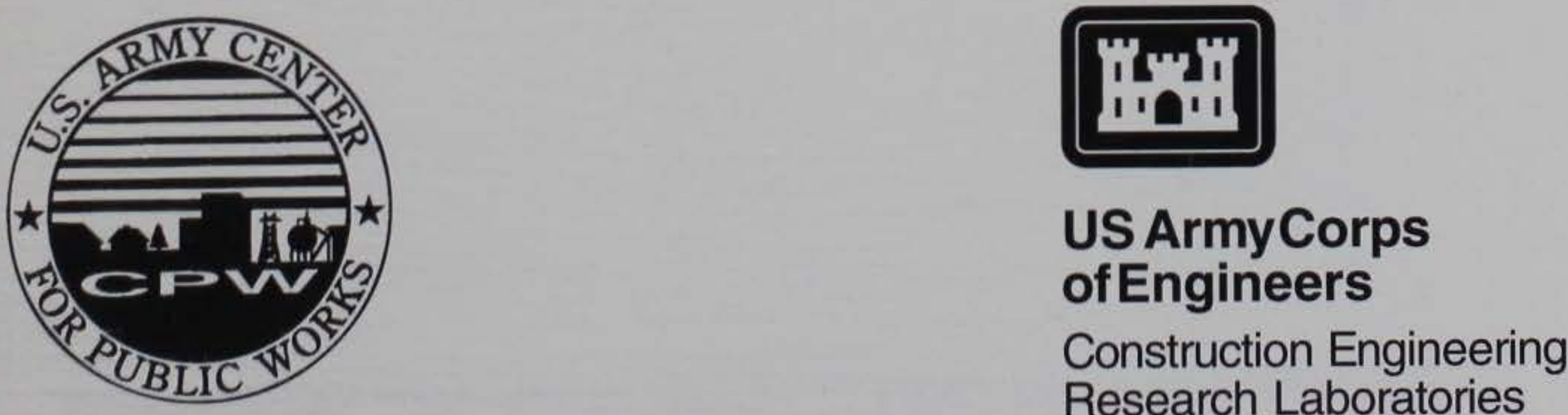

Research Laboratories 


\section{Foreword}

This document is a revision and technical update of the original PAVER Asphalt Distress Manual by M.Y. Shahin and S.D. Kohn (USACERL, June 1989). This update was prepared for the Directorate of Engineering, U.S. Army Center for Public Works (USACPW) under Military Interdepartmental Purchase Request (MIPR) E87950430, "Technical Support for Army Transportation Infrastructure (PAVER)," dated September 1995. The Technical Monitor was Ali A. Achmar, CECPW-ER.

The work was conducted by the Maintenance Management and Preservation Division (FL-P) of the Facilities Technology Laboratory (FL), U.S. Army Construction Engineering Research Laboratories (USACERL). The Principal Investigator was M.Y. Shahin, CECER-FL-P. Jeffrey A. Burkhalter is acknowledged for his assistance in collecting new data, revising technical content, and preparing the revised distress manual. Gordon L. Cohen, Technical Information Team (CECER-TR-I), was publication designer and technical editor. Dr. Simon S. Kim is Chief, CECER-FL-P, and Donald F. Fournier is Acting Operations Chief, CECER-FL.

Dr. Michael J. O'Connor is the Director of USACERL. 


\section{Table of Contents}

Foreword

\section{Introduction}

Objective and Scope of This Manual

Identifying and Counting Asphalt Pavement Distresses

Ride Quality Assessment

The Asphalt Pavement Distresses

Alligator Cracking (Fatigue)

Bleeding

Block Cracking

Bumps and Sags

Corrugation

Depression

Edge Cracking

Joint Reflection Cracking

Lane/Shoulder Drop-Off

Longitudinal and Transverse Cracking

Patching and Utility Cut Patching

Polished Aggregate

Potholes

Railroad Crossing

Rutting

Shoving

Slippage Cracking

Swell

Weathering and Raveling 


\section{Objective and Scope of This Manual}

This pavement distress manual is intended for use in conjunction with the PAVER Engineered Management System (EMS). The manual defines distresses and measurement methods for inspecting the condition of asphalt-surfaced roads and parking lots. The definitions and methods are keyed for use in determining a Pavement Condition Index (PCI), which is a central component of the PAVER EMS.

This manual is specially designed for use in the field during pavement inspections, but is also appropriate for use in pavement inspection training courses. A reference list is provided on page 48 for readers who want more detailed information about PAVER, determining the PCI for roads and parking lots, and how to use it in pavement management.

\section{Identifying and Counting Asphalt Pavement Distresses}

New PAVER users often ask about identification and counting methods for various distresses. Detailed answers to these questions are included for each distress under the heading "How to Count." For the reader's convenience, however, the most frequently raised issues are addressed below:

1. Alligator cracking. If alligator cracking and rutting occur in the same area, each is recorded separately at its respective severity level.

2. Bleeding with polished aggregate. If bleeding is counted, polished aggregate is not counted in the same area.

3. Spalling. In PAVER, spalling is defined as the further breaking of asphalt or loss of materials around cracks or joints.

4. Crack severity. If a crack varies in severity along its entire length, each portion showing an identifiable level should be recorded individually. If the different severity levels cannot easily be divided into distinct portions, the crack should be rated at the highest severity level present.

5. Distresses within patches. Distresses (including cracking and potholes) found in a patched area are not recorded separately. The effect of the distress on the patch, however, is taken into consideration when determining the severity level of the patch. 
6. Polished aggregate. A significant amount of polished aggregate should be present before it is counted.

7. Raveling. A distress is said to be raveled if the area surrounding the distress is broken (sometimes to the extent that pieces are missing).

The reader should note that the items above are general issues, and do not stand alone as inspection criteria. To measure each distress type properly, the inspector must be familiar with the individual distress criteria, which are described and illustrated on the pages that follow.

\section{Ride Quality Assessment}

Ride quality must be assessed in order to establish a severity level for the following distress types:

- Bumps

- Corrugation

- Railroad crossings
- Shoving

- Swells

To assess ride quality for these distresses, the inspector should use the following severity-level definitions:

L Low. Vehicle vibrations (e.g., from corrugation) are noticeable, but no reduction in speed is necessary for comfort or safety; and/or individual bumps or settlements cause the vehicle to bounce slightly, but create little discomfort.

M Medium. Vehicle vibrations are significant and some reduction in speed is necessary for safety and comfort; and/or individual bumps or settlements cause the vehicle to bounce significantly, creating some discomfort.

H High. Vehicle vibrations are so excessive that speed must be reduced considerably for safety and comfort; and/or individual bumps or settlements cause the vehicle to bounce excessively, creating substantial discomfort, safety hazard, or high potential vehicle damage.

The inspector should drive at the posted speed in a sedan that is representative of cars typically seen in local traffic. Pavement sections near stop signs should be rated at a deceleration speed appropriate for the intersection. 


\section{The Asphalt Pavement Distresses}

The following pages describe and illustrate the 19 distresses defined in the PAVER Engineered Management System (EMS) for asphalt-surfaced roads and parking lots.

Each two-page spread is dedicated to a single type of asphalt distress. The distresses are presented alphabetically and numbered. The pages of the manual are tab-indexed for the user's convenience during inspections in the field. 


\section{Alligator Cracking (Fatigue)}

\section{Description}

Alligator or fatigue cracking is a series of interconnecting cracks caused by fatigue failure of the asphalt concrete surface under repeated traffic loading. Cracking begins at the bottom of the asphalt surface (or stabilized base) where tensile stress and strain are highest under a wheel load. The cracks propagate to the surface initially as a series of parallel longitudinal cracks. After repeated traffic loading, the cracks connect, forming many sided, sharp-angled pieces that develop a pattern resembling chicken wire or the skin of an alligator. The pieces are generally less than $0.5 \mathrm{~m}(1.5 \mathrm{ft})$ on the longest side. Alligator cracking occurs only in areas subjected to repeated traffic loading, such as wheel paths. (Pattern-type cracking that occurs over an entire area not subjected to loading is called "block cracking," which is not a load-associated distress.)

\section{Severity Levels}

L Fine, longitudinal hairline cracks running parallel to each other with no, or only a few interconnecting cracks. The cracks are not spalled (Figure 1a).

M Further development of light alligator cracks into a pattern or network of cracks that may be lightly spalled (Figure 1b).

H Network or pattern cracking has progressed so that the pieces are well defined and spalled at the edges. Some of the pieces may rock under traffic (Figure 1c).

\section{How to Measure}

Alligator cracking is measured in square meters (feet) of surface area. The major difficulty in measuring this type of distress is that two or three levels of severity often exist within one distressed area. If these portions can be easily distinguished from each other, they should be measured and recorded separately. However, if the different levels of severity cannot be divided easily, the entire area should be rated at the highest severity present. If alligator cracking and rutting occur in the same area, each is recorded separately as its respective severity level. 


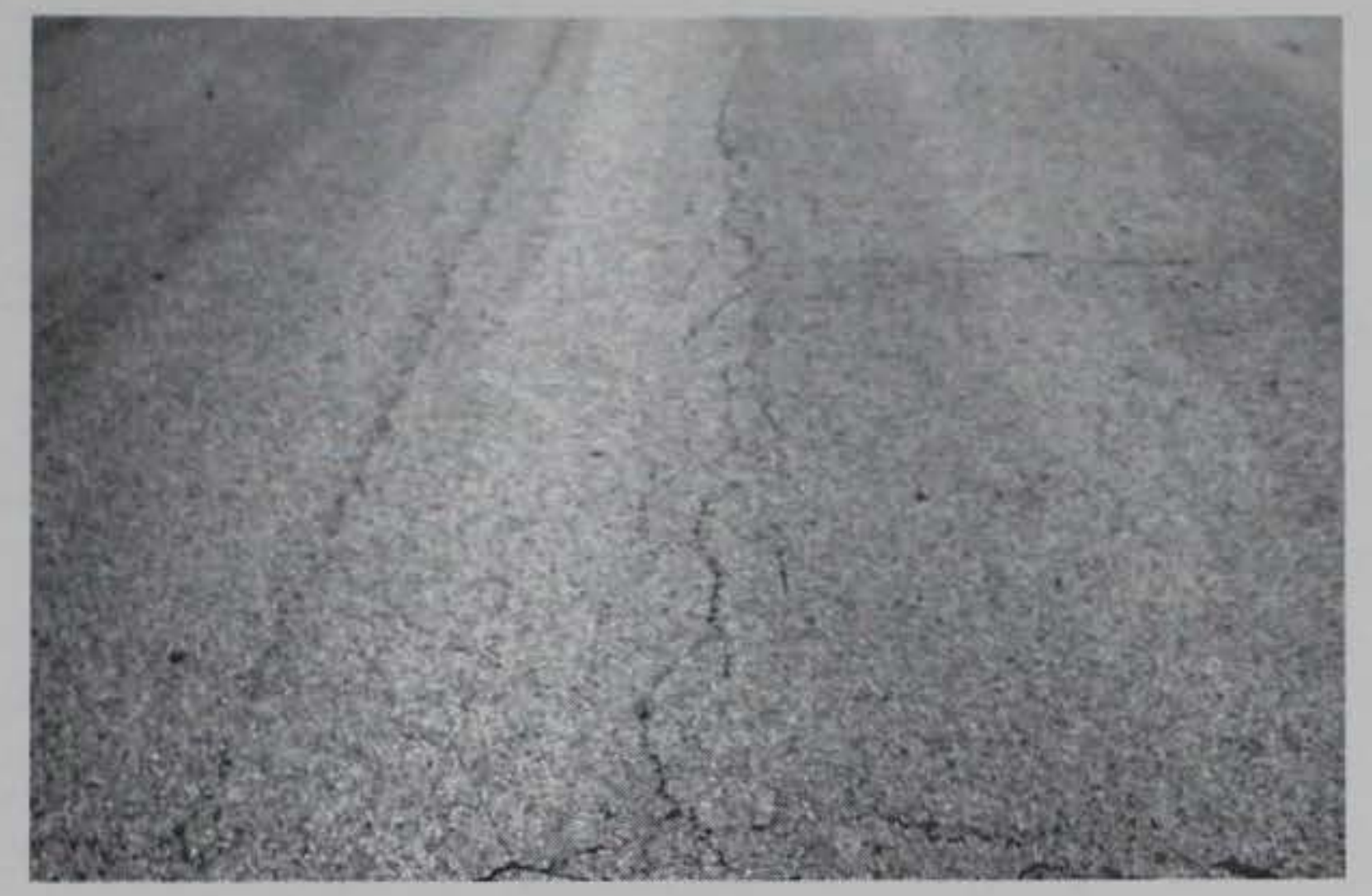

Figure 1a. Low-Severity Alligator Cracking.

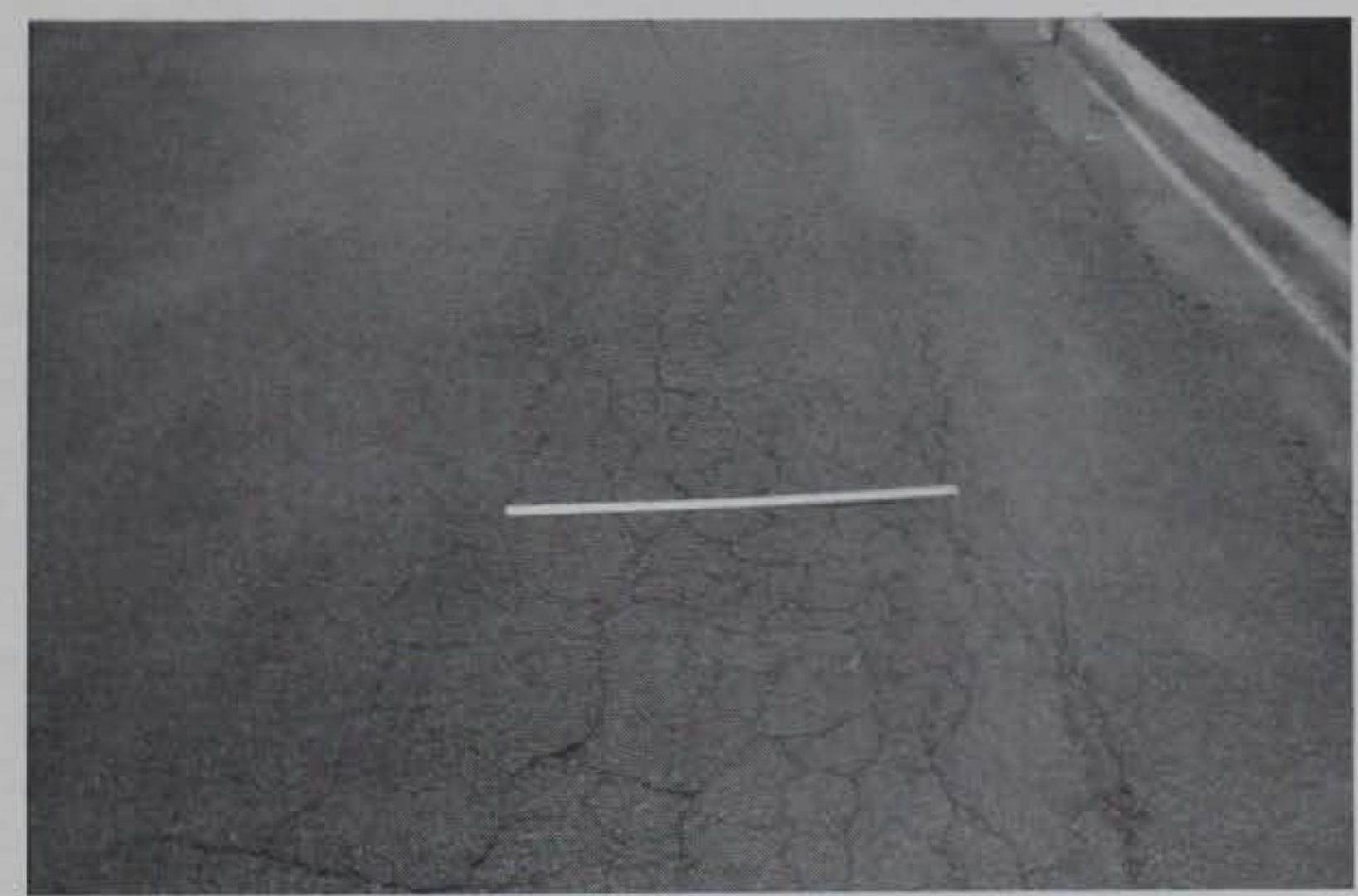

Figure 1b. Medium-Severity Alligator Cracking.

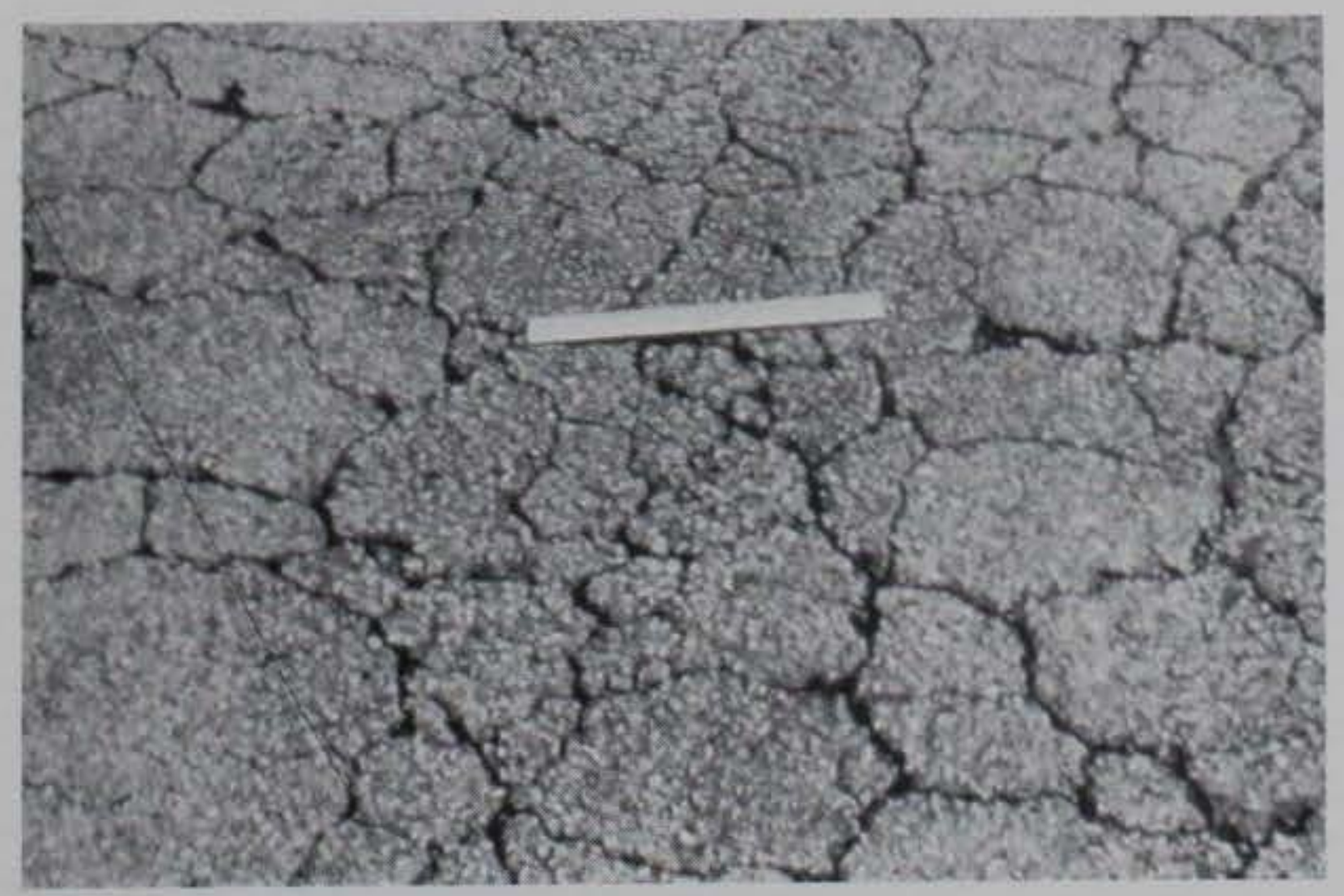

를

Figure 1c. High-Severity Alligator Cracking. 


\section{Bleeding}

\section{Description}

Bleeding is a film of bituminous material on the pavement surface that creates a shiny, glasslike, reflecting surface that usually becomes quite sticky. Bleeding is caused by excessive amounts of asphaltic cement or tars in the mix, excess application of a bituminous sealant, and/or low air void content. It occurs when asphalt fills the voids of the mix during hot weather and then expands onto the pavement surface. Since the bleeding process in not reversible during cold weather, asphalt or tar will accumulate on the surface.

\section{Severity Levels}

L Bleeding has only occurred to a very slight degree and is noticeable only during a few days of the year. Asphalt does not stick to shoes or vehicles (Figure 2a).

M Bleeding has occurred to the extent that asphalt sticks to shoes and vehicles during only a few weeks of the year (Figure 2b).

H Bleeding has occurred extensively and considerable asphalt sticks to shoes and vehicles during at least several weeks of the year (Figure 2c).

\section{How to Measure}

Bleeding is measured in square meters (feet) of surface area. If bleeding is counted, polished aggregate should not be counted. 


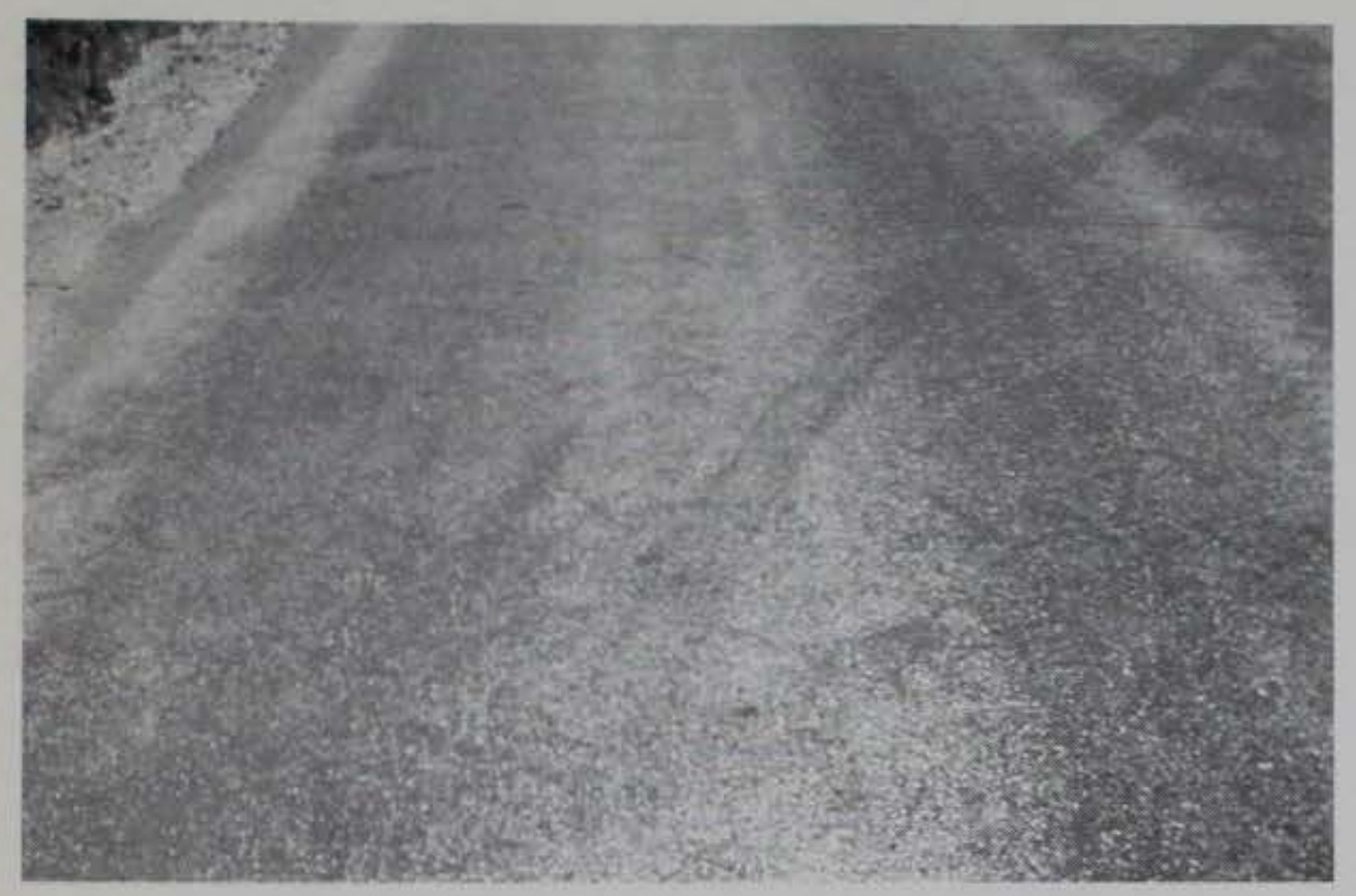

Figure 2a. Low-

Severity Bleeding.

Figure 2b. MediumSeverity Bleeding.
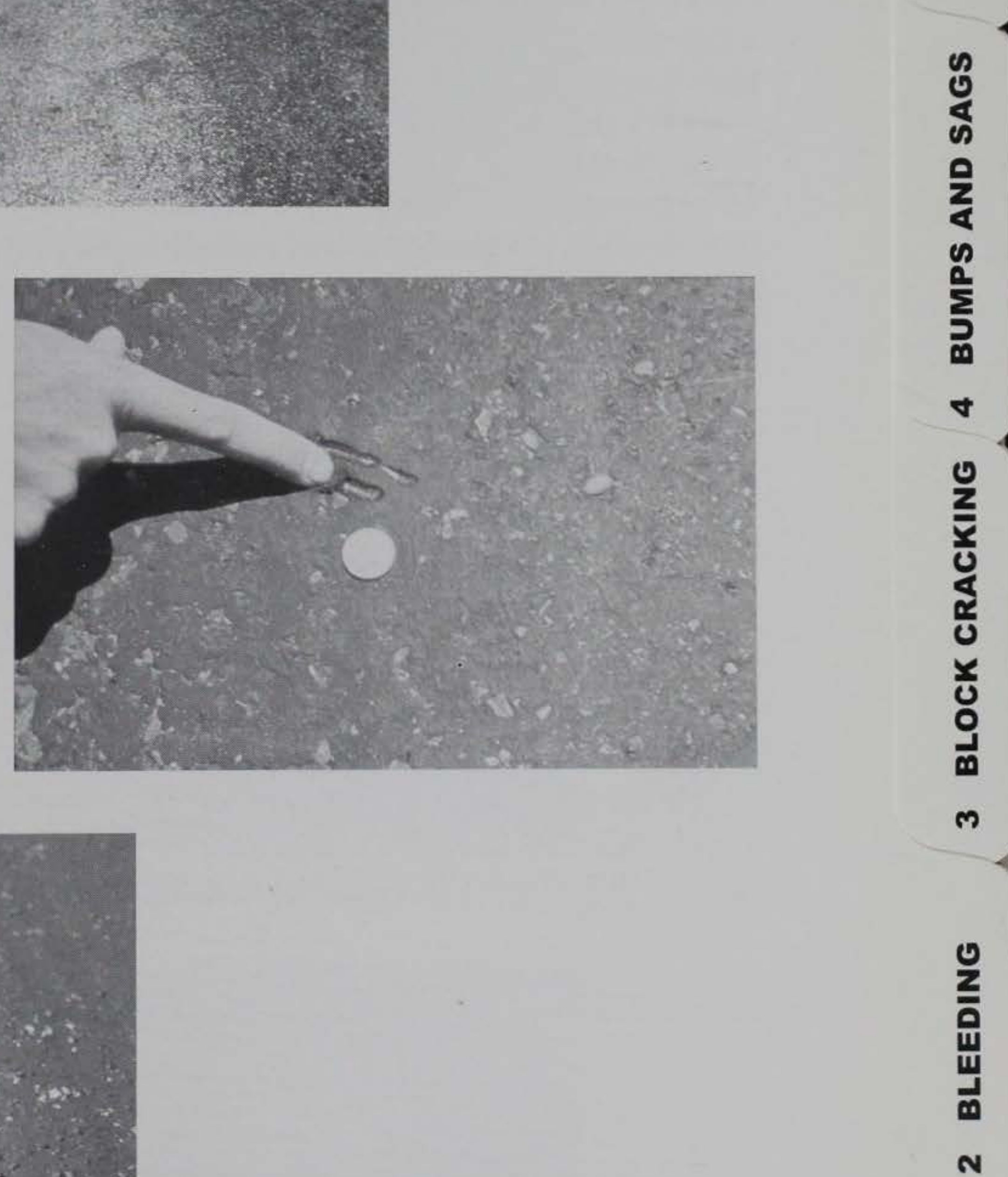

Figure 2c. High-Severity Bleeding.

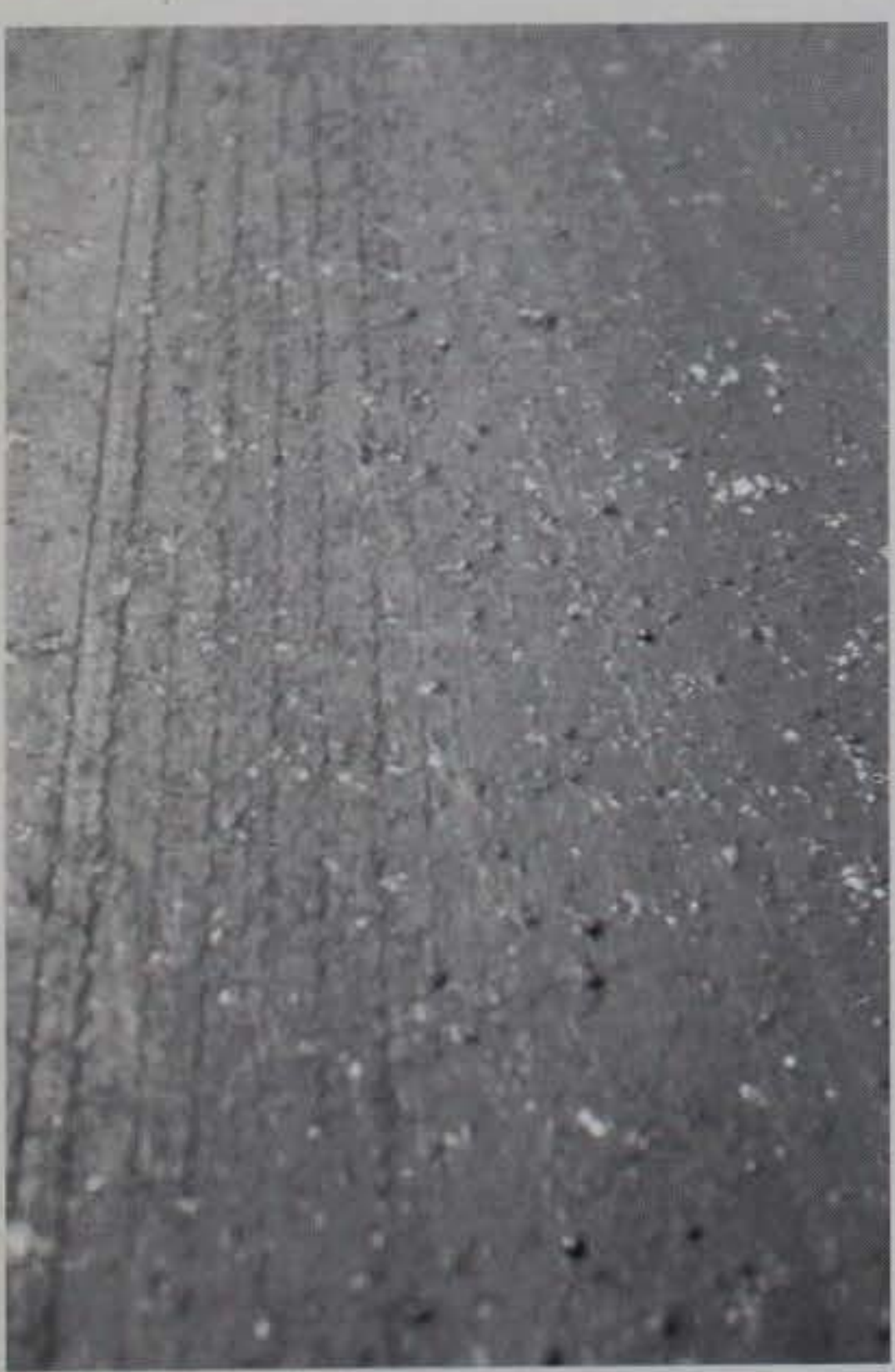

음 


\section{Block Cracking}

\section{Description}

Block cracks are interconnected cracks that divide the pavement into approximately rectangular pieces. The blocks may range in size from approximately 0.3 by $0.3 \mathrm{~m}$ ( 1 by $1 \mathrm{ft}$ ) to 3 by $3 \mathrm{~m}$ ( 10 by $10 \mathrm{ft}$ ). Block cracking is caused mainly by shrinkage of the asphalt concrete and daily temperature cycling (which results in daily stress/strain cycling). It is not load-associated. Block cracking usually indicates that the asphalt has hardened significantly. Block cracking normally occurs over a large portion of the pavement area, but sometimes will occur only in non-traffic areas. This type of distress differs from alligator cracking in that alligator cracks form smaller, many-sided pieces with sharp angles. Also, unlike block, alligator cracks are caused by repeated traffic loadings, and are therefore found only in traffic areas (i.e., wheel paths).

\section{Severity Levels}

L Blocks are defined by low-severity* cracks (Figure $3 a$ ).

M Blocks are defined by medium-severity ${ }^{\star}$ cracks (Figure $3 b$ ).

H Blocks are defined by high-severity* cracks (Figure $3 c$ ).

\section{How to Measure}

Block cracking is measured in square meters (feet) of surface area. It usually occurs at one severity level in a given pavement section. However, if areas of different severity levels can be easily distinguished from one another, they should be measured and recorded separately.

*See definitions of longitudinal transverse cracking. 


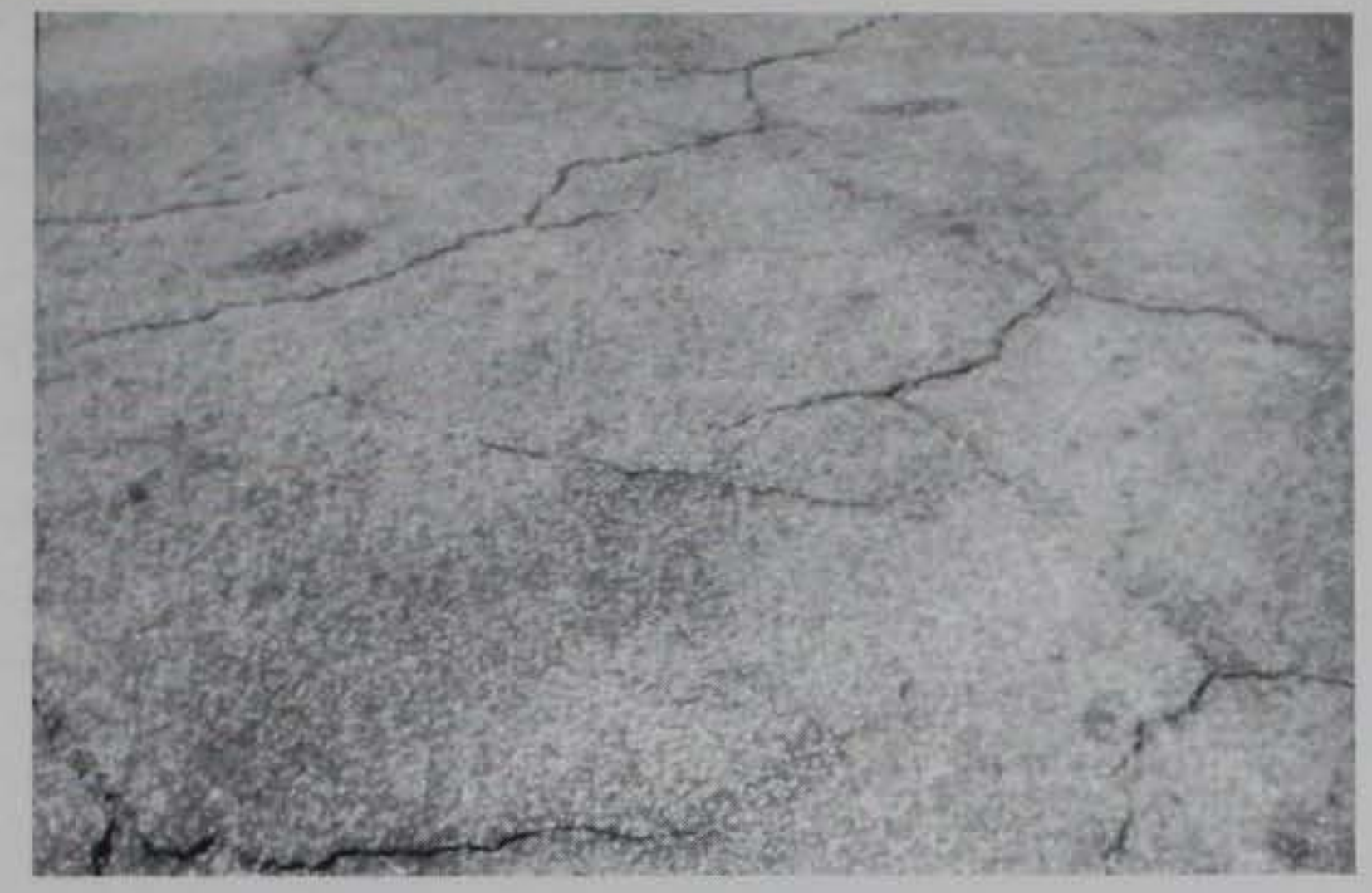

Figure 3a. Low-Severity Block Cracking.

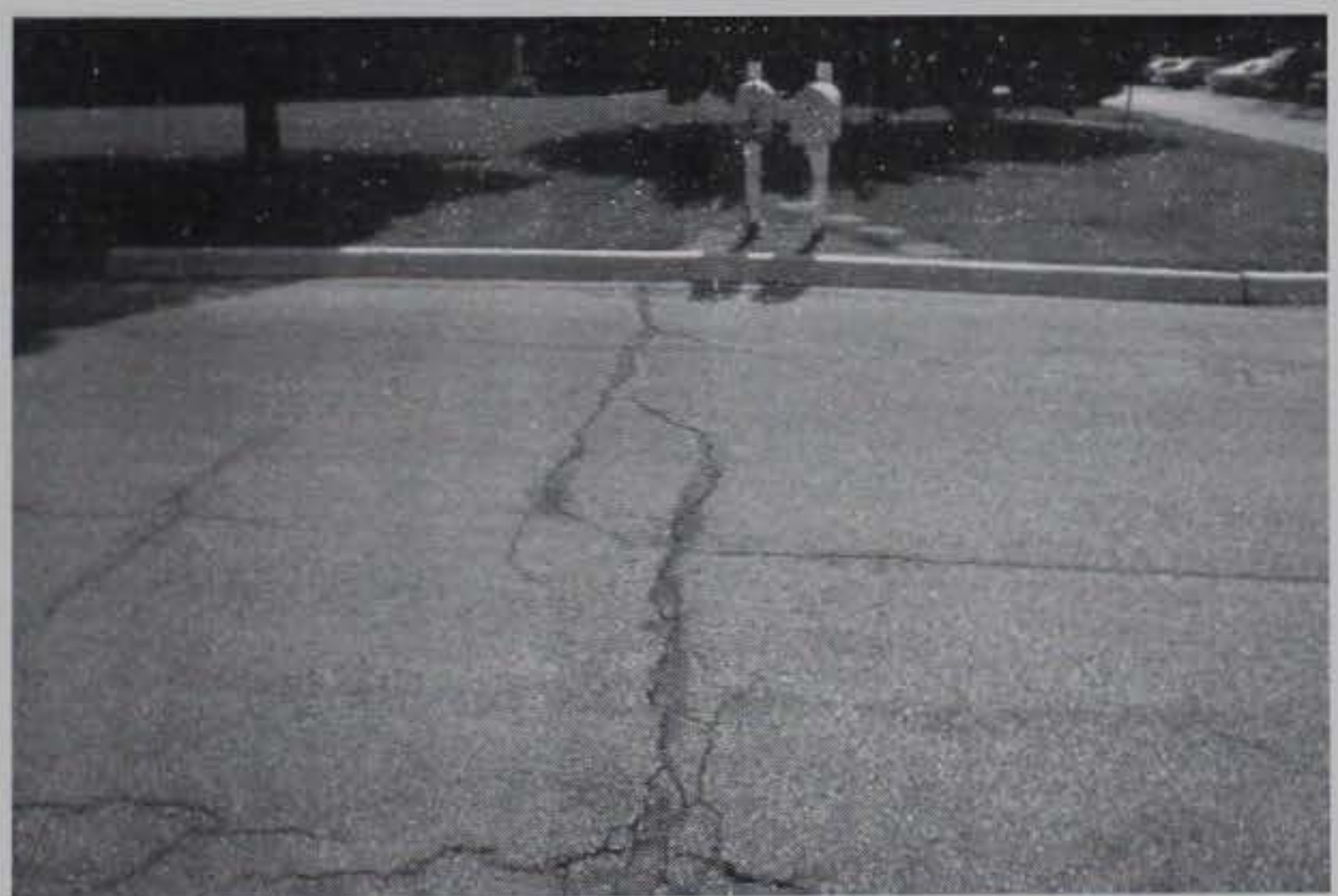

Figure 3b. Medium-Severity Block Cracking.

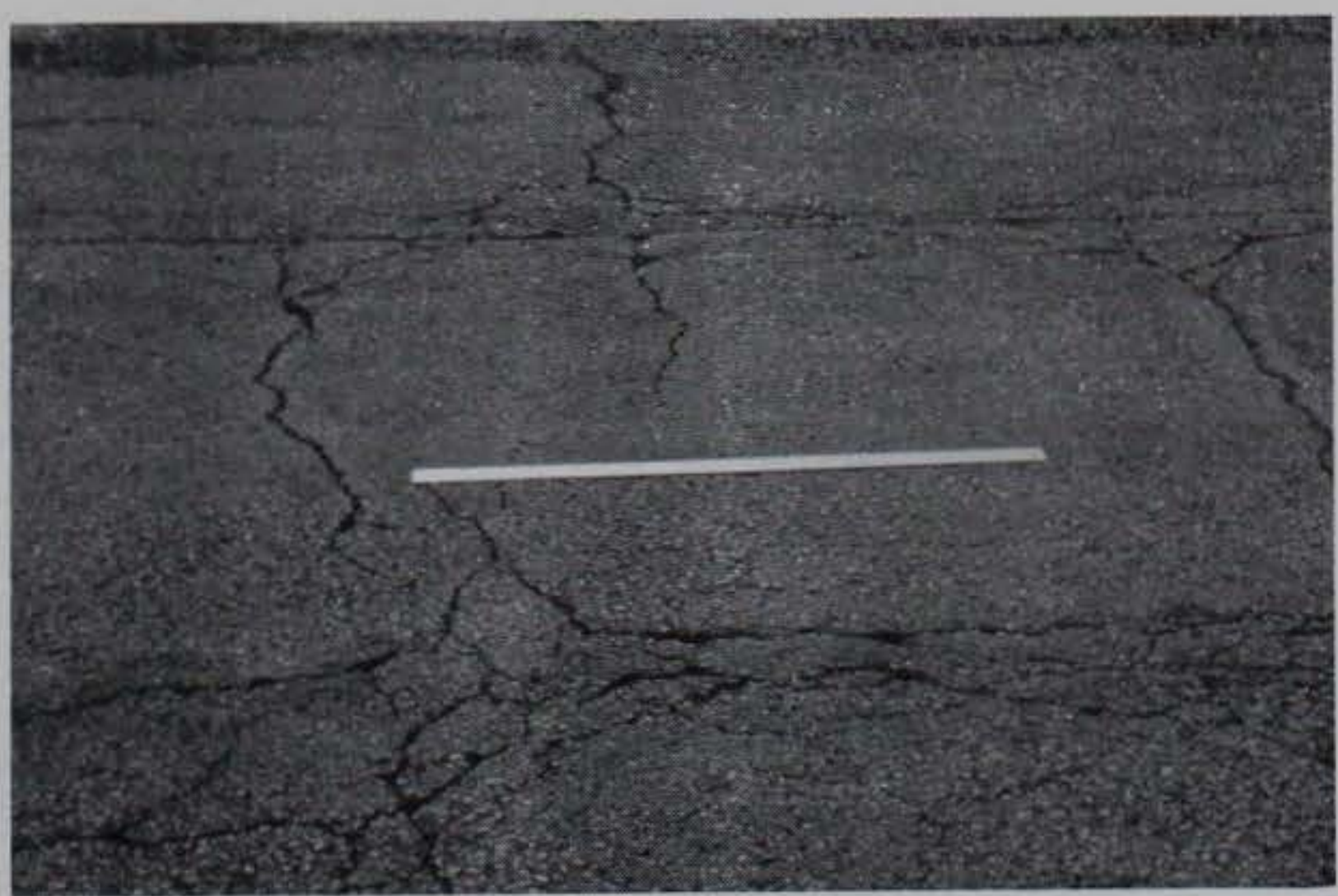

Figure 3c. High-Severity Block Cracking. 


\section{Bumps and Sags}

\section{Description}

Bumps are small, localized, upward displacements of the pavement surface. They are different from shoves in that shoves are caused by unstable pavement. Bumps, on the other hand, can be caused by several factors, including:

1. Buckling or bulging of underlying PCC slabs in AC overlay over PCC pavement.

2. Frost heave (ice, lens growth).

3. Infiltration and buildup of material in a crack in combination with traffic loading (sometimes called "tenting").

Sags are small, abrupt, downward displacements of the pavement surface. If bumps appear in a pattern perpendicular to traffic flow and are spaced at less than $3 \mathrm{~m}(10 \mathrm{ft})$, the distress is called corrugation. Distortion and displacement that occur over large areas of the pavement surface, causing large and/or long dips in the pavement should be recorded as "swelling."

\section{Severity Levels}

L Bump or sag causes low-severity ride quality (Figure 4a).

M Bump or sag causes medium-severity ride quality (Figure 4b).

H Bump or sag causes high-severity ride quality (Figure 4c).

\section{How to Measure}

Bumps or sags are measured in linear meters (feet). If the bump occurs in combination with a crack, the crack is also recorded. 


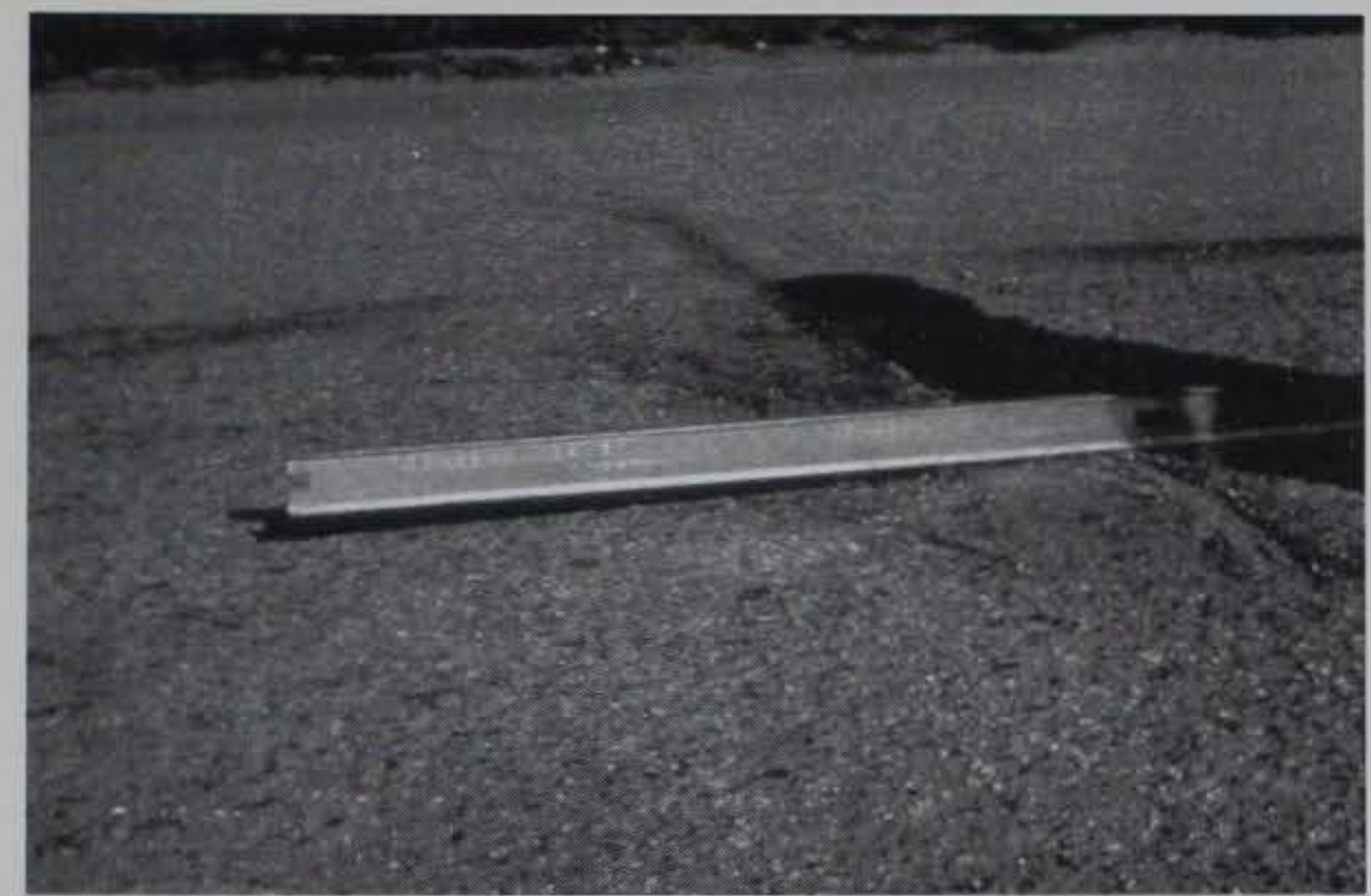

Figure 4a. Low-Severity Bumps and Sags.

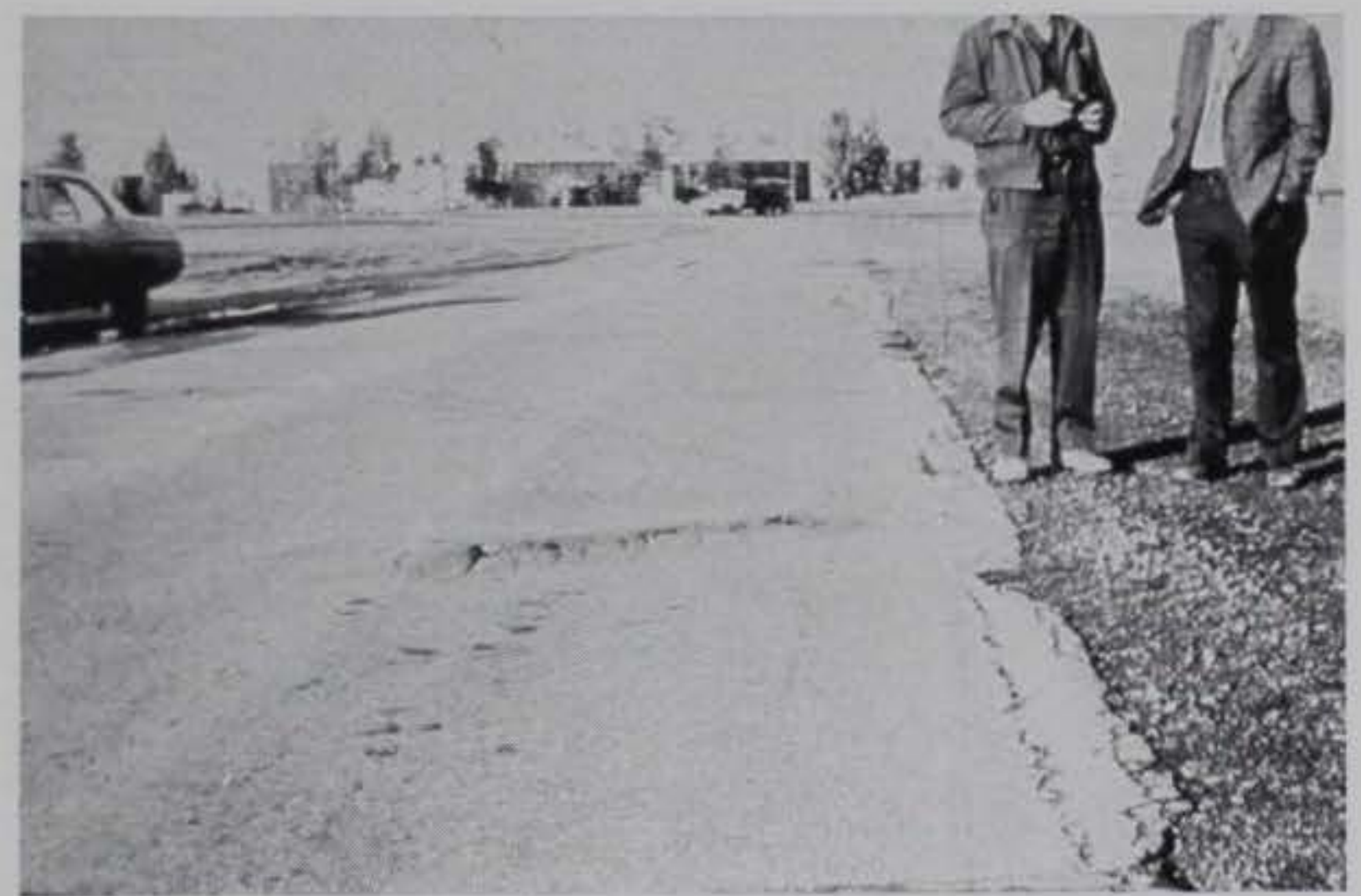

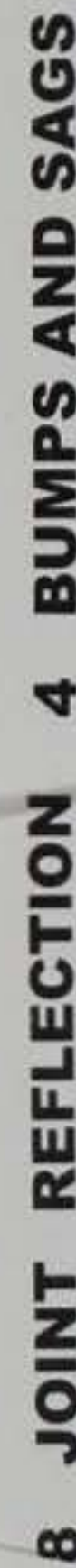

Figure $4 b$. Medium-Severity Bumps and Sags.

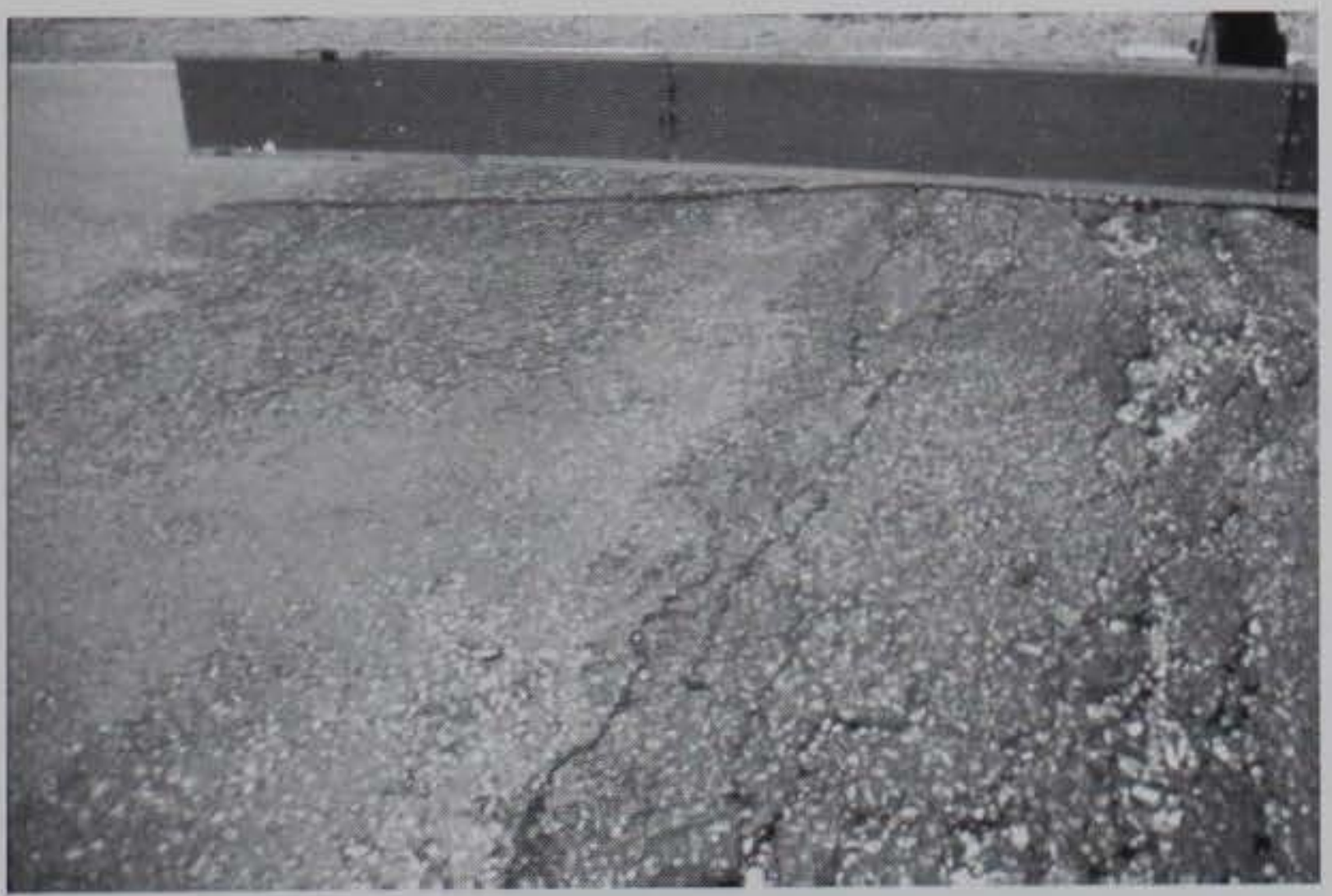

Figure 4c. High-Severity Bumps and Sags. 


\section{Corrugation}

\section{Description}

Corrugation (also known as "washboarding") is a series of closely spaced ridges and valleys (ripples) occurring at fairly regular intervals, usually less than $3 \mathrm{~m}(10 \mathrm{ft})$ along the pavement. The ridges are perpendicular to the traffic direction. This type of distress is usually caused by traffic action combined with an unstable pavement surface or base.

\section{Severity Levels}

L Corrugation produces low-severity ride quality (Figure 5a).

M Corrugation produces medium-severity ride quality (Figure $5 b$ ).

H Corrugation produces high-severity ride quality (Figure 5c).

\section{How to Measure}

Corrugation is measured in square meters (feet) of surface area. 


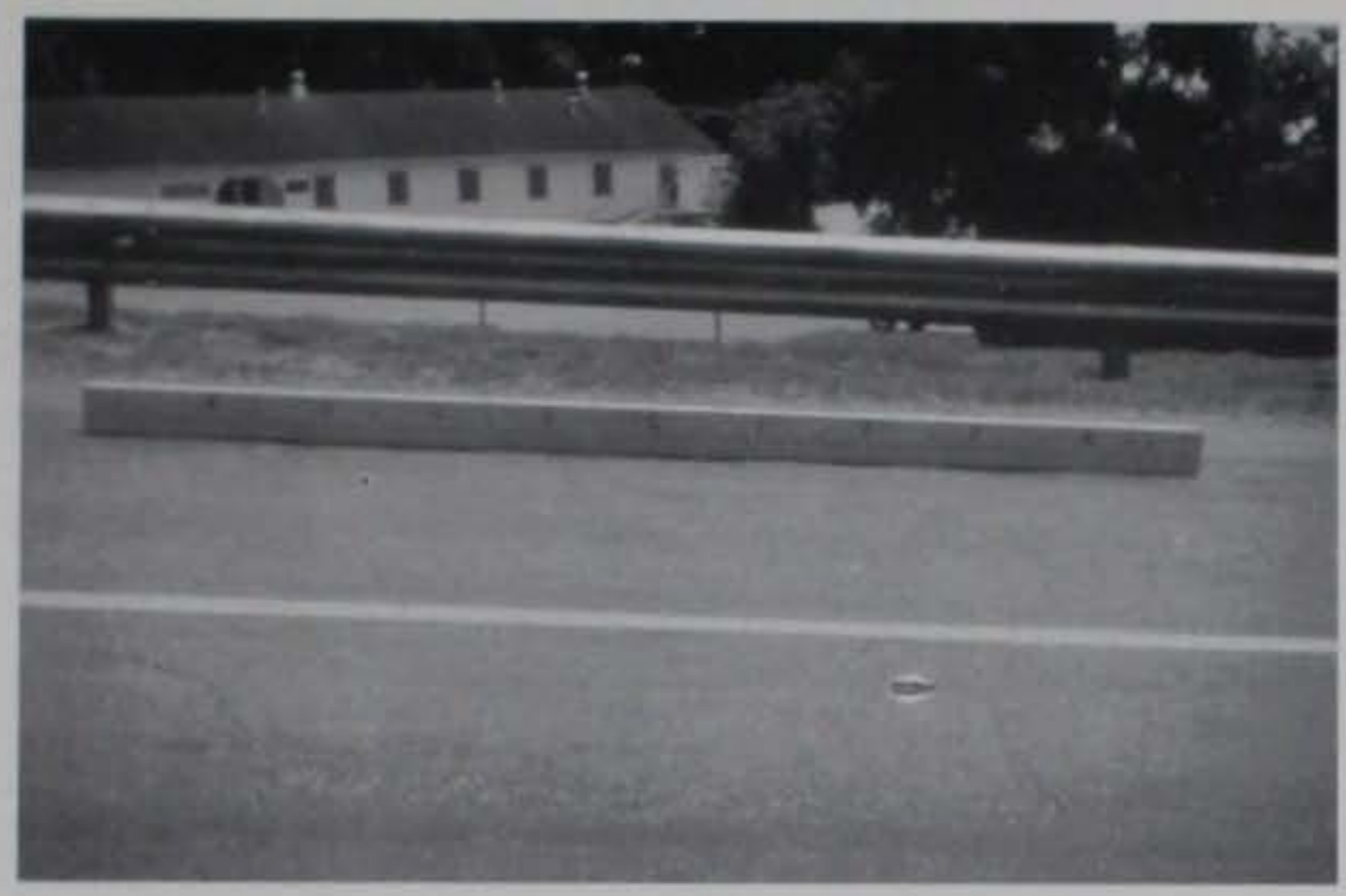

Figure 5a. Low-Severity Corrugation.

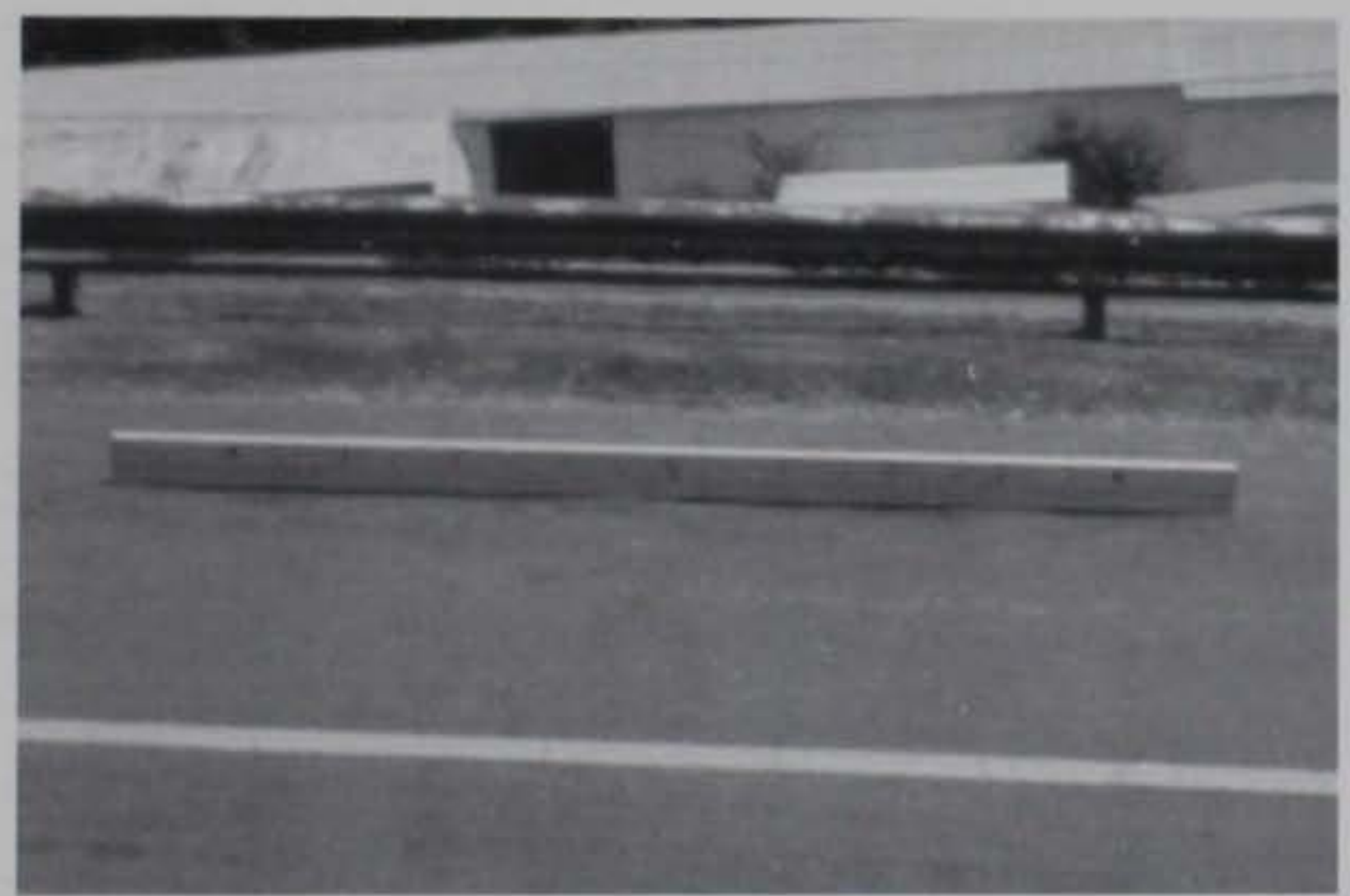

Figure 5b. Medium-Severity Corrugation.

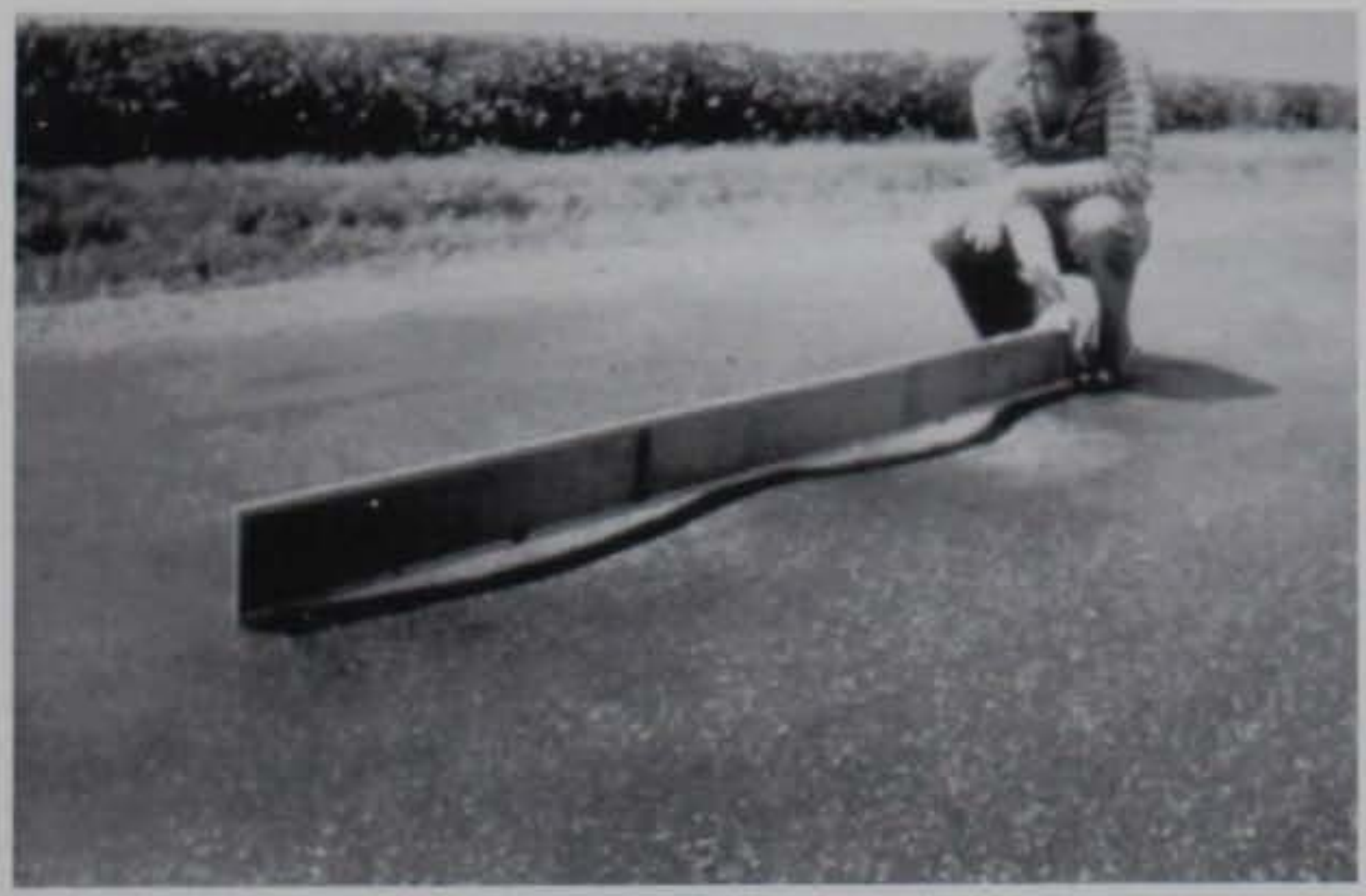

Figure 5c. High-Severity Corrugation. 


\section{Depression}

\section{Description}

Depressions are localized pavement surface areas with elevations slightly lower than those of the surrounding pavement. In many instances, light depressions are not noticeable until after a rain, when ponding water creates a "birdbath" area; on dry pavement, depressions can be spotted by looking for stains caused by ponding water. Depressions are created by settlement of the foundation soil or are a result of improper construction. Depressions cause some roughness, and when deep enough or filled with water, can cause hydroplaning.

\section{Severity Levels}

Maximum Depth of Depression

L 13 to $25 \mathrm{~mm}(1 / 2$ to $1 \mathrm{in}$.) (Figure $6 \mathrm{a})$.

M 25 to $50 \mathrm{~mm}$ (1 to 2 in.) (Figure $6 \mathrm{~b}$ ).

H more than $50 \mathrm{~mm}$ (2 in.) (Figure 6c).

\section{How to Measure}

Depressions are measured in square meters (feet) of surface area. 


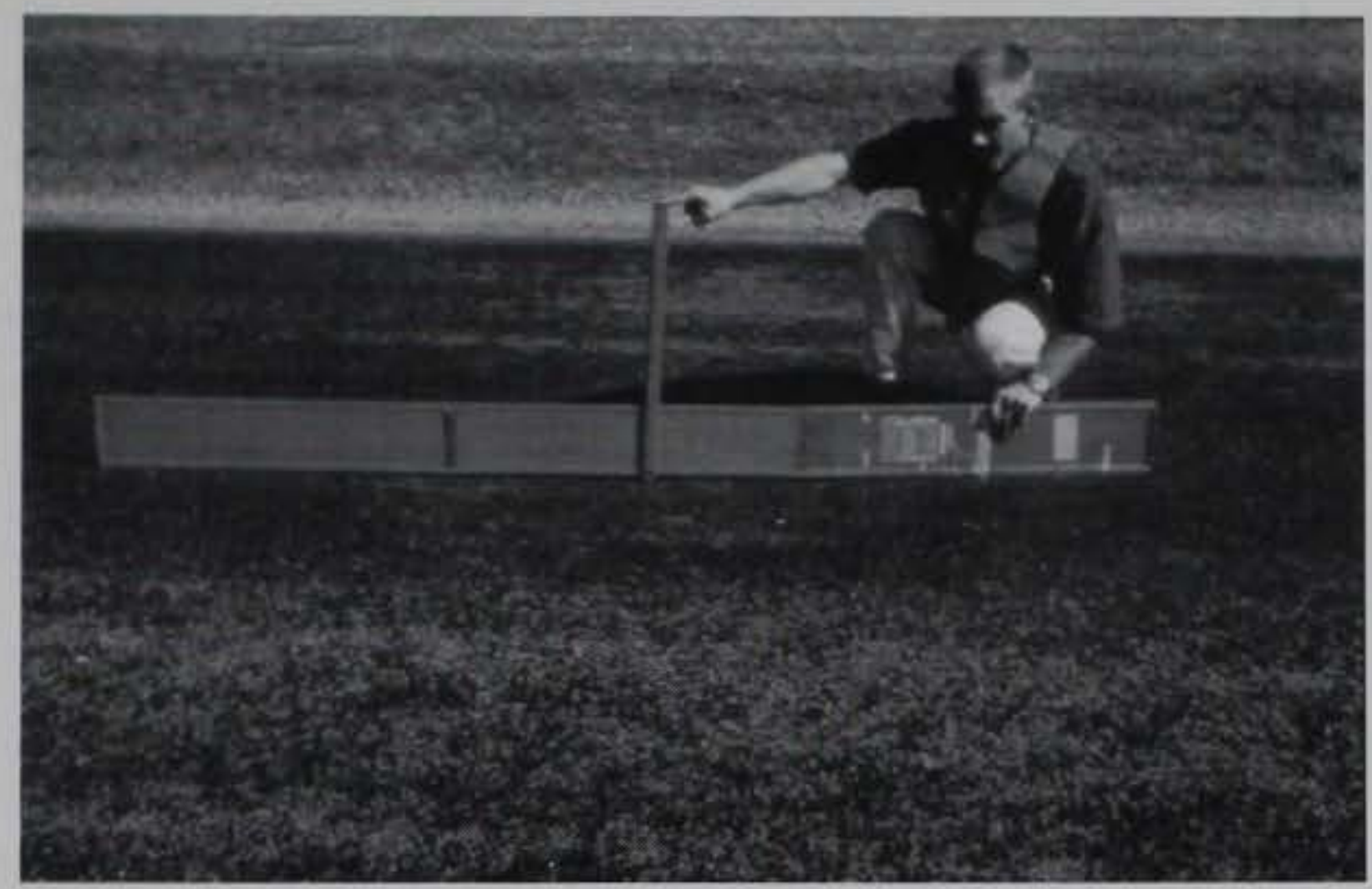

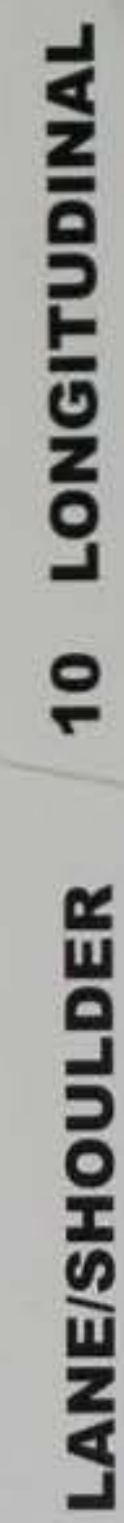

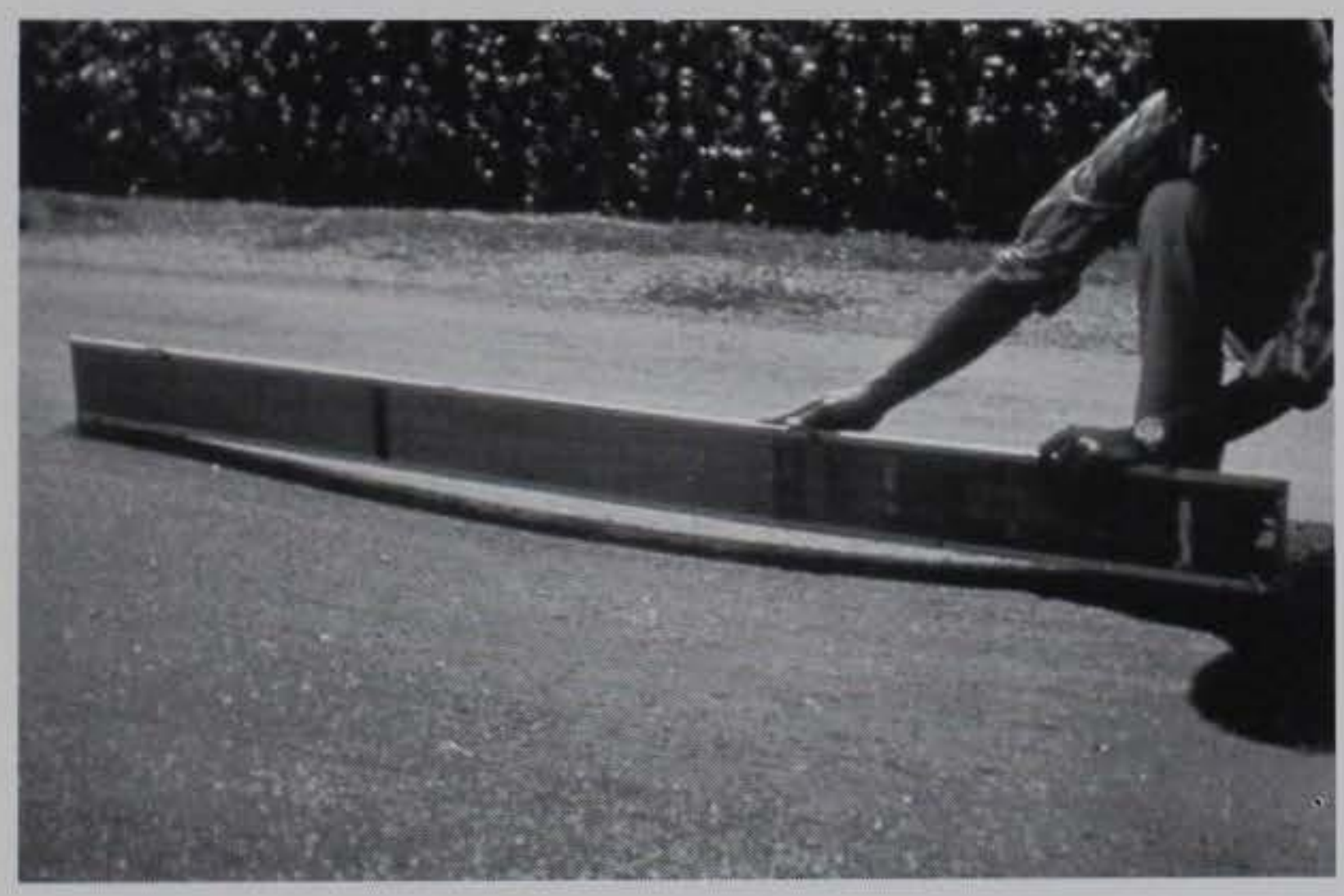

の

Figure 6b. Medium-Severity Depression.

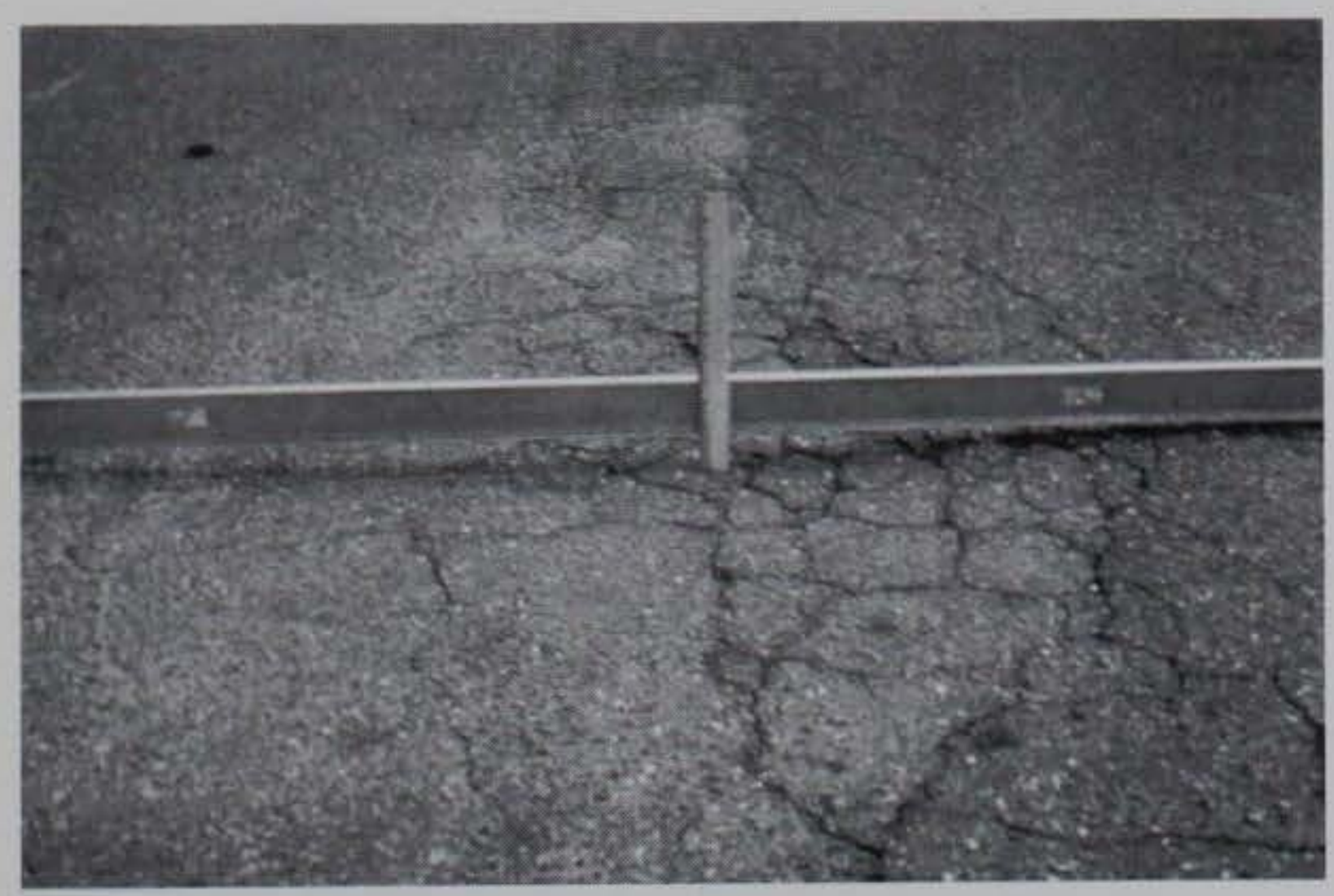

Figure 6c. High-Severity Depression. 


\section{Edge Cracking}

\section{Description}

Edge cracks are parallel to and usually within 0.3 to $0.5 \mathrm{~m}$ ( 1 to $1.5 \mathrm{ft}$ ) of the outer edge of the pavement. This distress is accelerated by traffic loading and can be caused by frost-weakened base or subgrade near the edge of the pavement. The area between the crack and pavement edge is classified as raveled if it is broken up (sometimes to the extent that pieces are removed).

\section{Severity Levels}

L Low or medium cracking with no breakup or raveling (Figure 7a).

M Medium cracks with some breakup and raveling (Figure 7b).

H Considerable breakup or raveling along the edge (Figure 7c).

\section{How to Measure}

Edge cracking is measure in linear meters (feet). 


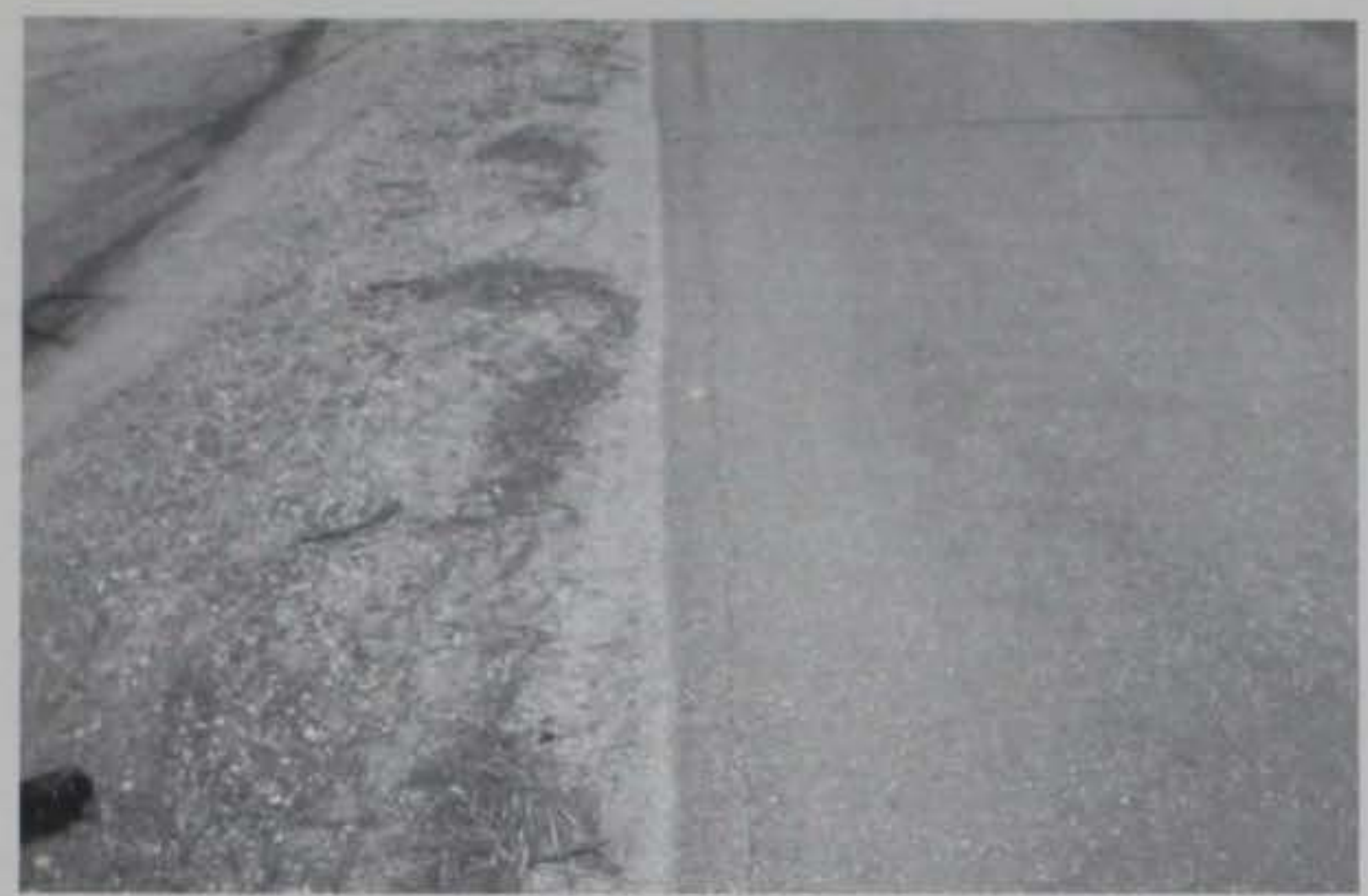

Figure 7a. Low-Severity Edge Cracking.

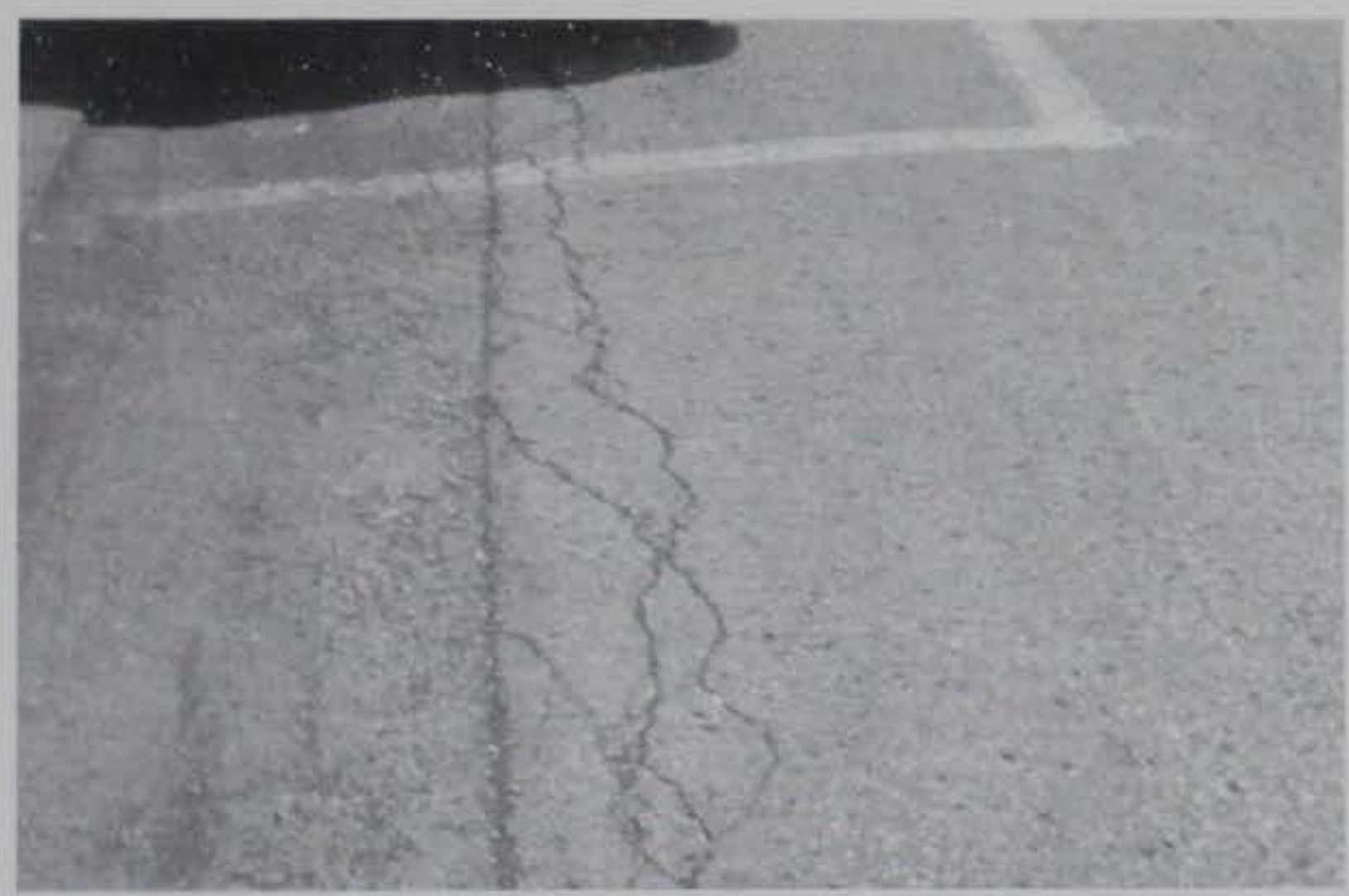

Figure 7b. Medium-Severity Edge Cracking.

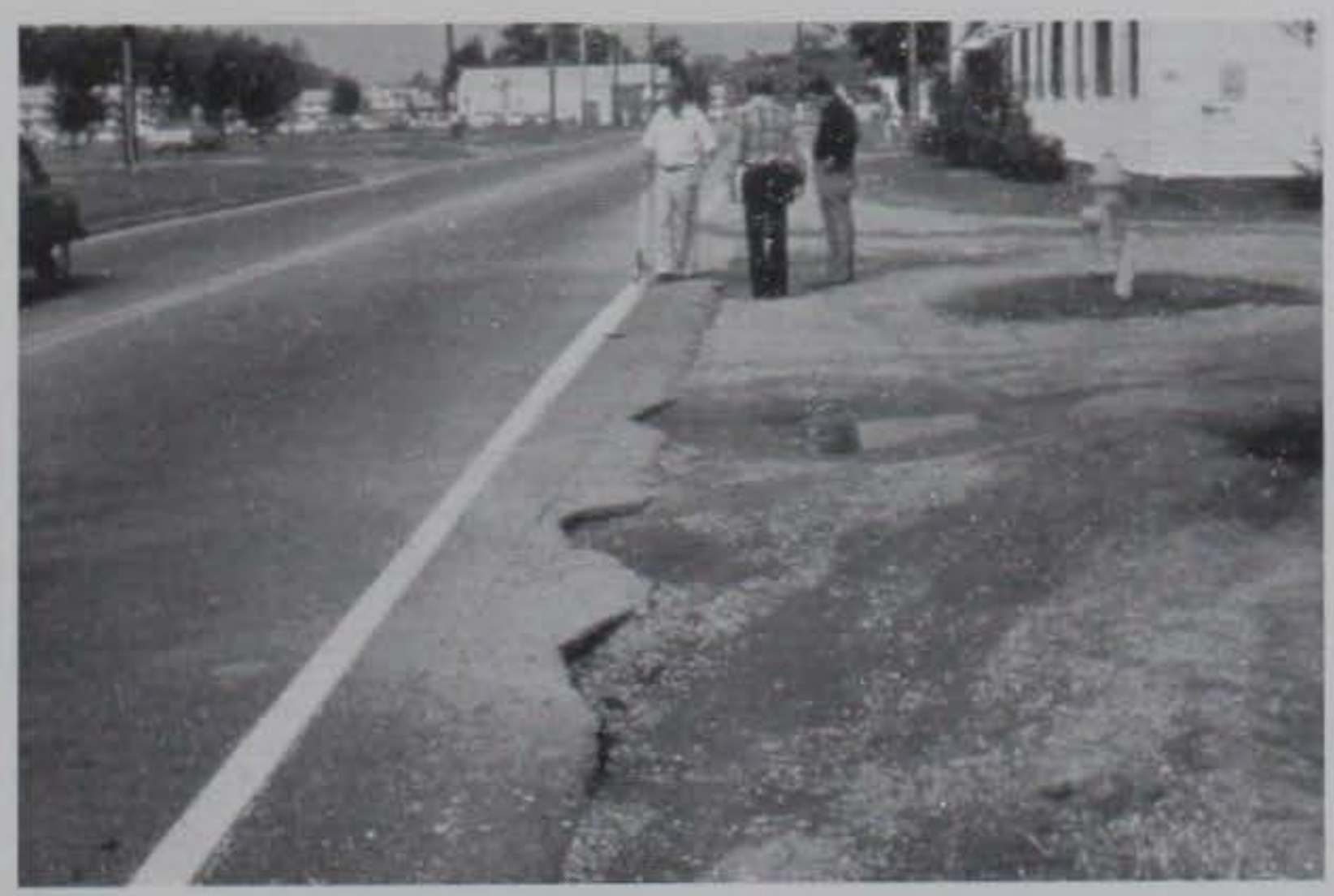

Figure 7c. High-Severity Edge Cracking. 


\section{Joint Reflection Cracking \\ (From Longitudinal and Transverse PCC Slabs)}

\section{Description}

This distress occurs only on asphalt-surfaced pavements that have been laid over a PCC slab. It does not include reflection cracks from any other type of base (i.e., cement- or lime-stabilized); these cracks are caused mainly by thermal- or moisture-induced movement of the PCC slab beneath the AC surface. This distress is not load-related; however, traffic loading may cause a breakdown of the AC surface near the crack. If the pavement is fragmented along a crack, the crack is said to be spalled. A knowledge of slab dimension beneath the AC surface will help to identify these distresses.

\section{Severity Levels}

L One of the following conditions exists (Figure 8a): (1) Non-filled crack width is less than $10 \mathrm{~mm}$ ( $3 / 8 \mathrm{in}$.), or (2) filled crack of any width (filler in satisfactory condition).

M One of the following conditions exists (Figure $8 b$ ): (1) Non-filled crack width is greater than or equal to $10 \mathrm{~mm}(3 / 8 \mathrm{in}$.) and less than $75 \mathrm{~mm}$ ( $3 \mathrm{in}$.); ( 2 ) non-filled crack less than or equal to 75 $\mathrm{mm}$ ( $3 \mathrm{in}$.) surrounded by light secondary cracking, or (3) filled crack of any width surrounded by light secondary cracking.

H One of the following conditions exists (Figure 8c): (1) Any crack filled or non-filled surrounded by medium or high severity secondary cracking; (2) non-filled cracks greater than $75 \mathrm{~mm}$ (3 in.), or (3) A crack of any width where approximately $100 \mathrm{~mm}$ (4 in.) of pavement around the crack are severely raveled or broken.

\section{How to Measure}

Joint reflection cracking is measured in linear meters (feet). The length and severity level of each crack should be identified and recorded separately. For example, a crack that is $15 \mathrm{~m}(50 \mathrm{ft})$ long may have $3 \mathrm{~m}(10 \mathrm{ft})$ of high severity cracks; these are all recorded separately. If a bump occurs at the reflection crack, it is also recorded. 
Figure 8a. Low-Severity

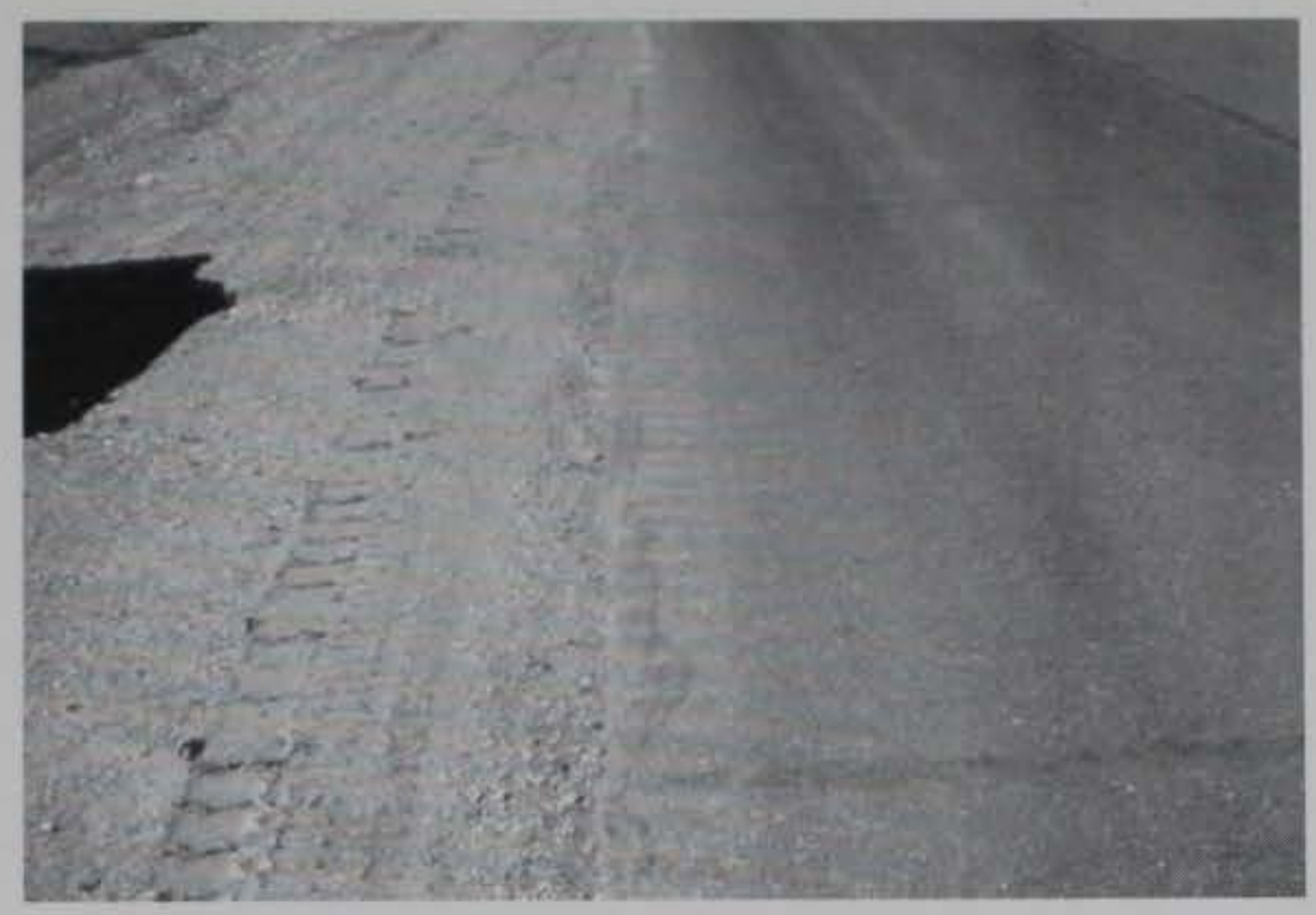
Joint Reflection Cracking.

Figure $8 b$. Medium-Severity Joint Reflection Cracking.
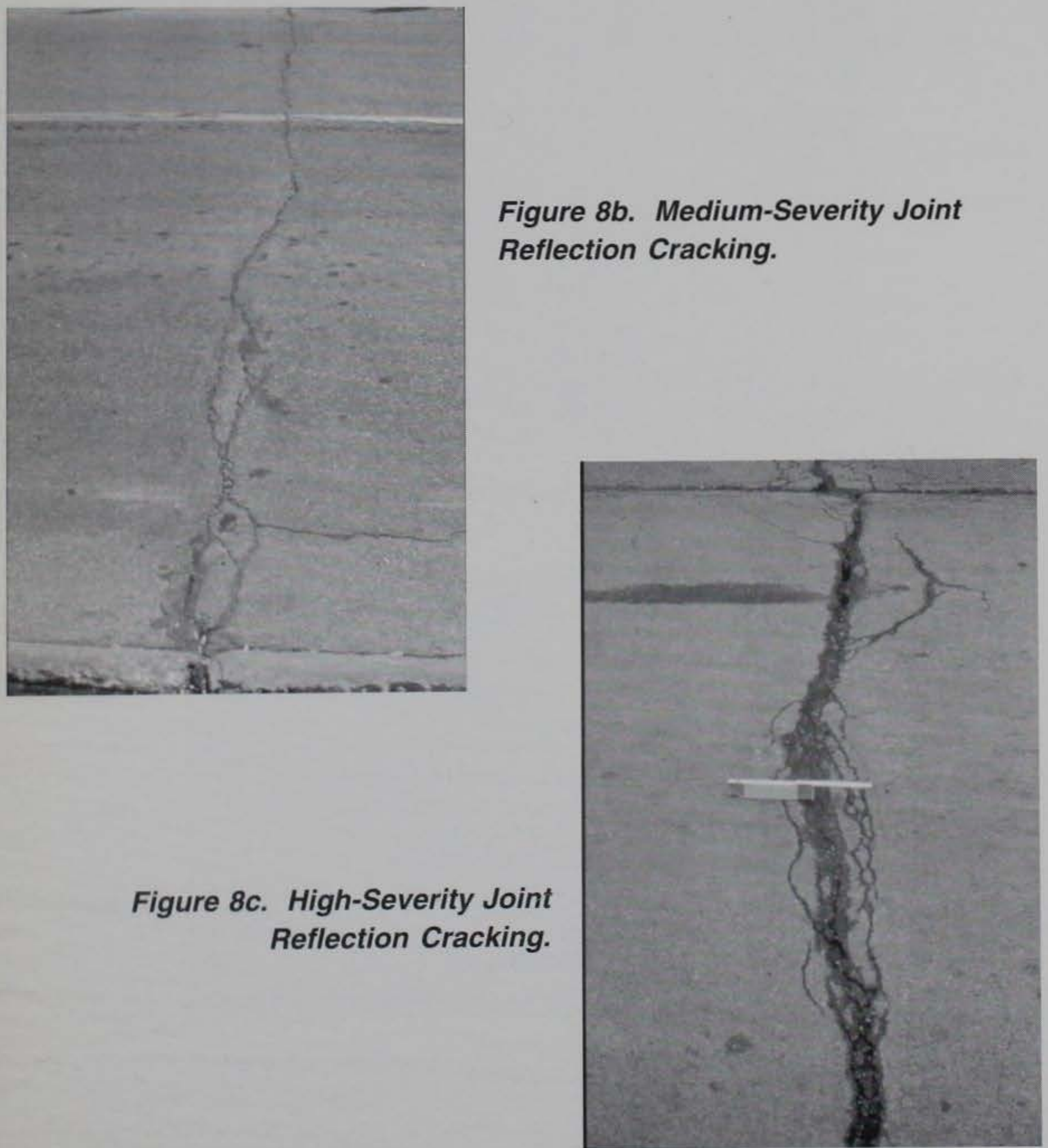

$\infty$

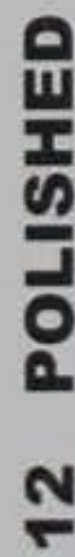

Figure 8c. High-Severity Joint Reflection Cracking. 


\section{Lane/Shoulder Drop-Off}

\section{Description}

Lane/shoulder drop-off is a difference in elevation between the pavement edge and the shoulder. This distress is caused by shoulder erosion, shoulder settlement, or by building up the roadway without adjusting the shoulder level.

\section{Severity Levels}

L The difference in elevation between the pavement edge and shoulder is $>25 \mathrm{~mm}$ ( $1 \mathrm{in}$.) and $<50 \mathrm{~mm}$ ( $2 \mathrm{in}$.). (Figure 9a).

M The difference in elevation is $>50 \mathrm{~mm}$ (2 in.) and $<100 \mathrm{~mm}$ (4 in.). (Figure 9b).

H The difference in elevation is $>100 \mathrm{~mm}$ (4 in.). (Figure 9c).

\section{How to Measure}

Lane/shoulder drop-off is measured in linear meters (feet). 


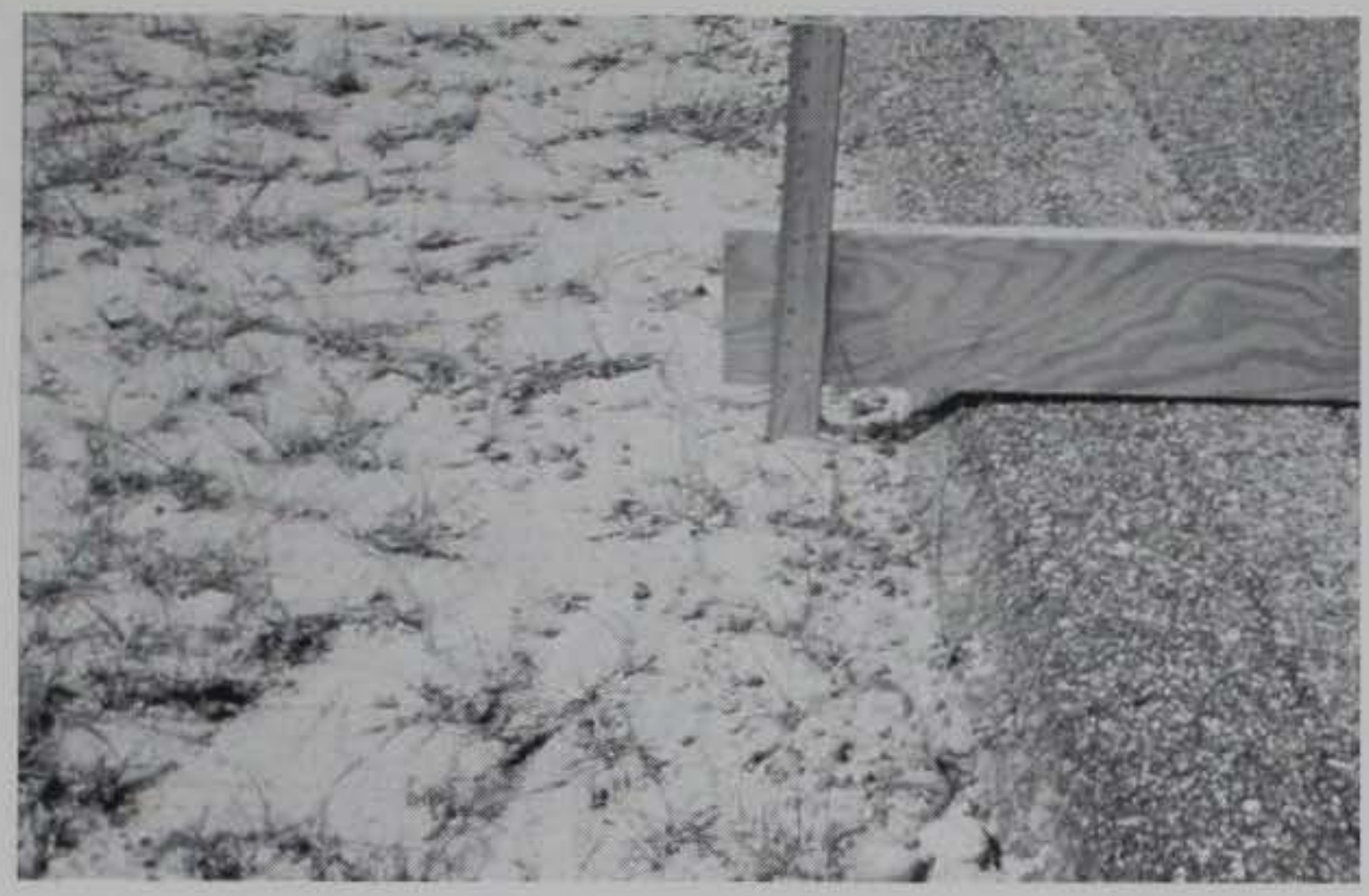

Figure 9a. Low-Severity Lane/Shoulder Drop-Off.

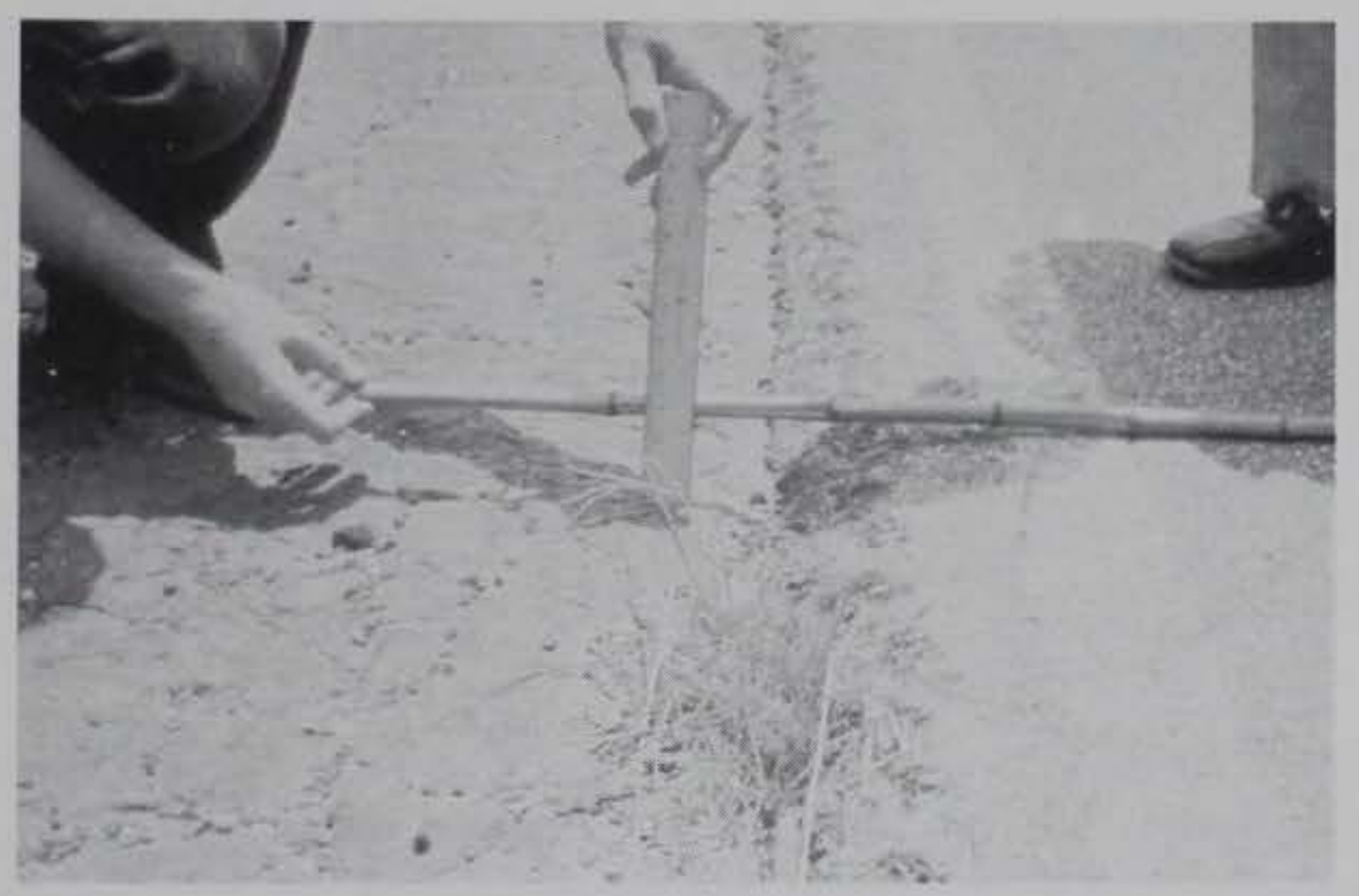

の

Figure 9b. Medium-Severity Lane/Shoulder Drop-Off.

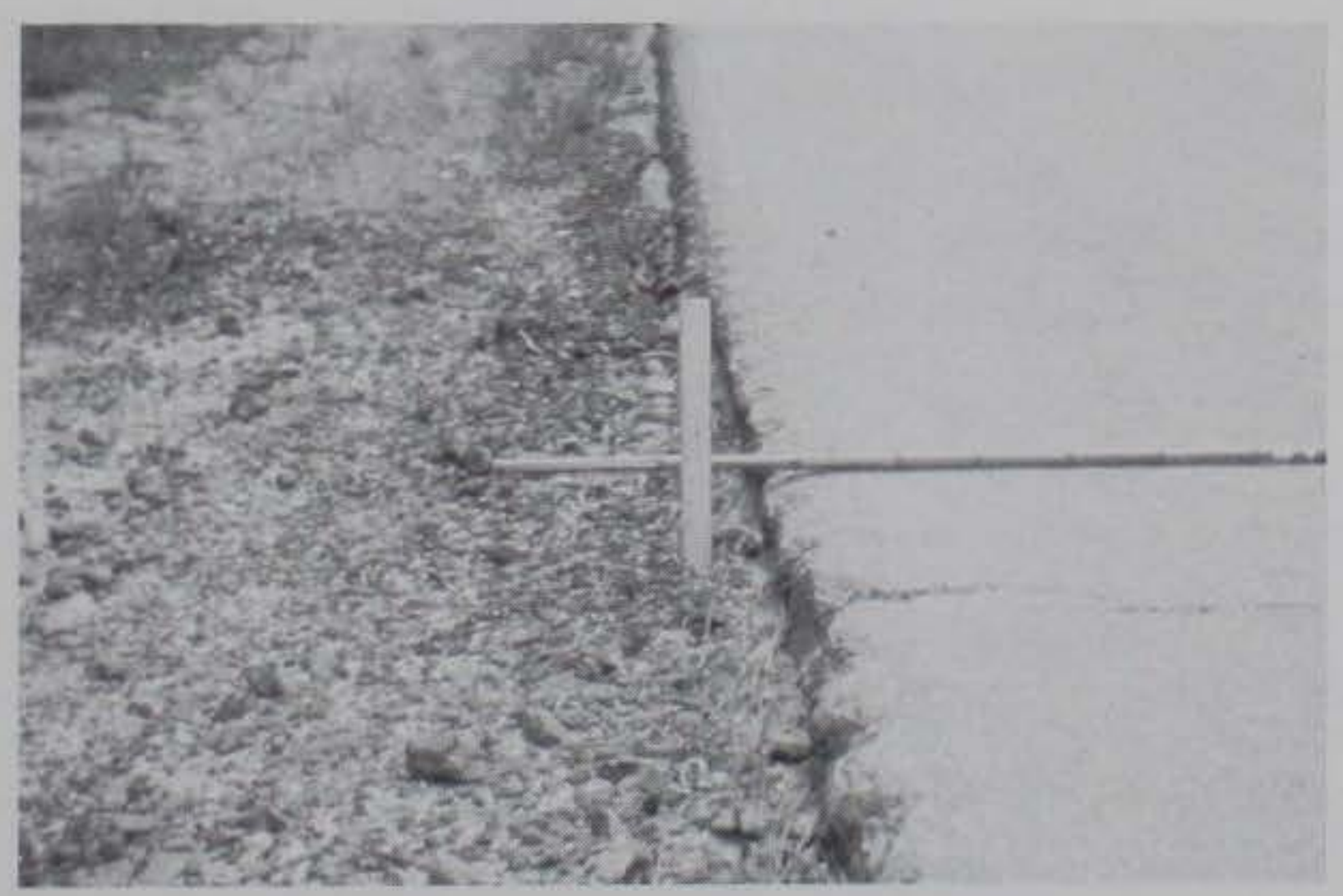

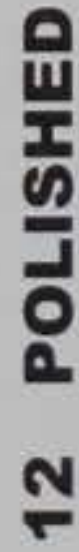

Figure 9c. High-Severity Lane/Shoulder Drop-Off. 


\section{Longitudinal and Transverse Cracking (Non-PCC Slab Joint Reflective)}

\section{Description}

Longitudinal cracks are parallel to the pavement's centerline or laydown direction. They may be caused by:

1. A poorly constructed paving lane joint.

2. Shrinkage of the AC surface due to low temperatures or hardening of the asphalt and/or daily temperature cycling.

3. A reflective crack caused by cracking beneath the surface course, including cracks in PCC slabs (but not PCC joints).

Transverse cracks extend across the pavement at approximately right angles to the pavement centerline or direction of laydown. These types of cracks are not usually load-associated.

\section{Severity Levels}

L One of the following conditions exists (Figure 10a): (1) non-filled crack width is less than $10 \mathrm{~mm}$ (3/8 in.), or (2) filled crack of any width (filler in satisfactory condition).

M One of the following conditions exists (Figure 10b): (1) non-filled crack width is greater than or equal to $10 \mathrm{~mm}$ and less than 75 $\mathrm{mm}$ ( $3 / 8$ to $3 \mathrm{in}$.); (2) non-filled crack is less than or equal to 75 $\mathrm{mm}$ ( 3 in.) surrounded by light and random cracking, or (3) filled crack is of any width surrounded by light random cracking.

H One of the following conditions exists (Figure 10c): (1) any crack filled or non-filled surrounded by medium- or high-severity random cracking, (2) non-filled crack greater than $75 \mathrm{~mm}$ (3 in.), or (3) a crack of any width where approximately $100 \mathrm{~mm}$ (4 in.) of pavement around the crack is severely broken.

\section{How to Measure}

Longitudinal and transverse cracks are measured in linear meters (feet). The length and severity of each crack should be recorded. If the crack does not have the same severity level along its entire length, each portion of the crack having a different severity level should be recorded separately. 


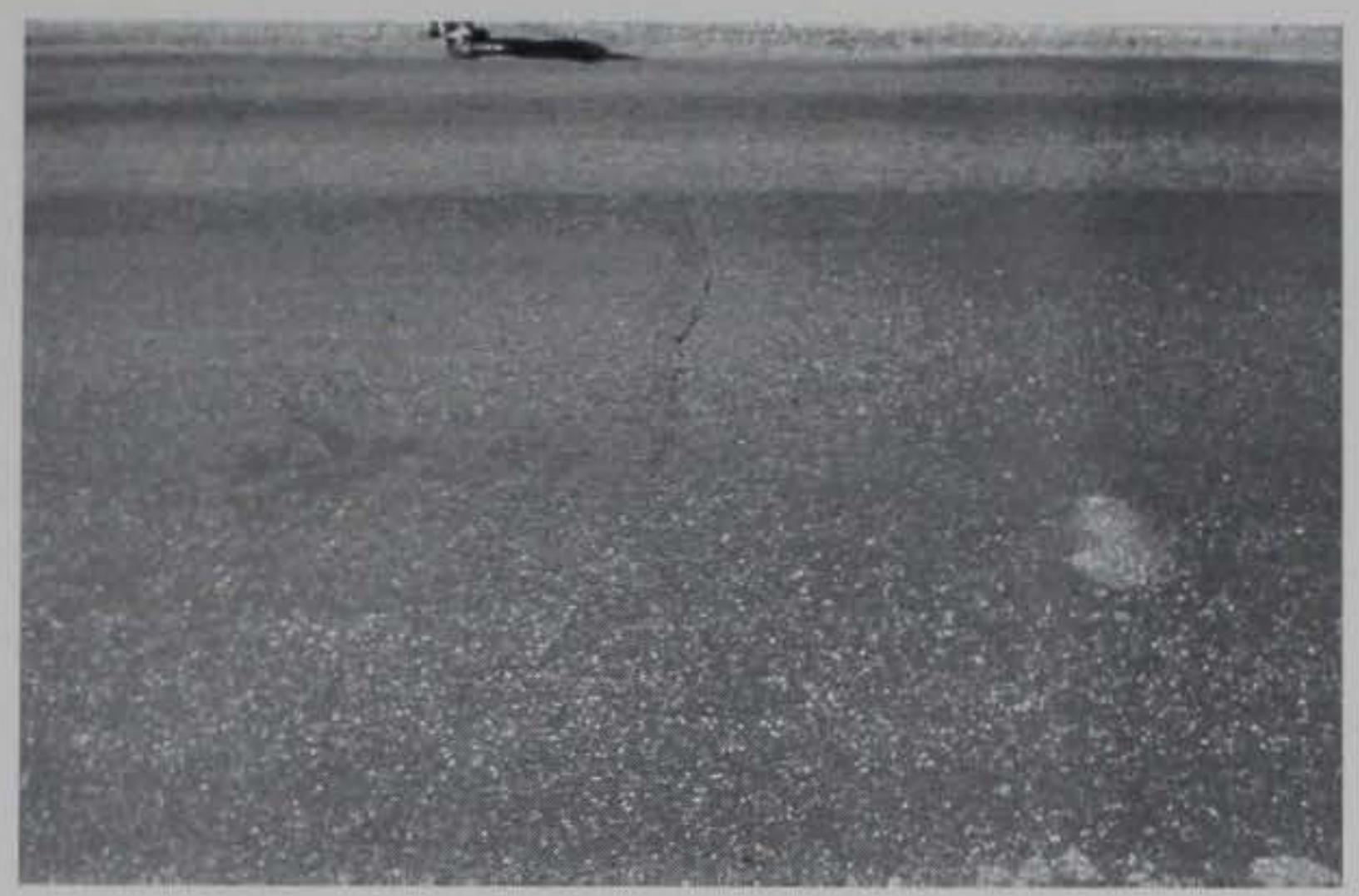

Figure 10a. Low-Severity Longitudinal and Transverse Cracking.

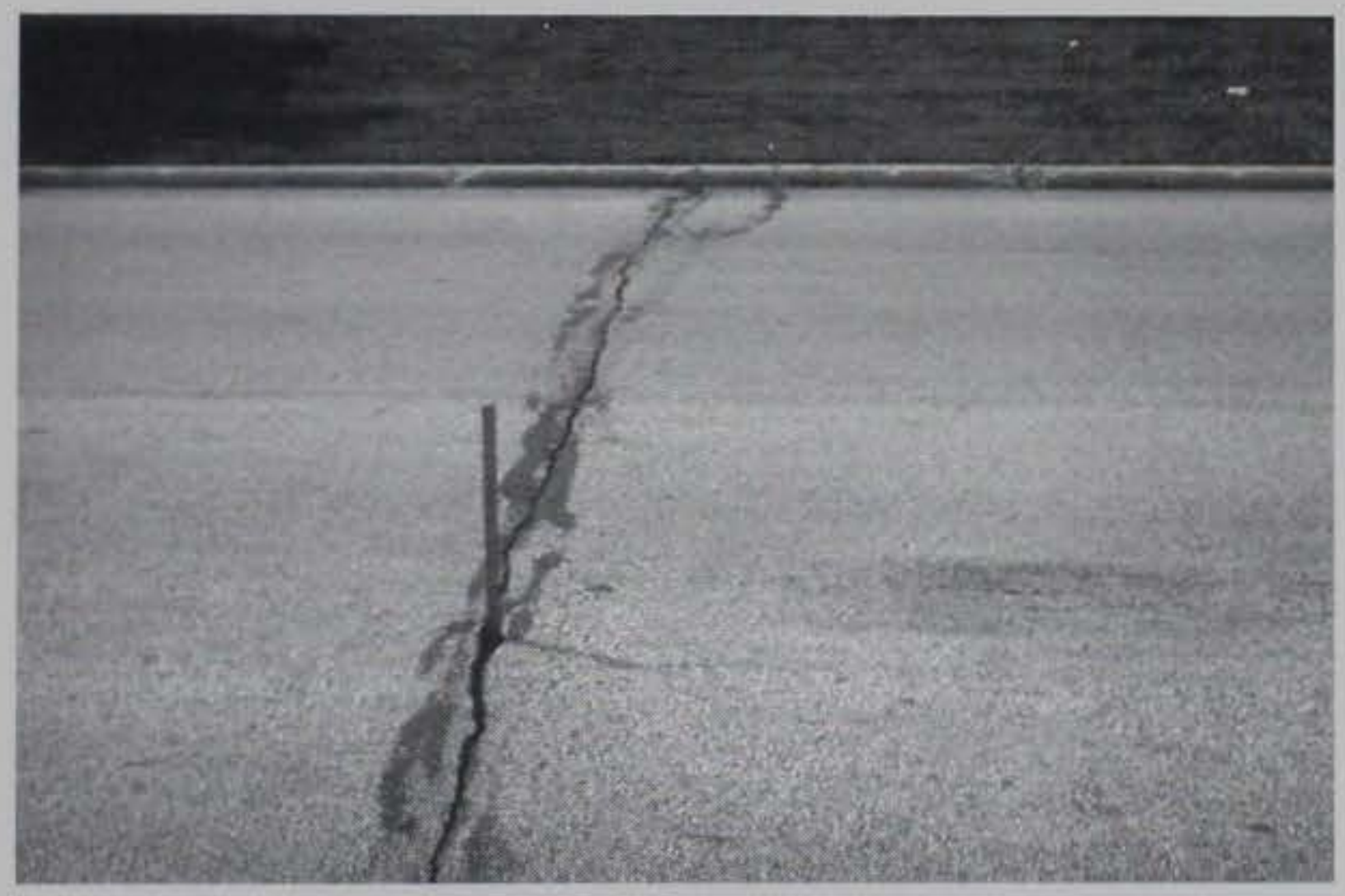

옹

Figure 10b. Medium-Severity Longitudinal and Transverse Cracking.

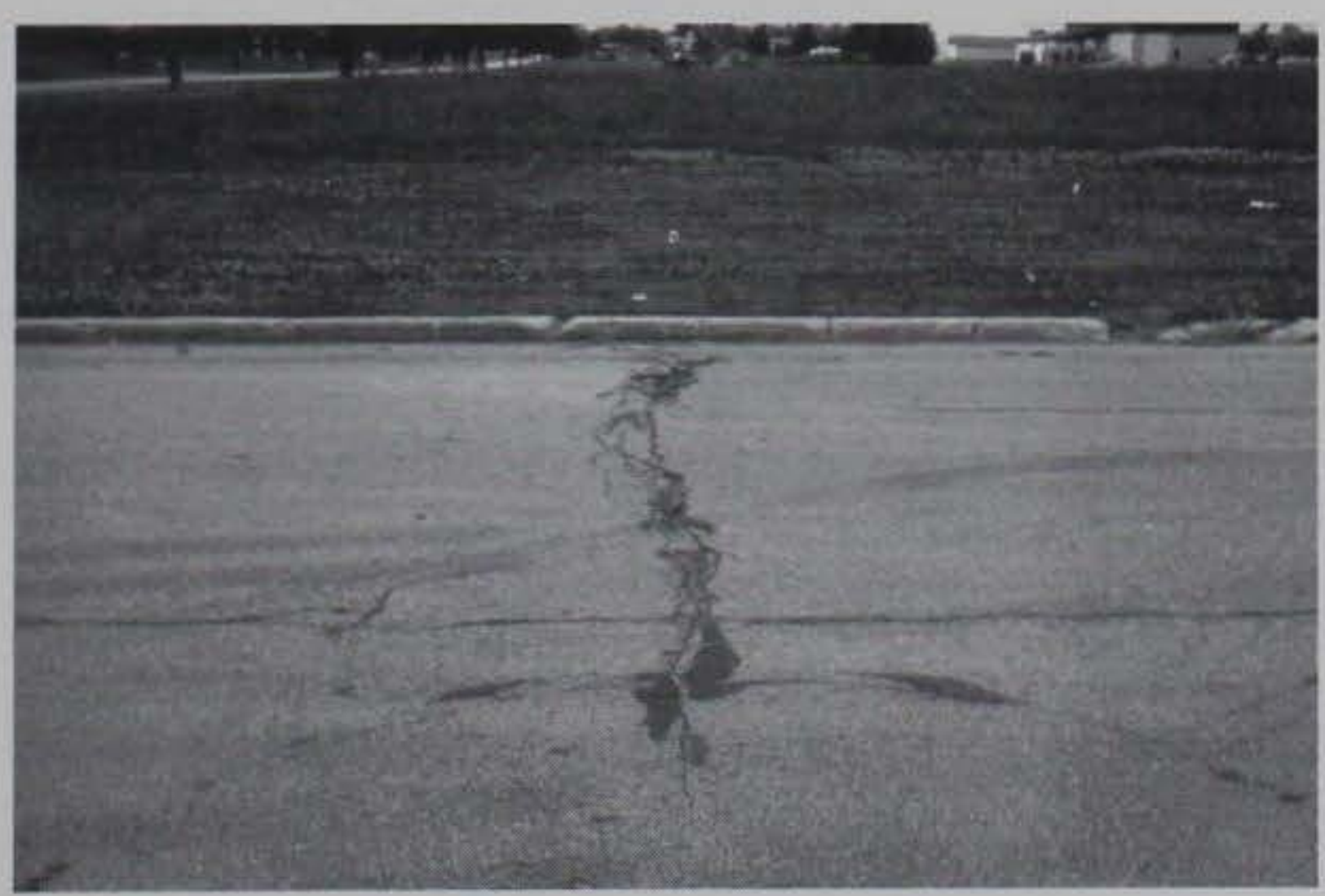

Figure 10c. High-Severity Longitudinal and Transverse Cracking. 


\section{Patching and Utility Cut Patching}

\section{Description}

A patch is an area of pavement that has been replaced with new material to repair the existing pavement. A patch is considered a defect no matter how well it is performing (a patched area or adjacent area usually does not perform as well as an original pavement section). Generally, some roughness is associated with this distress.

\section{Severity Levels}

L Patch is in good condition and satisfactory. Ride quality is rated as low severity or better (Figure 11a).

M Patch is moderately deteriorated and/or ride quality is rated as medium severity (Figure 11b).

H Patch is badly deteriorated and/or ride quality is rated as high severity. Needs replacement soon (Figure 11c).

\section{How to Measure}

Patching is rated in square feet of surface area. However, if a single patch has areas of differing severity, these areas should be measured and recorded separately. For example, a $2.5 \mathrm{~m} 2(27.0 \mathrm{sq}$. ft) patch may have $1 \mathrm{~m} 2(11.0$ sq. $\mathrm{ft}$ ) of medium severity and $1.5 \mathrm{~m} 2$ (16.0 sq. ft) of low severity. These areas would be recorded separately. Any distress found in a patched area will not be recorded; however, its effect on the patch will be considered when determining the patch's severity level. No other distresses (e.g., shoving and cracking) are recorded within a patch; even if the patch material is shoving or cracking, the area is rated only as a patch. If a large amount of pavement has been replaced, it should not be recorded as a patch, but considered as new pavement (e.g., replacement of a complete intersection). 


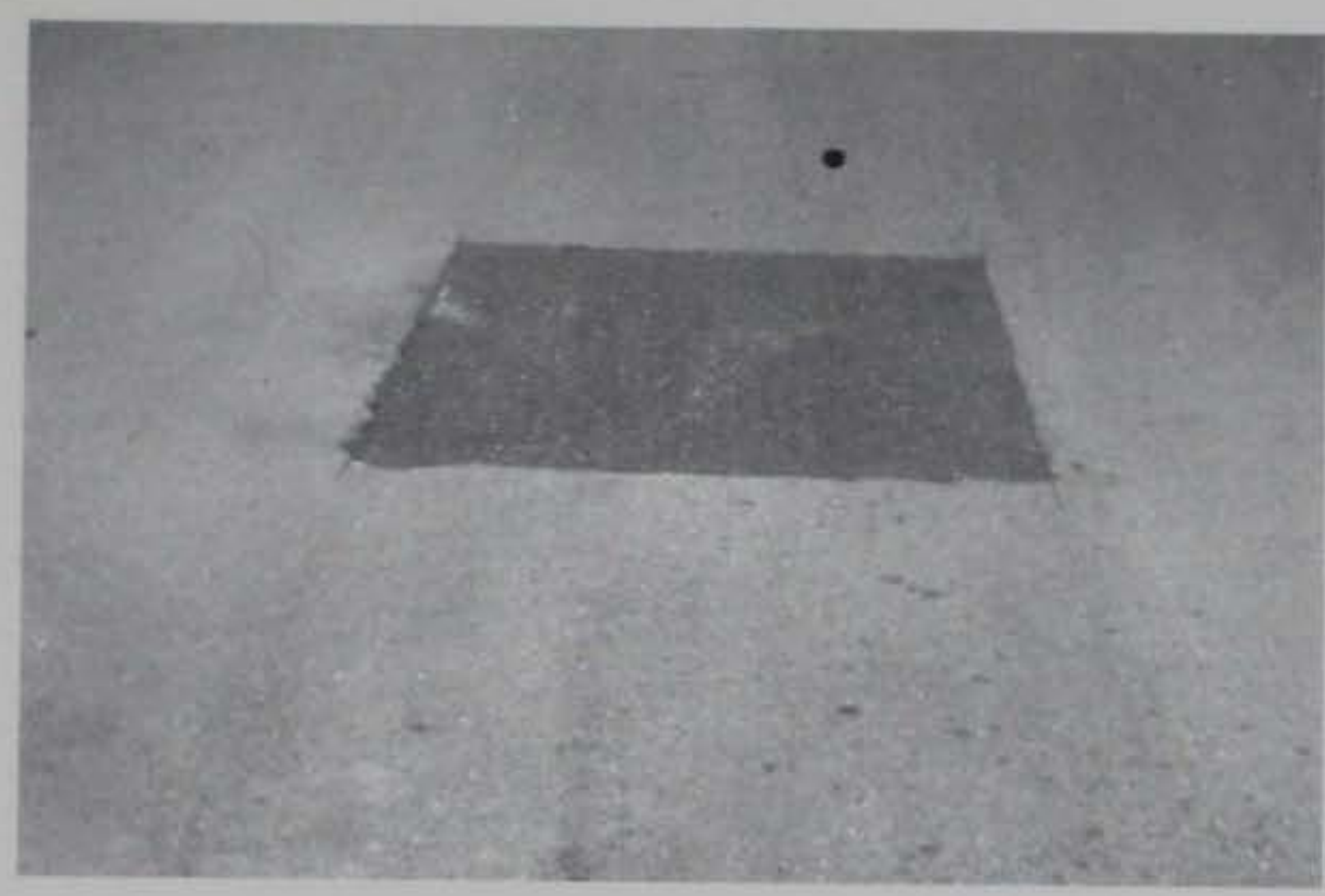

Figure 11a. Low-Severity Patching and Utility Cut Patching.

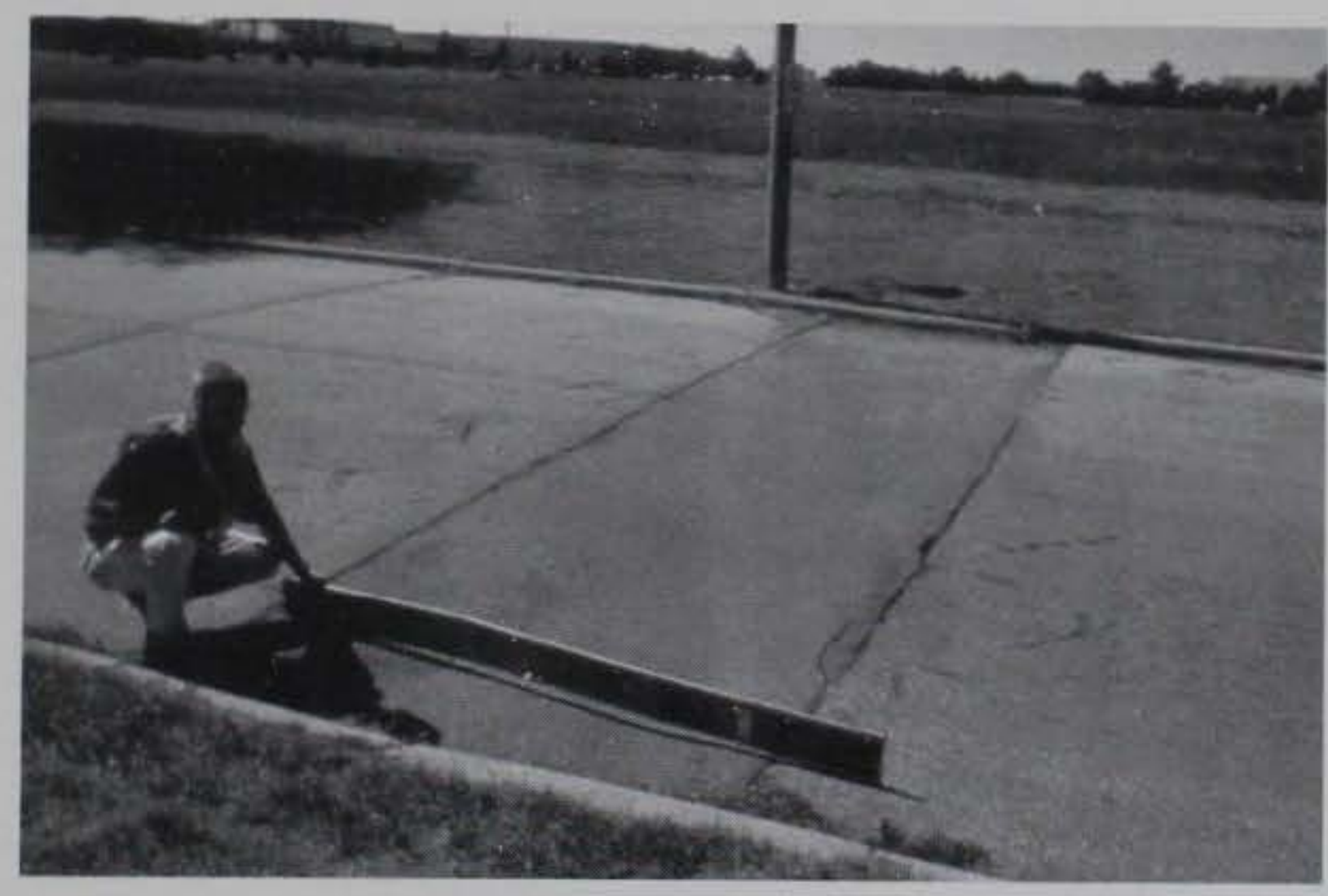

0
0
0
0

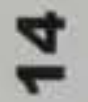

Figure 11b. Medium-Severity Patching and Utility Cut Patching.

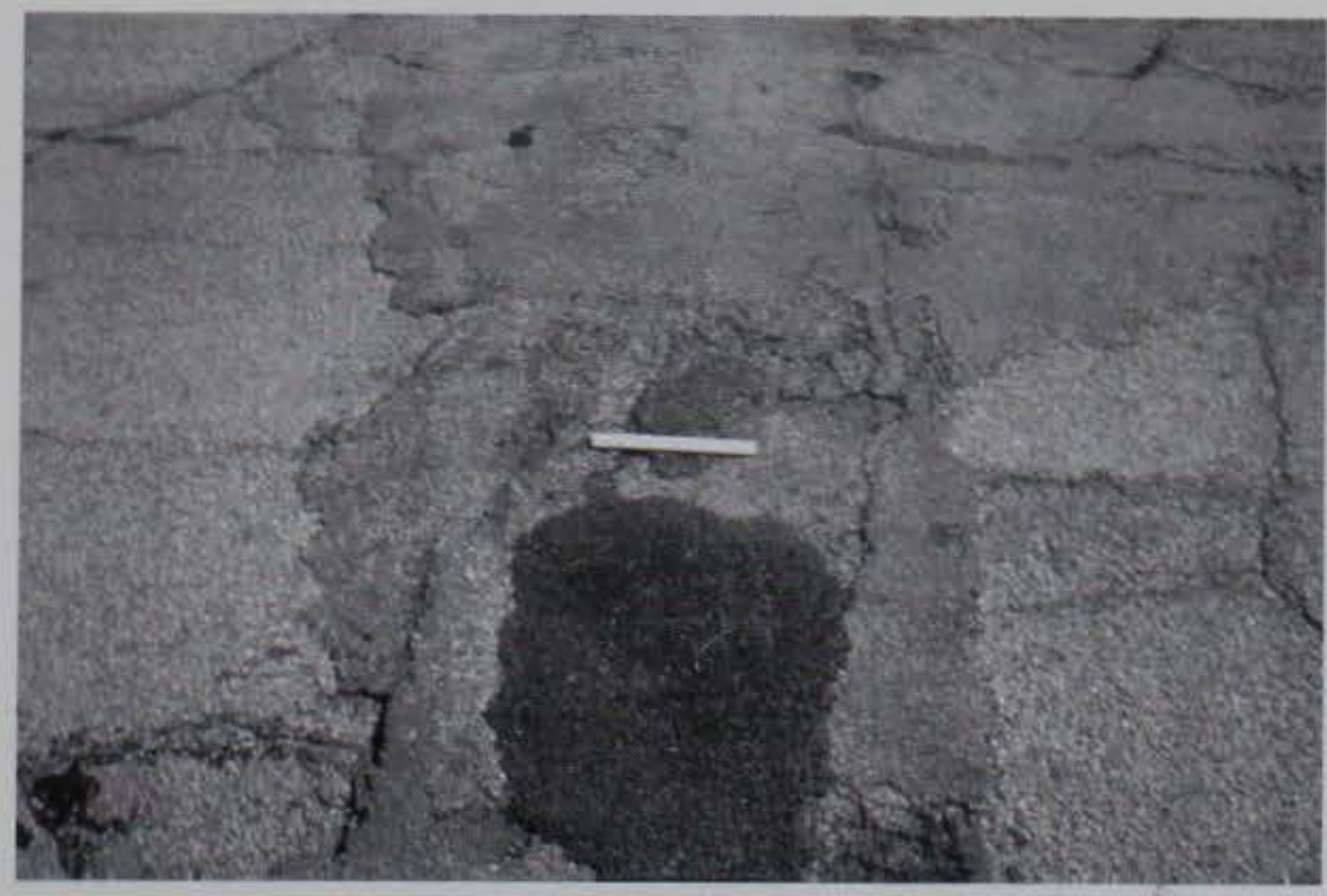

Figure 11c. High-Severity Patching and Utility Cut Patching. 


\section{Polished Aggregate}

\section{Description}

This distress is caused by repeated traffic applications. Polished aggregate is present when close examination of a pavement reveals that the portion of aggregate extending above the asphalt is either very small, or there are no rough or angular aggregate particles to provide good skid resistance. When the aggregate in the surface becomes smooth to the touch, adhesion with vehicle tires is considerably reduced. When the portion of aggregate extending above the surface is small, the pavement texture does not significantly contribute to reducing vehicle speed. Polished aggregate should be counted when close examination reveals that the aggregate extending above the asphalt is negligible, and the surface aggregate is smooth to the touch. This type of distress is indicated when the number on a skid resistance test is low or has dropped significantly from a previous rating.

\section{Severity Levels}

No degrees of severity are defined. However, the degree of polishing should be clearly evident in the sample unit in that the aggregate surface should be smooth to the touch. (Figure 12).

\section{How to Measure}

Polished aggregate is measured in square meters (feet) of surface area. If bleeding is counted, polished aggregate should not be counted. 


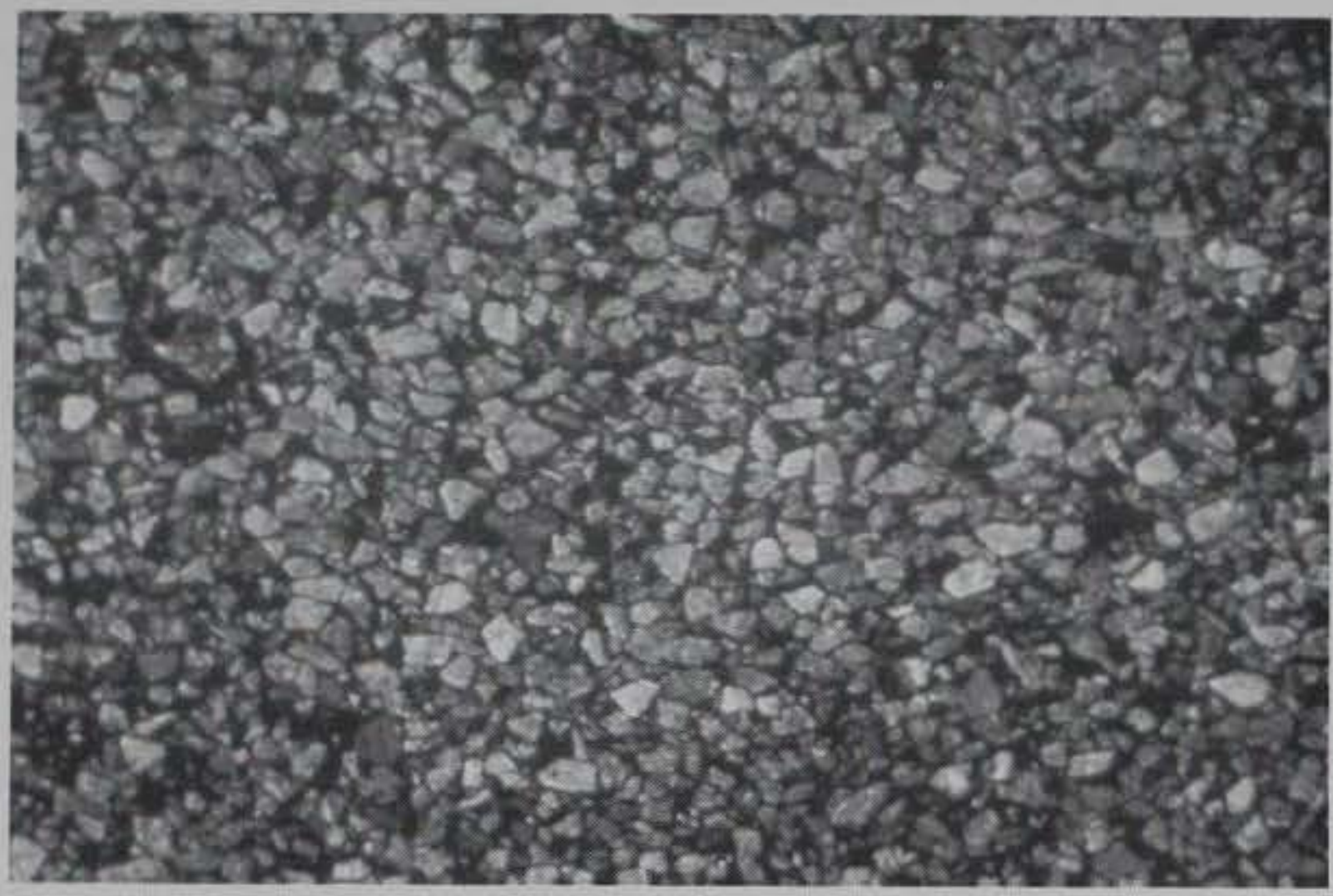

2

เก

Figure 12. Polished Aggregate.

$\frac{1}{2}$

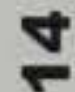

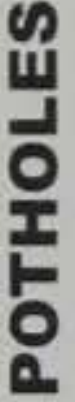

m

$\frac{2}{\frac{1}{7}}$

N

$\frac{2}{2}$

c 


\section{Potholes}

\section{Description}

Potholes are small-usually less than $750 \mathrm{~mm}$ (30 in.) in diameterbowl-shaped depressions in the pavement surface. They generally have sharp edges and vertical sides near the top of the hole. When holes are created by high-severity alligator cracking, they should be identified as potholes, not as weathering.

\section{Severity Levels}

The levels of severity for potholes less than $750 \mathrm{~mm}$ (30 in.) in diameter are based on both the diameter and the depth of the pothole, according to Table 1.

Table 1. Levels of Severity for Potholes.

\begin{tabular}{|c|c|c|c|}
\hline \multirow[b]{2}{*}{$\begin{array}{l}\text { Maximum Depth of } \\
\text { Pothole }\end{array}$} & \multicolumn{3}{|c|}{$\begin{array}{l}\text { Average Diameter (mm) } \\
\text { (in.) }\end{array}$} \\
\hline & $\begin{array}{l}100 \text { to } 200 \mathrm{~mm} \\
\text { ( } 4 \text { to } 8 \mathrm{in.} \text { ) }\end{array}$ & $\begin{array}{l}200 \text { to } 450 \mathrm{~mm} \\
\text { (8 to } 18 \mathrm{in.})\end{array}$ & $\begin{array}{l}450 \text { to } 750 \mathrm{~mm} \\
\text { (18 to } 30 \text { in.) }\end{array}$ \\
\hline $\begin{array}{c}13 \text { to } \leq 25 \mathrm{~mm} \\
(1 / 2 \text { to } 1 \mathrm{in} .)\end{array}$ & $\mathrm{L}$ & $\mathrm{L}$ & $M$ \\
\hline $\begin{array}{c}>25 \text { and } \leq 50 \mathrm{~mm} \\
(1 \text { to } 2 \text { in.) }\end{array}$ & $\mathrm{L}$ & $M$ & $\mathrm{H}$ \\
\hline $\begin{aligned}> & 50 \mathrm{~mm} \\
& (2 \mathrm{in} .)\end{aligned}$ & $M$ & $M$ & $\mathrm{H}$ \\
\hline
\end{tabular}

If the pothole is more than $750 \mathrm{~mm}$ (30 in.) in diameter, the area should be determined in square feet and divided by $0.5 \mathrm{~m}^{2}(5.5 \mathrm{sq} \mathrm{ft})$ to find the equivalent number of holes. If the depth is $25 \mathrm{~mm}$ ( 1 in.) or less, the holes are considered medium severity. If the depth is more than $25 \mathrm{~mm}$ (1 in.), they are considered high severity (Figures 13a through 13c).

\section{How to Measure}

Potholes are measured by counting the number that are low, medium, and high severity and recording them separately. 


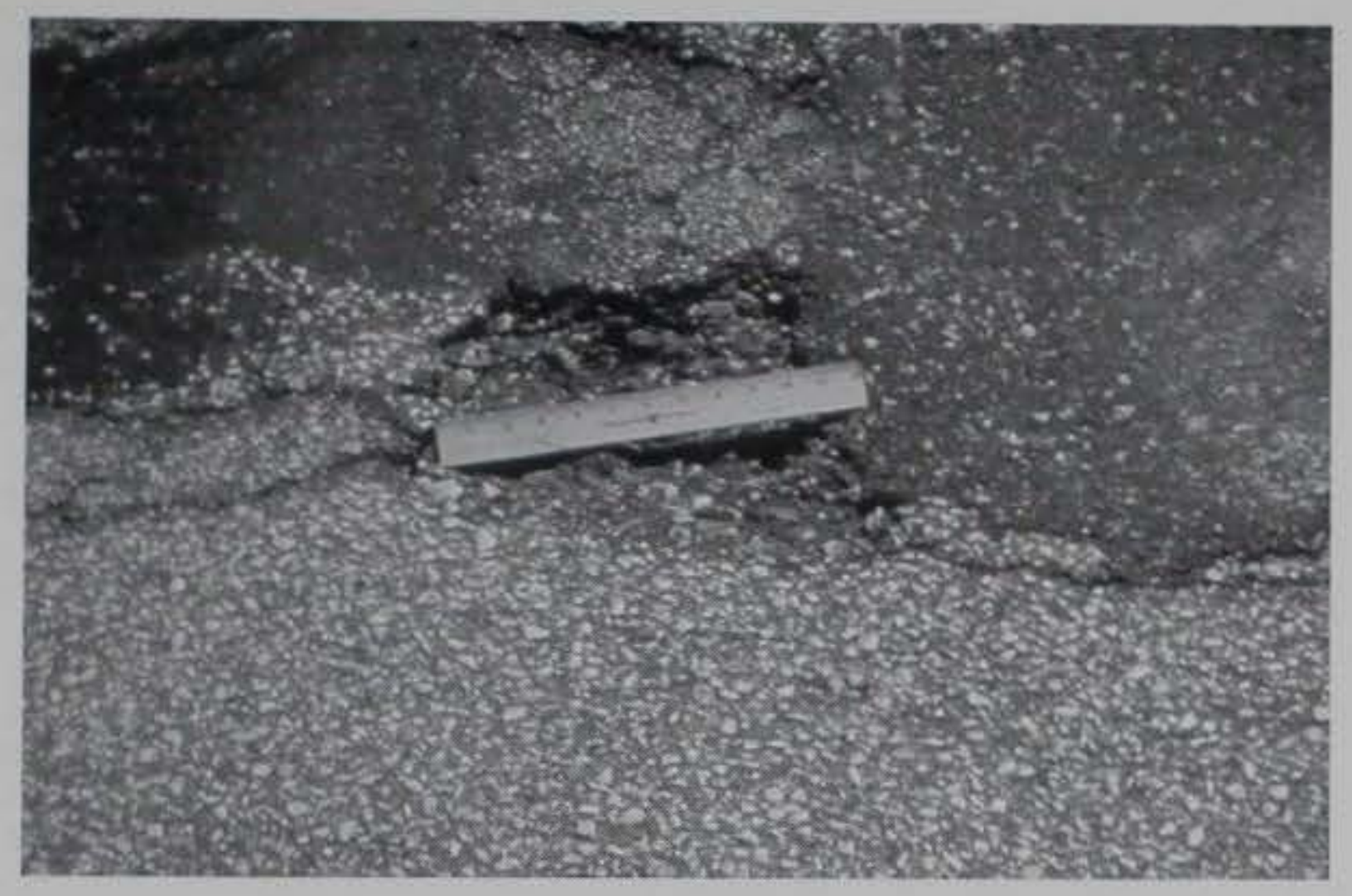

Figure 13a. Low-Severity Pothole.

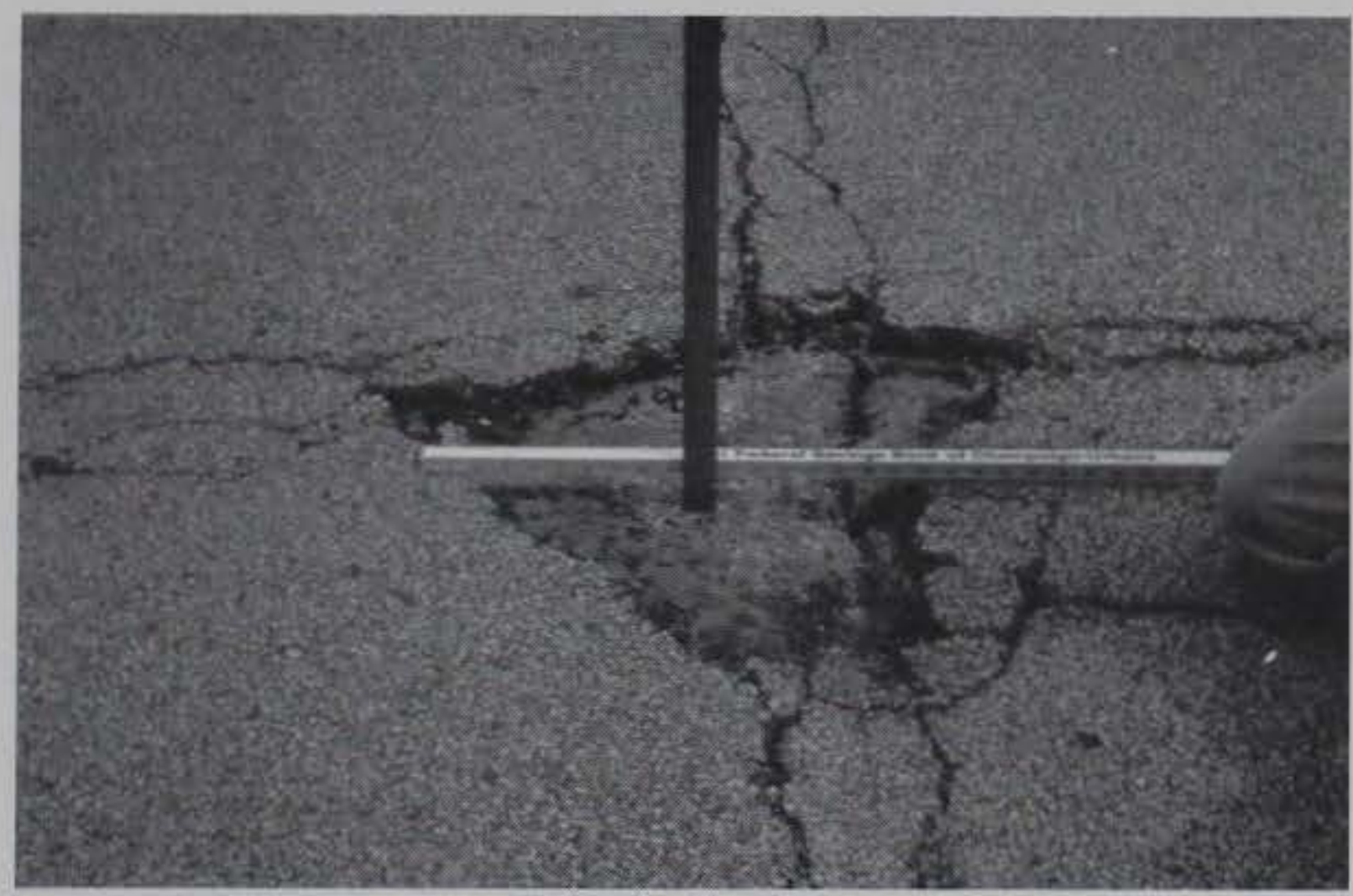

Figure 13b. Medium-Severity Pothole.

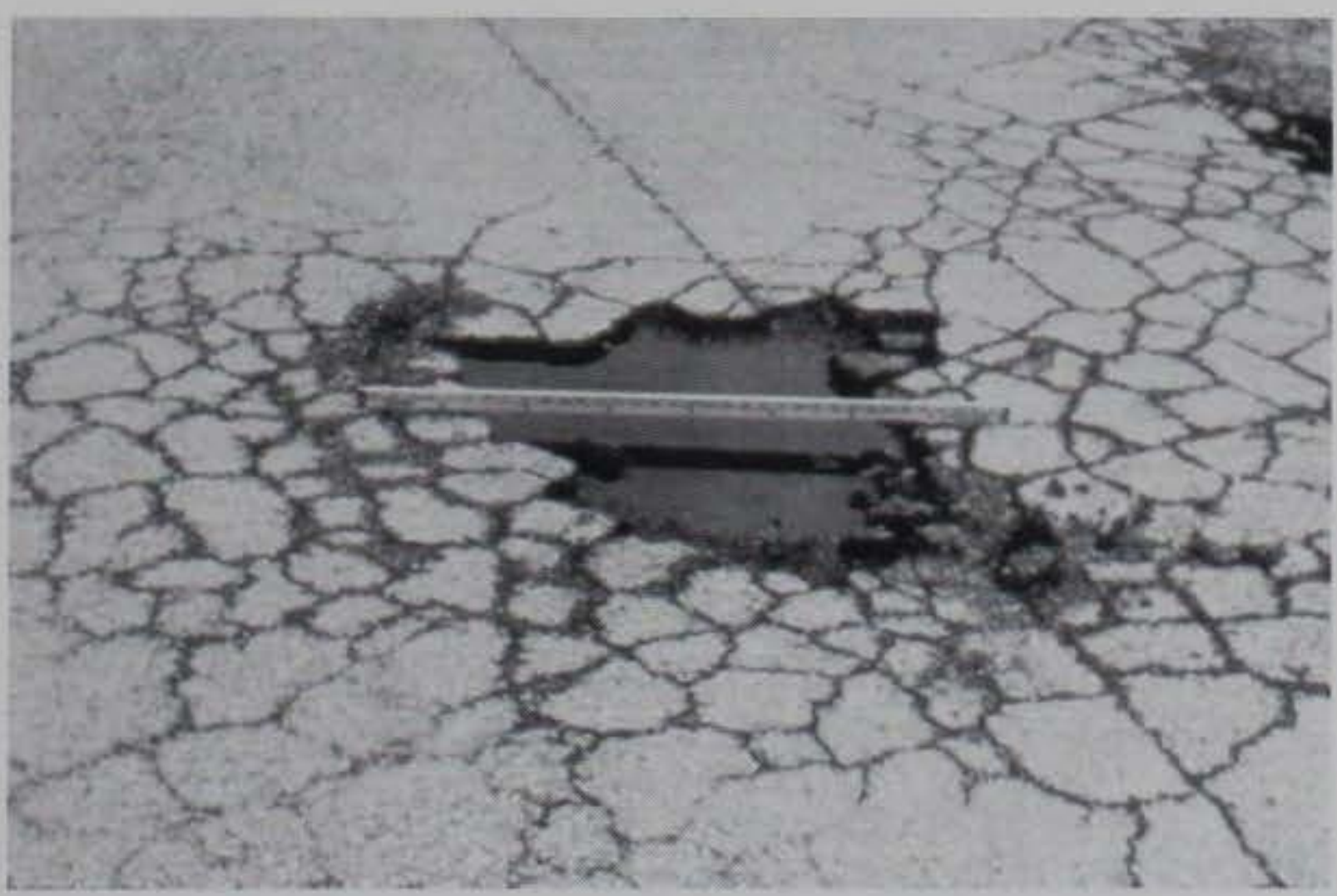

0
111
0
0
0
0

Figure 13c. High-Severity Pothole.

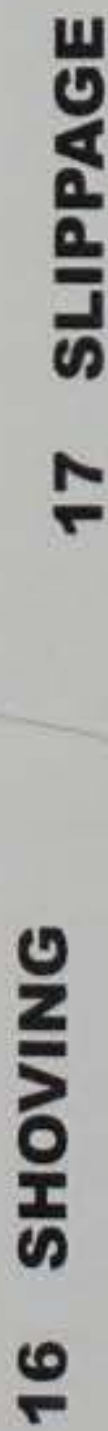




\section{Railroad Crossing}

\section{Description}

Railroad crossing defects are depressions or bumps around and/or between tracks.

\section{Severity Levels}

L Railroad crossing causes low-severity ride quality (Figure 14a).

M Railroad crossing causes medium-severity ride quality (Figure 14b).

H Railroad crossing causes high-severity ride quality (Figure 14c).

\section{How to Measure}

The area of the crossing is measured in square meters (feet) of surface area. If the crossing does not affect ride quality, it should not be counted. Any large bump created by the tracks should be counted as part of the crossing. 


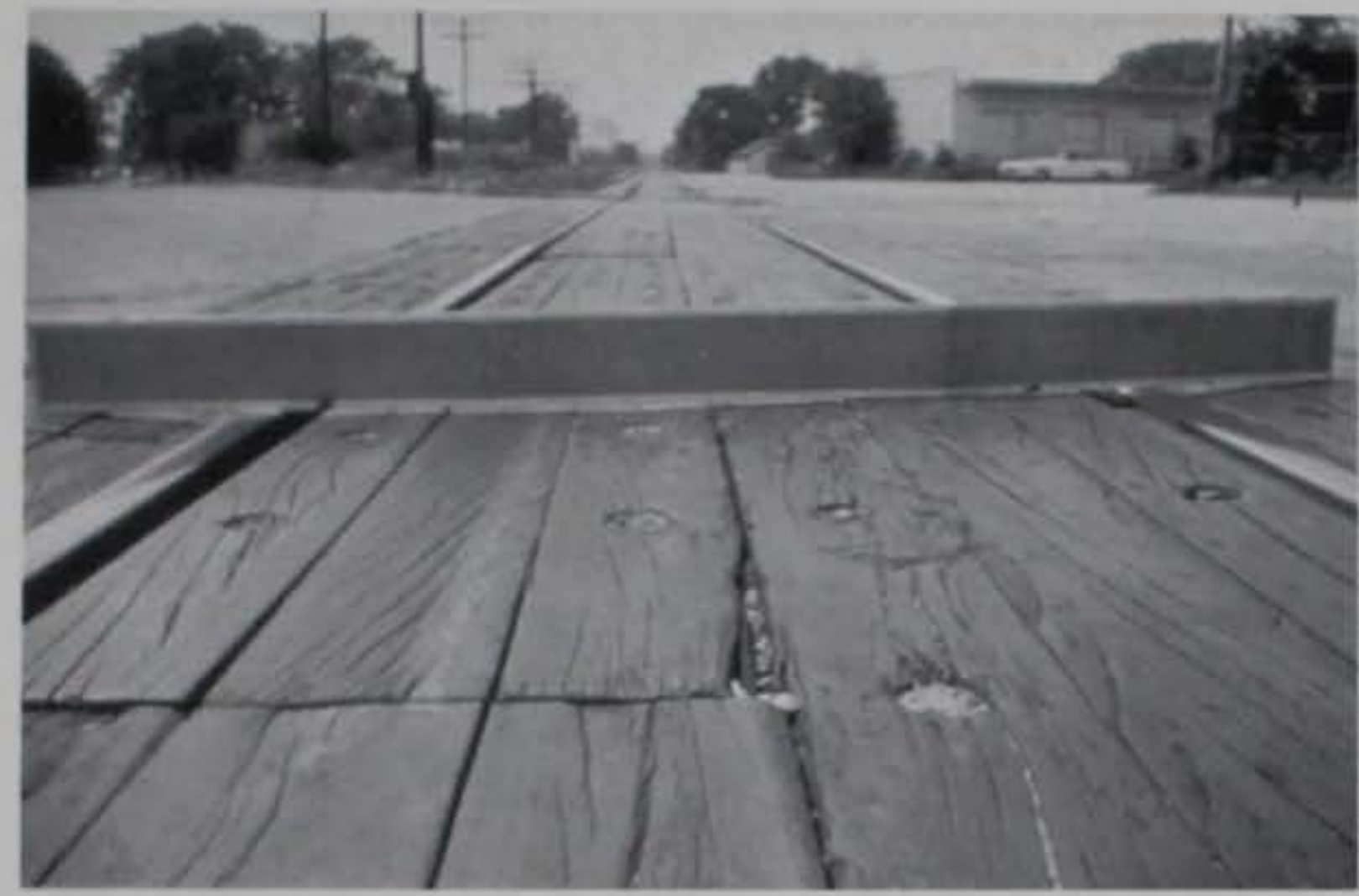

Figure 14a. Low-Severity Railroad Crossing.

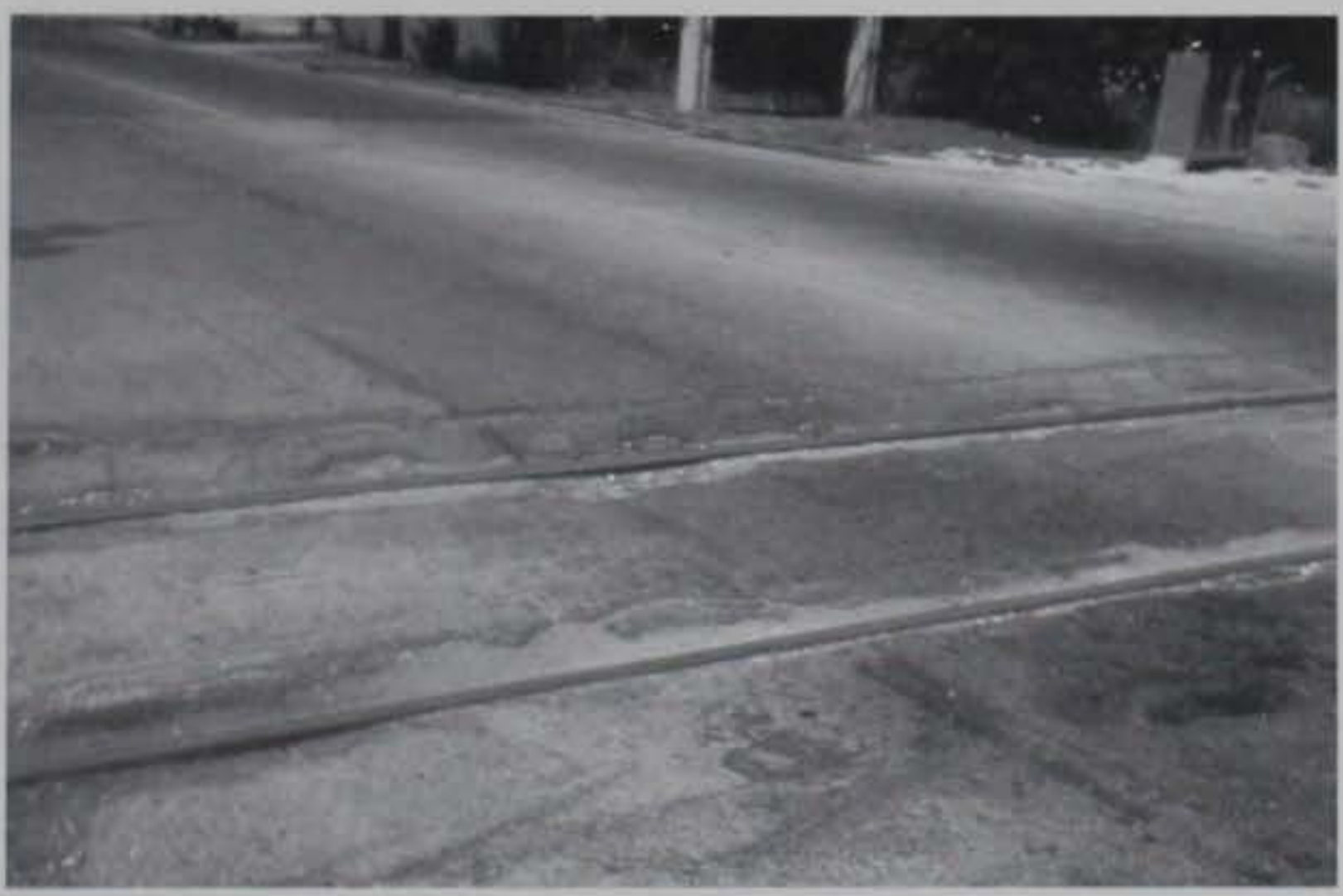

$\frac{1}{8}$

$\stackrel{2}{+2}$

Figure 14b. Medium-Severity Railroad Crossing.

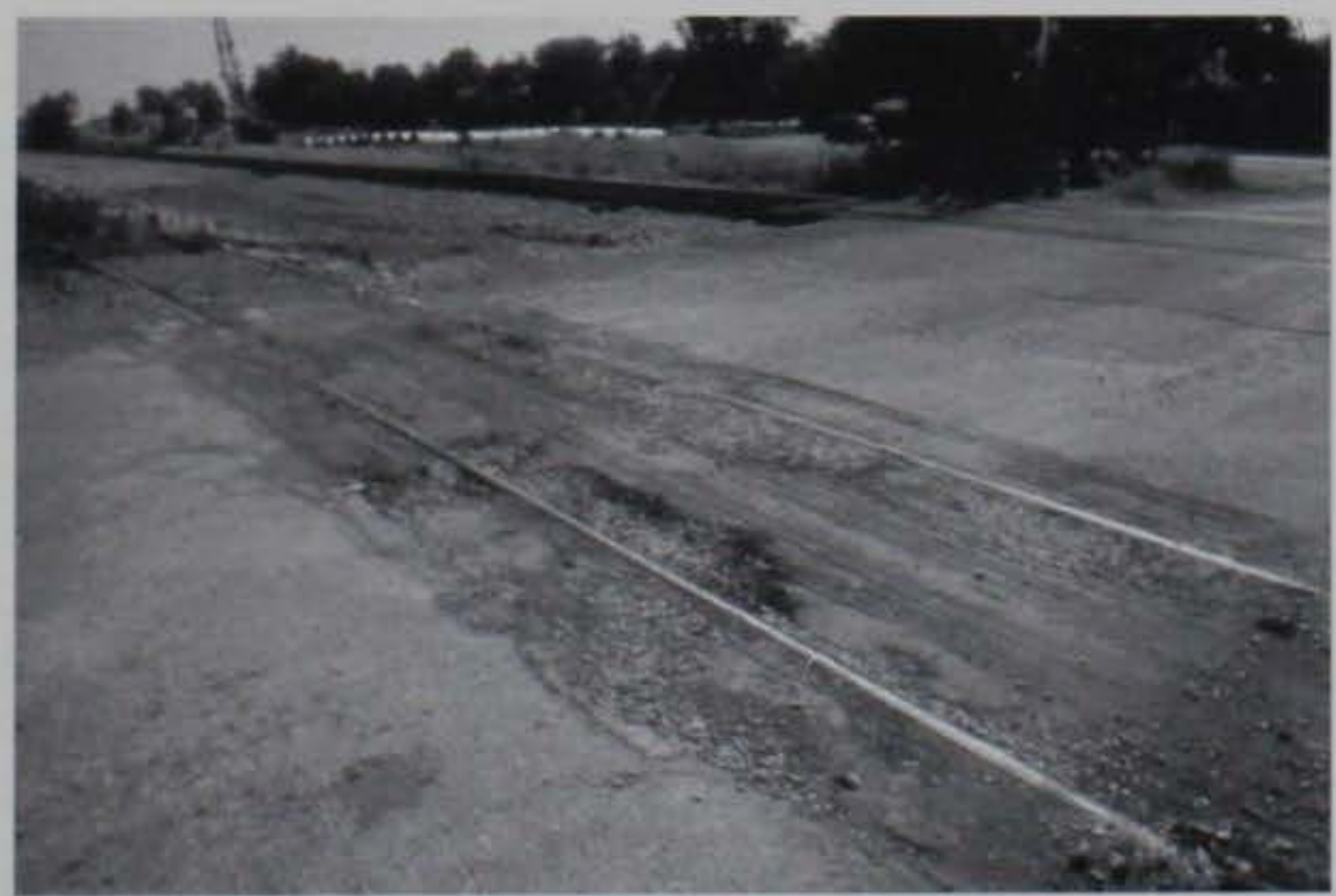

Figure 14c. High-Severity Railroad Crossing. 


\section{Rutting}

\section{Description}

A rut is a surface depression in the wheel paths. Pavement uplift may occur along the sides of the rut, but, in many instances, ruts are noticeable only after a rainfall when the paths are filled with water. Rutting stems from a permanent deformation in any of the pavement layers or subgrades, usually caused by consolidated or lateral movement of the materials due to traffic load.

\section{Severity Levels}

Mean Rut Depth

L 6 to $13 \mathrm{~mm}(1 / 4$ to $1 / 2 \mathrm{in}$.) (Figure $15 \mathrm{a})$.

M $>13$ to $25 \mathrm{~mm}$ ( $>1 / 2$ to $1 \mathrm{in}$.) (Figure $15 \mathrm{~b}$ ).

H $>25 \mathrm{~mm}$ (>1 in.) (Figure 15c).

\section{How to Measure}

Rutting is measured in square meters (feet) of surface area and its severity is determined by the mean depth of the rut (see above). The mean rut depth is calculated by laying a straight edge across the rut, measuring its depth, then using measurements taken along the length of the rut to compute its mean depth in millimeters. 


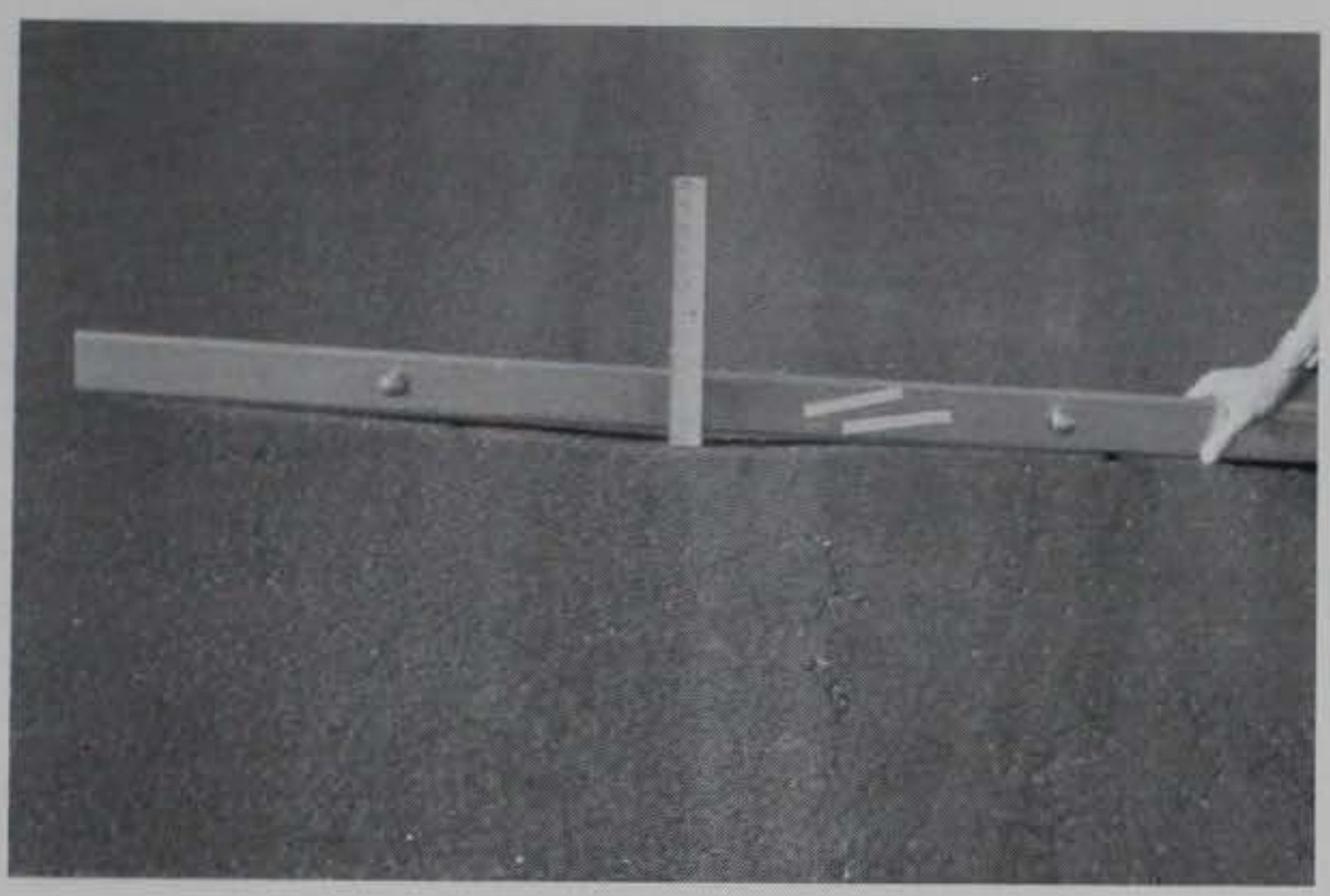

2

คิ

Figure 15a. Low-Severity Rutting.

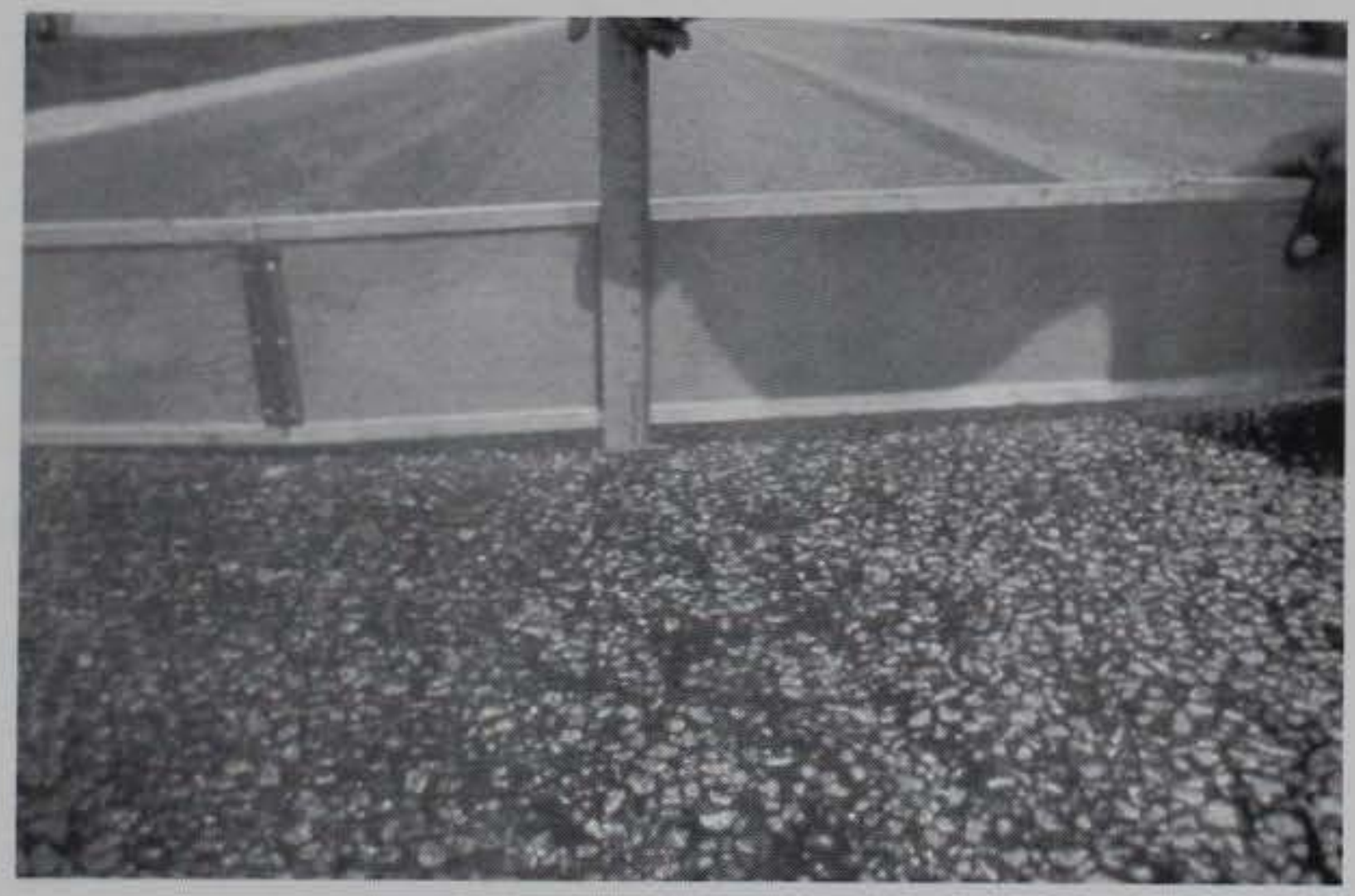

늘

구

$\frac{1}{11}$



Figure 15b. Medium-Severity Rutting.

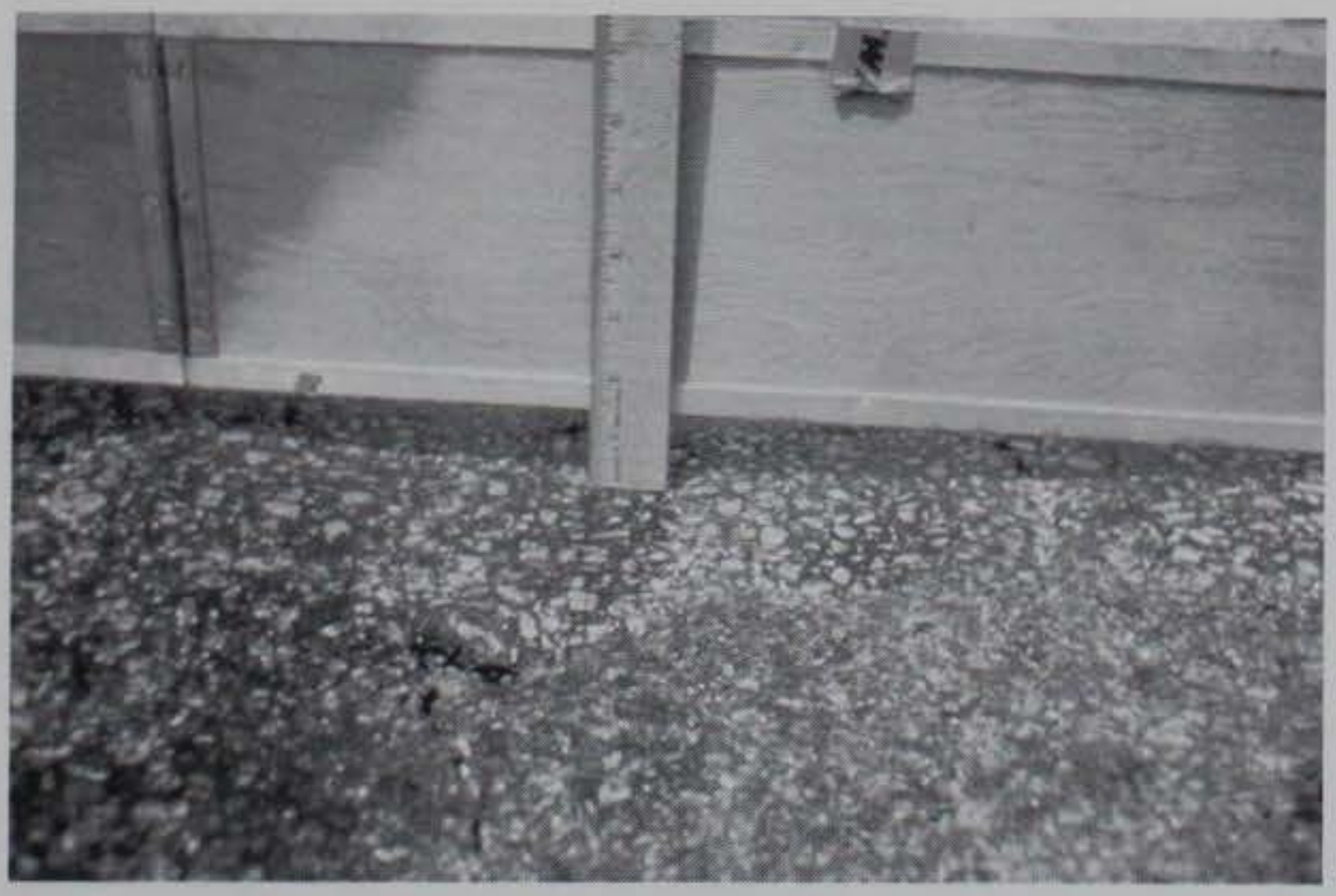

บ1

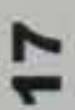

Figure 15c. High-Severity Rutting.

일 


\section{Shoving}

\section{Description}

Shoving is a permanent, longitudinal displacement of a localized area of the pavement surface caused by traffic loading. When traffic pushes against the pavement, it produces a short, abrupt wave in the pavement surface. This distress normally occurs only in unstable liquid asphalt mix (cutback or emulsion) pavements.

Shoves also occur where asphalt pavements abut PCC pavements; the PCC pavements increase in length and push the asphalt pavement, causing the shoving.

\section{Severity Levels}

L Shove causes low-severity ride quality (Figure 16a).

M Shove causes medium-severity ride quality (Figure 16b).

H Shove causes high-severity ride quality (Figure 16c).

\section{How to Measure}

Shoves are measured in square meters (feet) of surface area. Shoves occurring in patches are considered in rating the patch, not as a separate distress. 


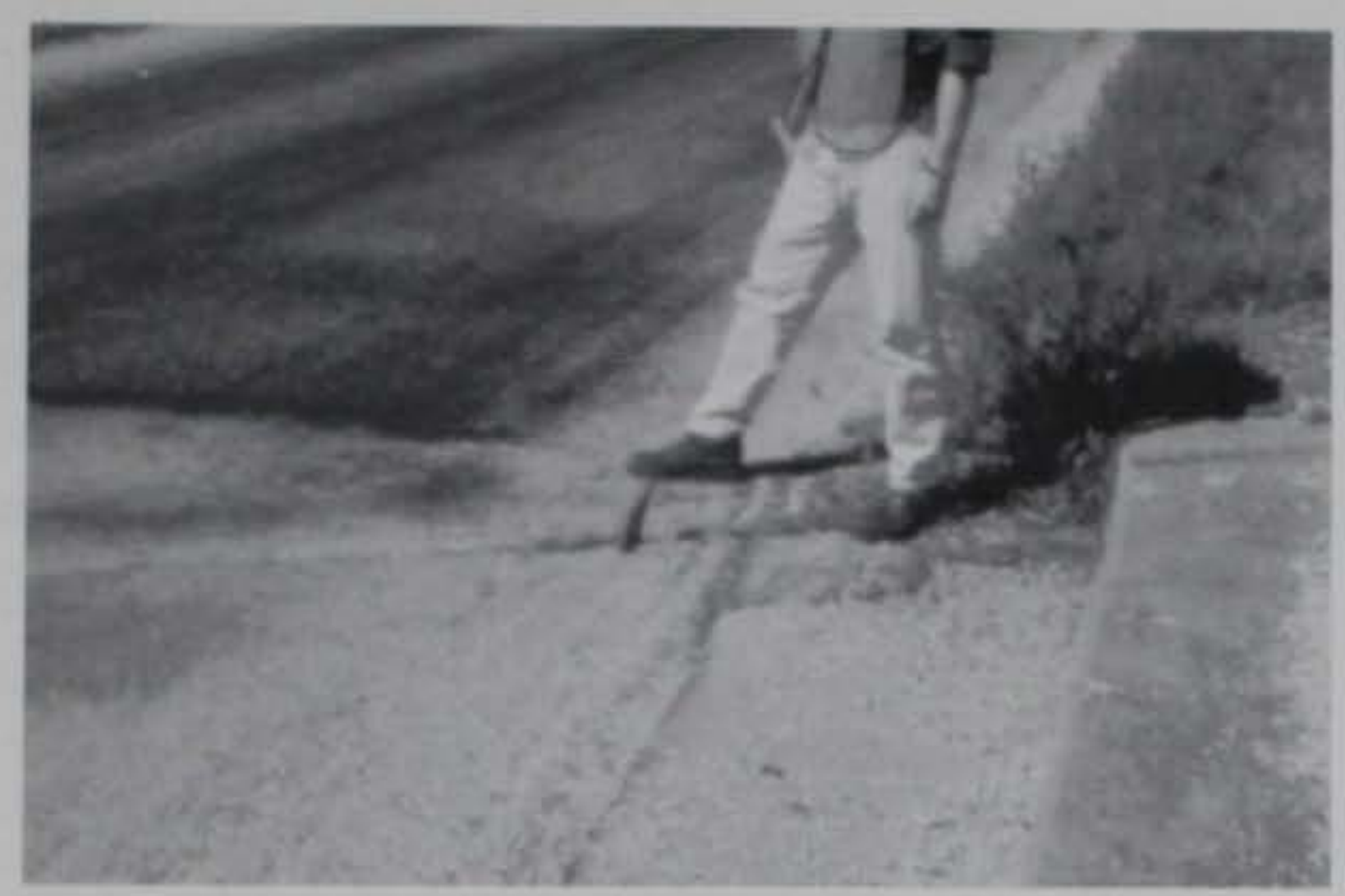

Figure 16a. Low-Severity Shoving.

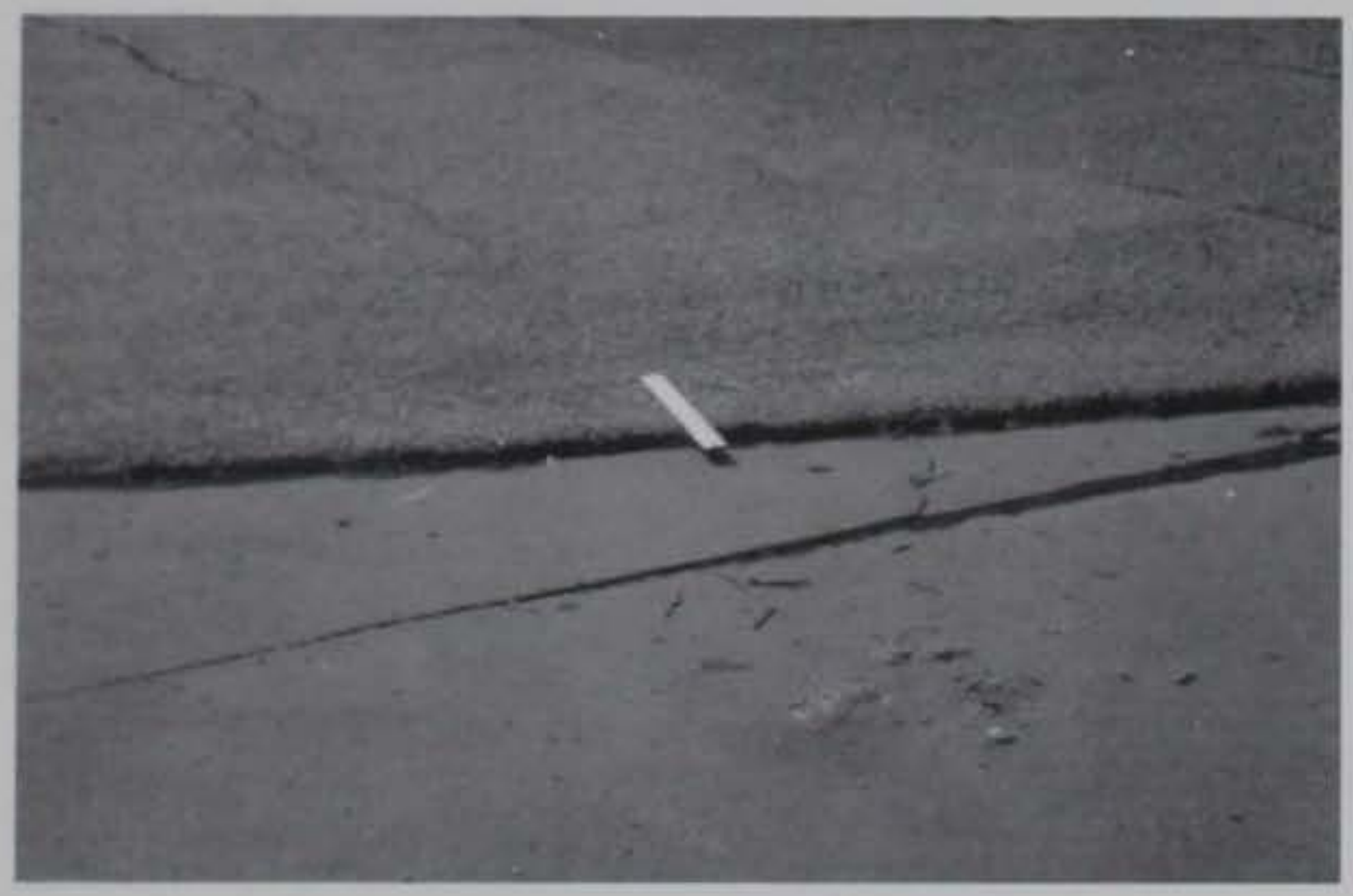

$\frac{0}{\frac{3}{2}}$

$\stackrel{\circ}{\circ}$

$\frac{\text { 嵅 }}{3}$

$\stackrel{\infty}{\leftarrow}$

Figure 16b. Medium-Severity Shoving.

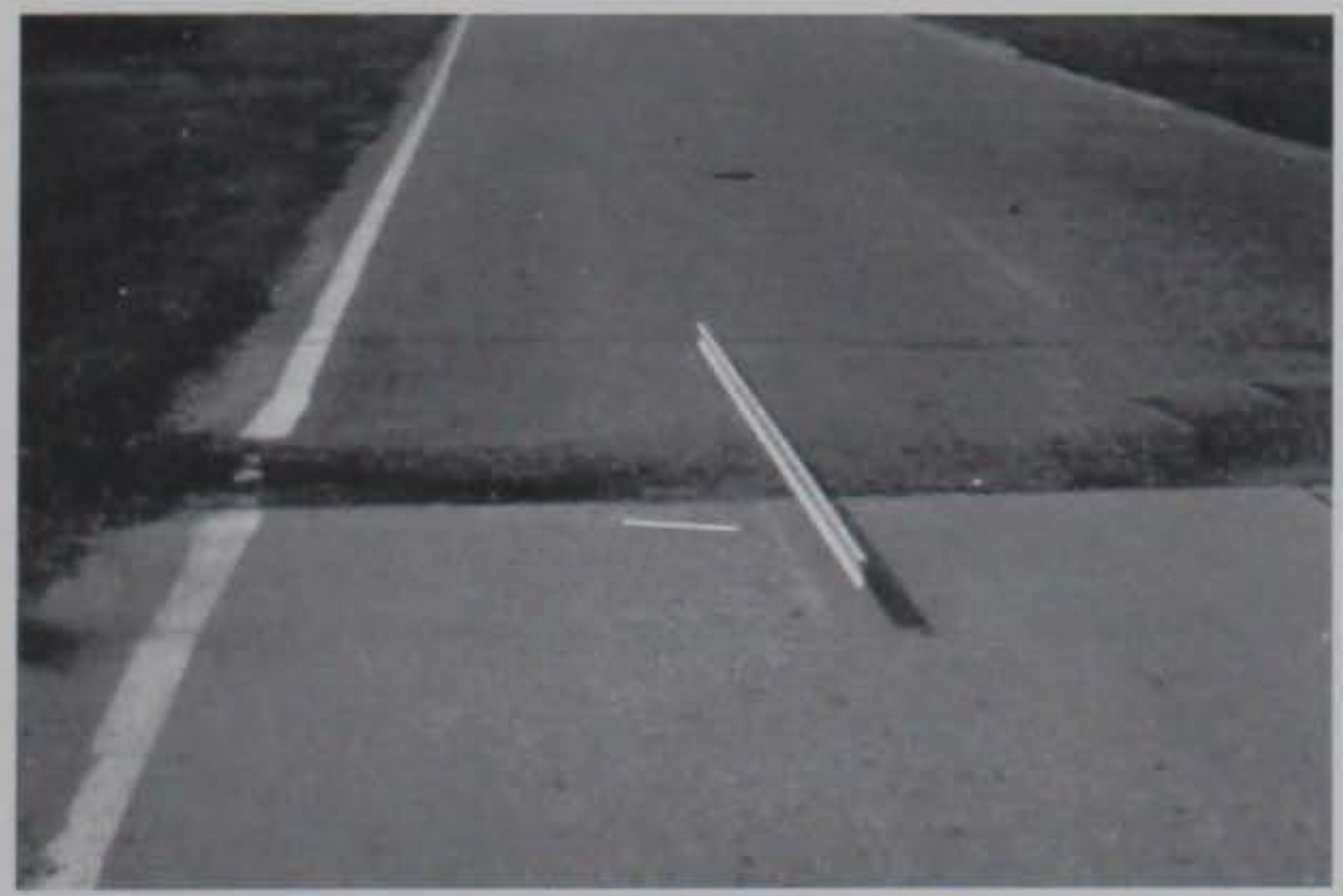

峁

Figure 16c. High-Severity Shoving. 


\section{Slippage Cracking}

\section{Description}

Slippage cracks are crescent or half-moon shaped cracks, usually transverse to the direction of travel. They are produced when braking or turning wheels cause the pavement surface to slide or deform. This distress usually occurs in overlaps when there is a poor bond between the surface and the next layer of the pavement structure.

\section{Severity Level}

L Average crack width is $<10 \mathrm{~mm}$ (3/8 in.) (Figure 17a).

M One of the following conditions exists (Figure 17b): (1) average crack width is $\geq 10$ and $<40 \mathrm{~mm}$ ( $\geq 3 / 8$ and $<1-1 / 2$ in.); (2) the area around the crack is moderately spalled and/or surrounded with secondary cracks..

H One of the following conditions exists (Figure 17c): (1) the average crack width is $>40 \mathrm{~mm}(1-1 / 2 \mathrm{in}$.), or (2) the area around the crack is broken into easily removed pieces.

\section{How to Measure}

The area associated with a given slippage crack is measured in square meters (feet) and rated according to the highest level of severity in the area. 


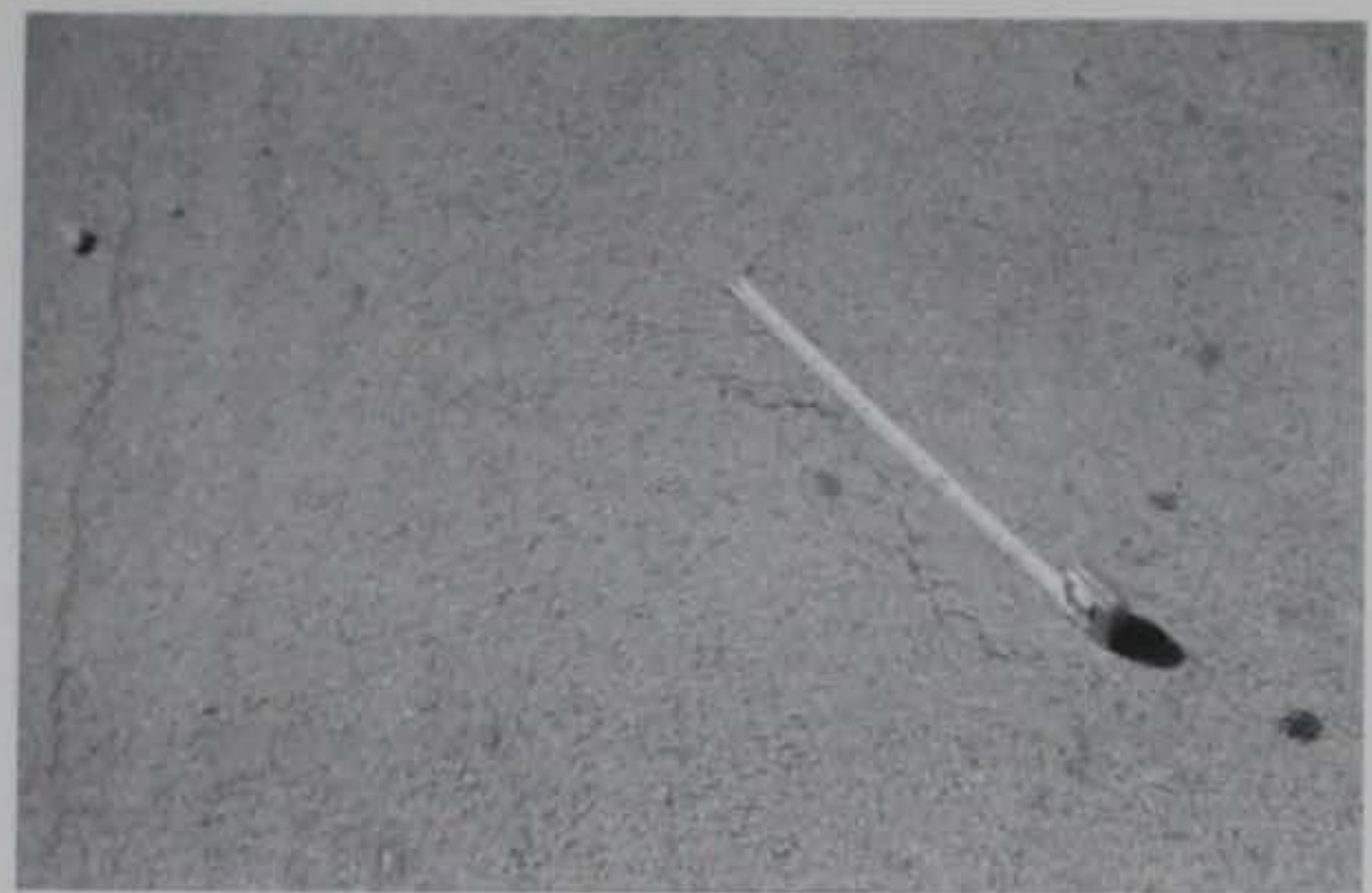

Figure 17a. Low-Severity Slippage Cracking.

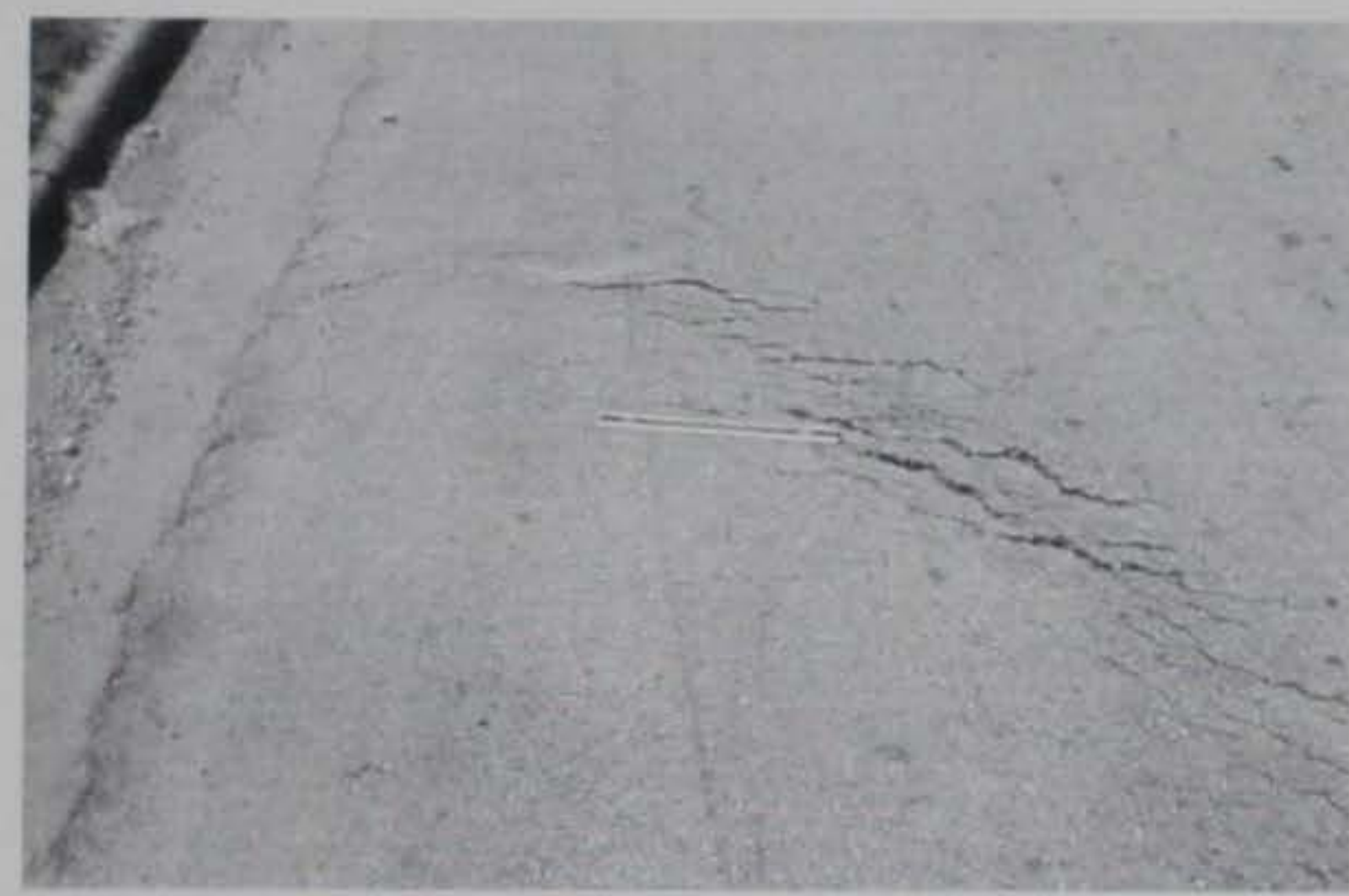

Figure 17b. Medium-Severity Slippage Cracking.

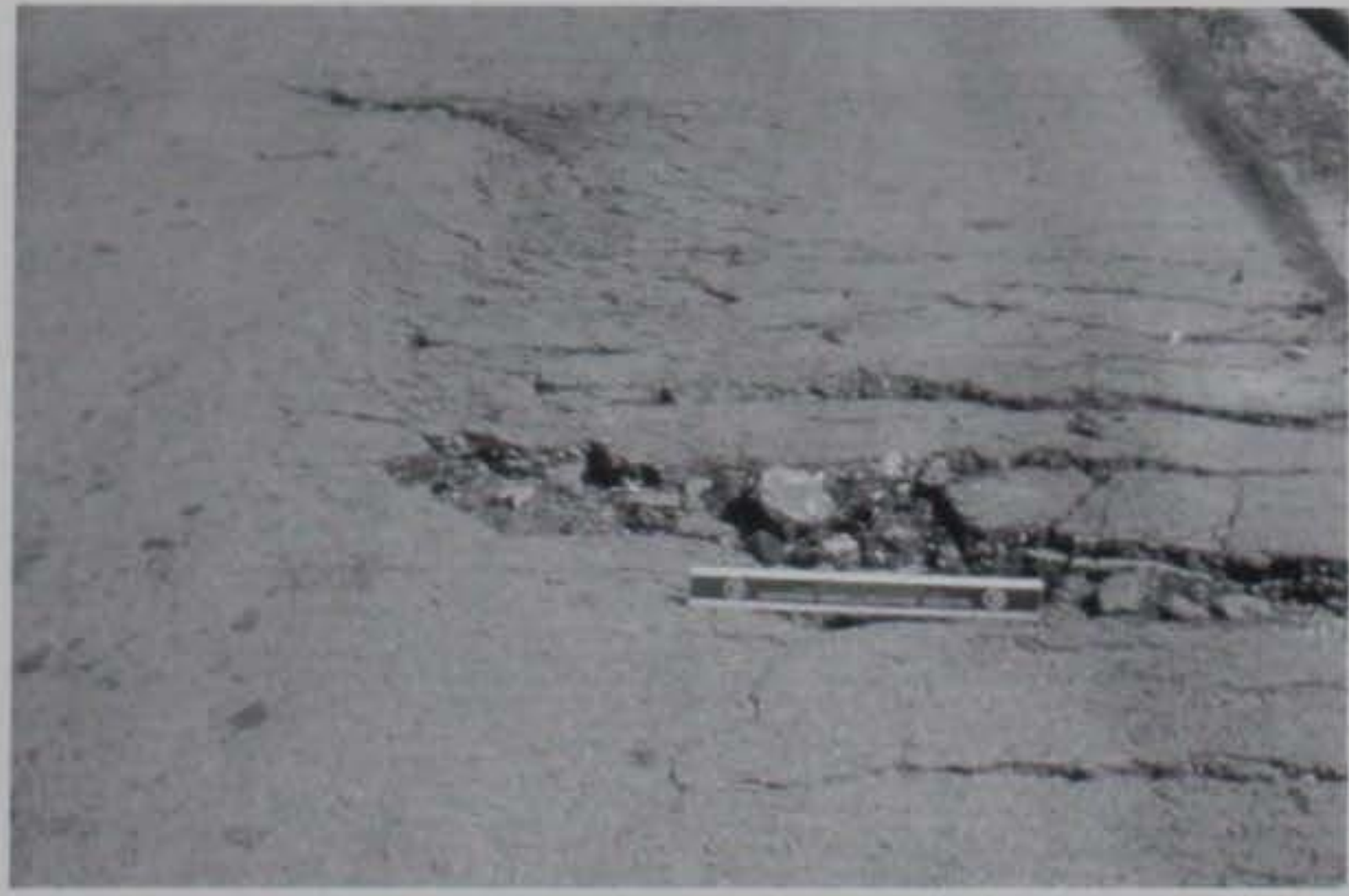

$\frac{1}{2}$

Figure 17c. High-Severity Slippage Cracking. 


\section{Swell}

\section{Description}

Swell is characterized by an upward bulge in the pavement's surface-a long, gradual wave more than $3 \mathrm{~m}$ (10 ft) long (Figure 18). Swelling can be accompanied by surface cracking. This distress is usually caused by frost action in the subgrade or by swelling soil.

\section{Severity Level}

L Swell causes low-severity ride quality. Low-severity swells are not always easy to see, but can be detected by driving at the speed limit over the pavement section. An upward motion will occur at the swell if it is present.

M Swell causes medium-severity ride quality.

H Swell causes high-severity ride quality.

\section{How to Measure}

The surface area of the swell is measured in square meters (feet). 


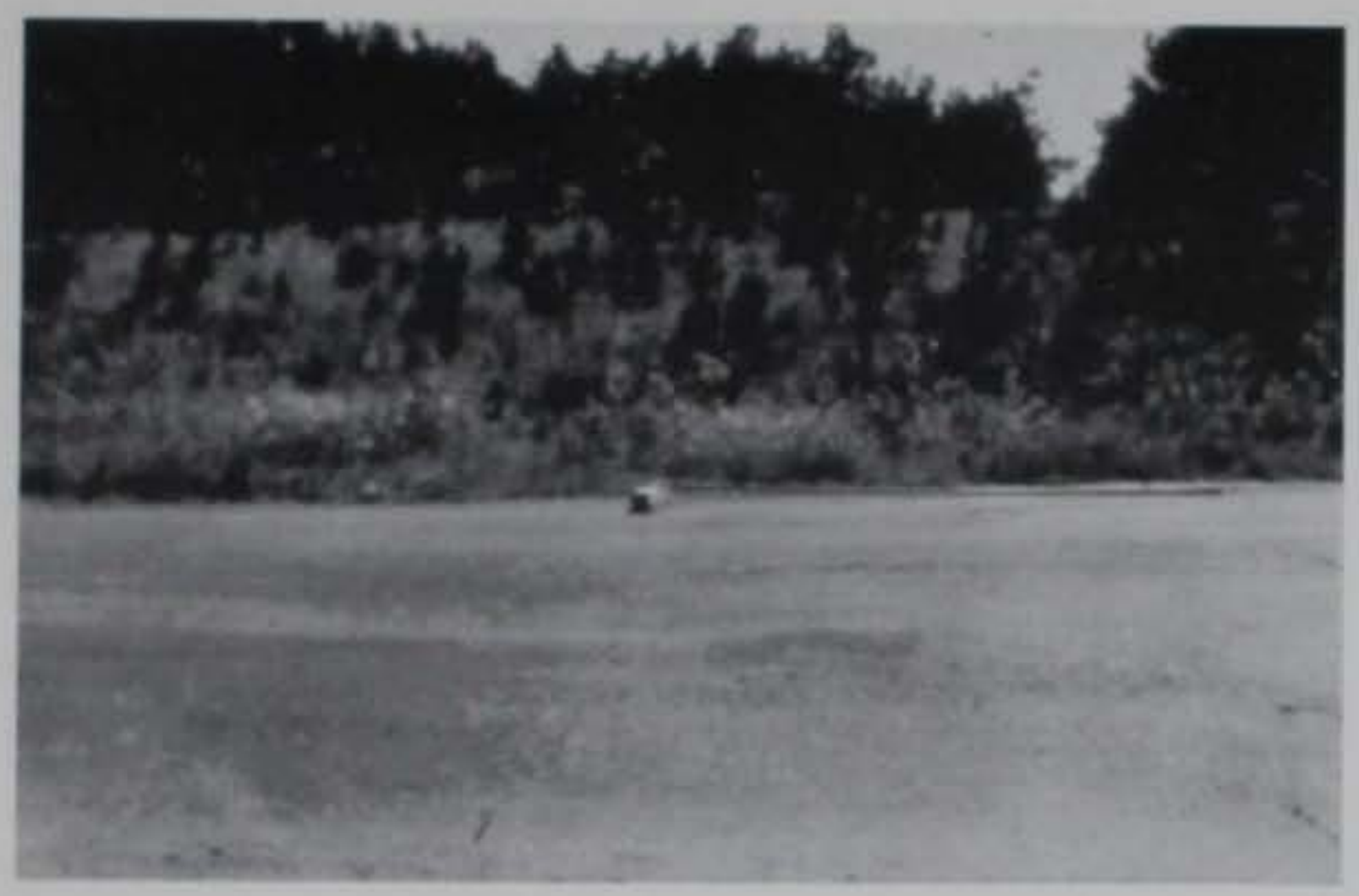

Figure 18. Example Swell. Severity level is based on ride quality criteria. 


\section{Weathering and Raveling}

\section{Description}

Weathering and raveling are the wearing away of the pavement surface due to a loss of asphalt or tar binder and dislodged aggregate particles. These distresses indicate that either the asphalt binder has hardened appreciably or that a poor-quality mixture is present. In addition, raveling may be caused by certain types of traffic, e.g., tracked vehicles. Softening of the surface and dislodging of the aggregates due to oil spillage are also included under raveling.

\section{Severity Levels}

L Aggregate or binder has started to wear away. In some areas, the surface is starting to pit (Figure 19a). In the case of oil spillage, the oil stain can be seen, but the surface is hard and cannot be penetrated with a coin.

M Aggregate or binder has worn away. The surface texture is moderately rough and pitted (Figure 19b). In the case of oil spillage, the surface is soft and can be penetrated with a coin.

H Aggregate or binder has been worn away considerably. The surface texture is very rough and severely pitted. The pitted areas are less than $10 \mathrm{~mm}(4 \mathrm{in}$.) in diameter and less than $13 \mathrm{~mm}(1 / 2$ in.) deep (Figure 19c); pitted areas larger than this are counted as potholes. In the case of oil spillage, the asphalt binder has lost its binding effect and the aggregate has become loose.

\section{How to Measure}

Weathering and raveling are measured in square meters (feet) of surface area. 


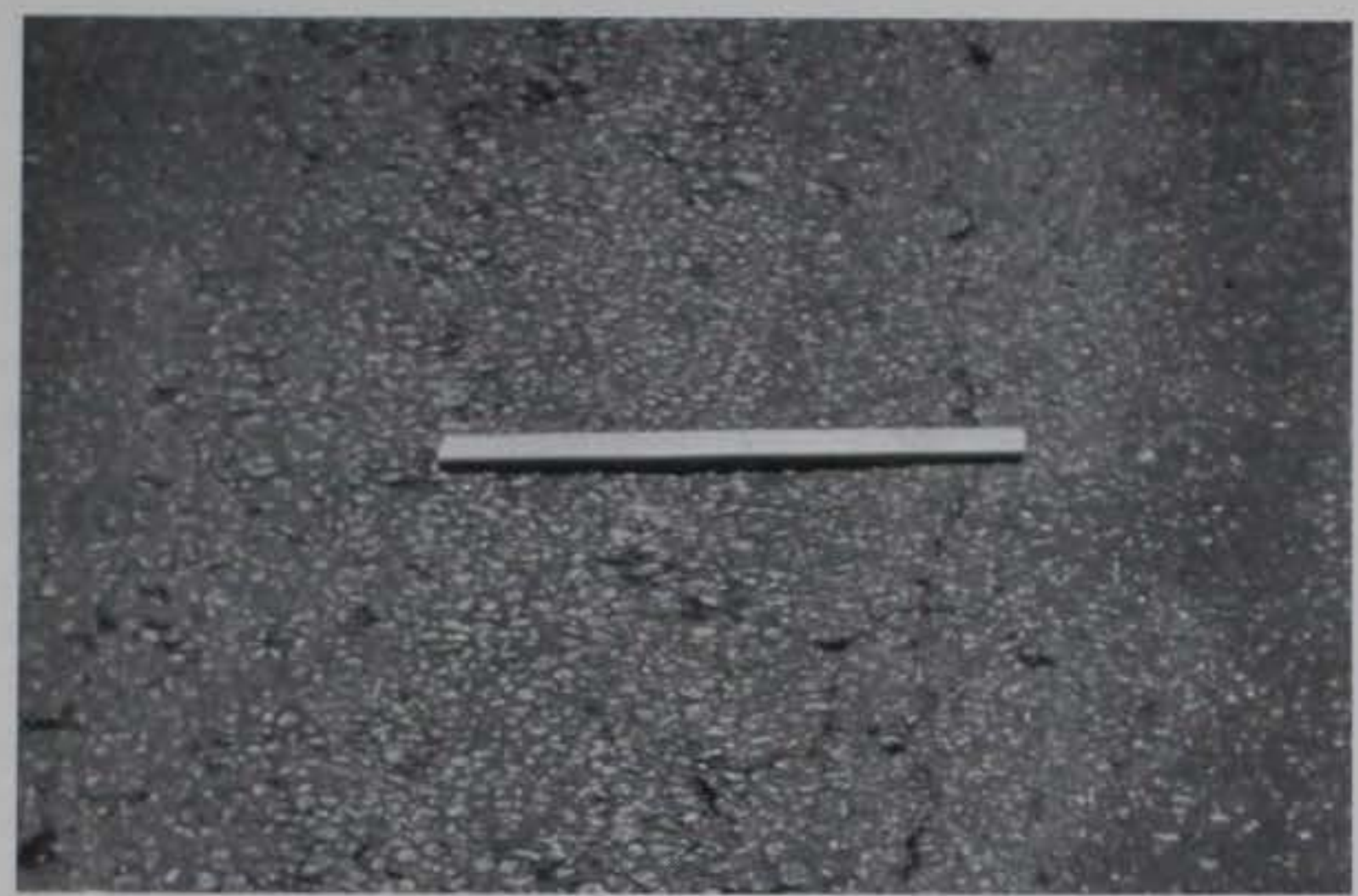

Figure 19a. Low-Severity Weathering and Raveling.

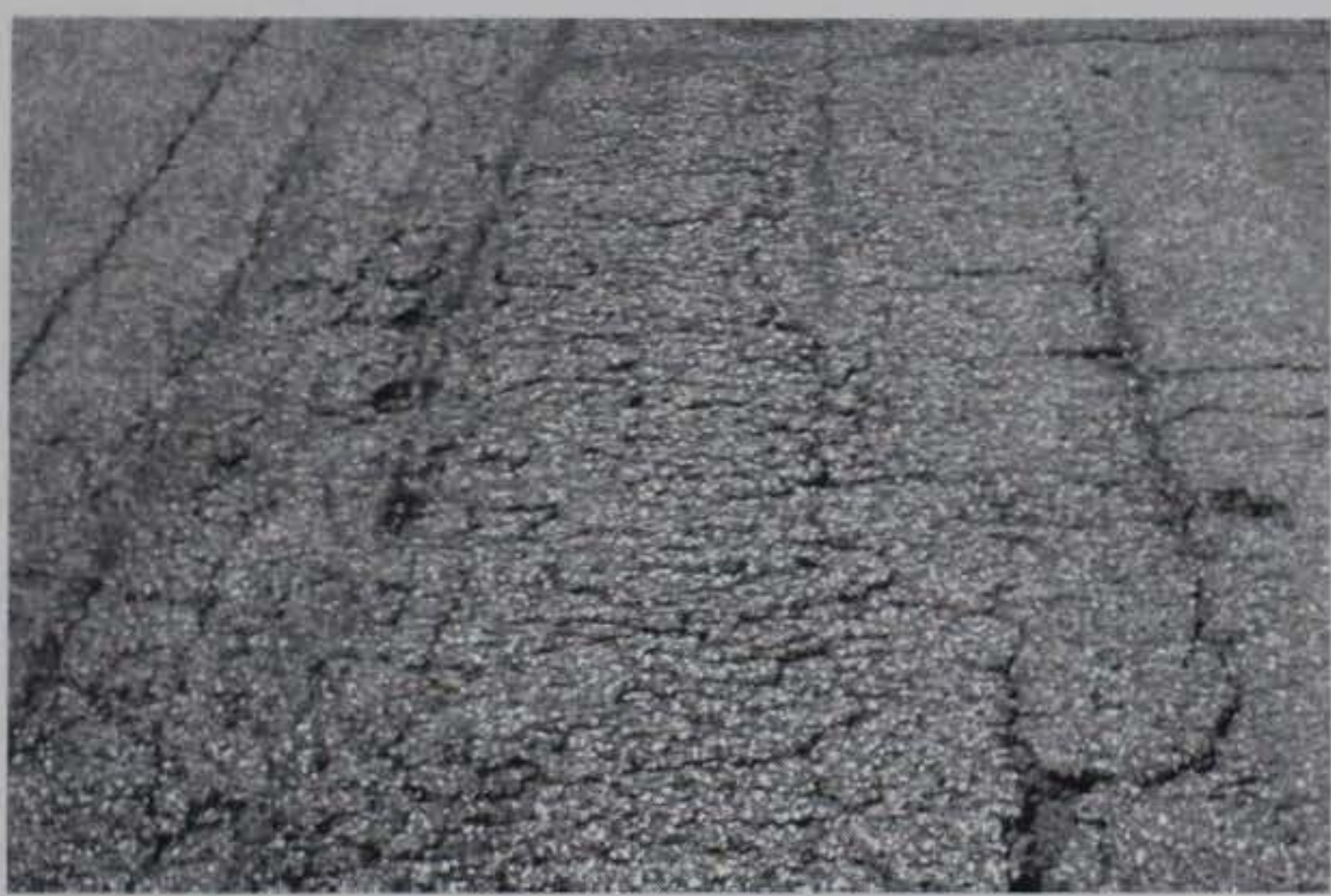

Figure 19b. Medium-Severity Weathering and Raveling.

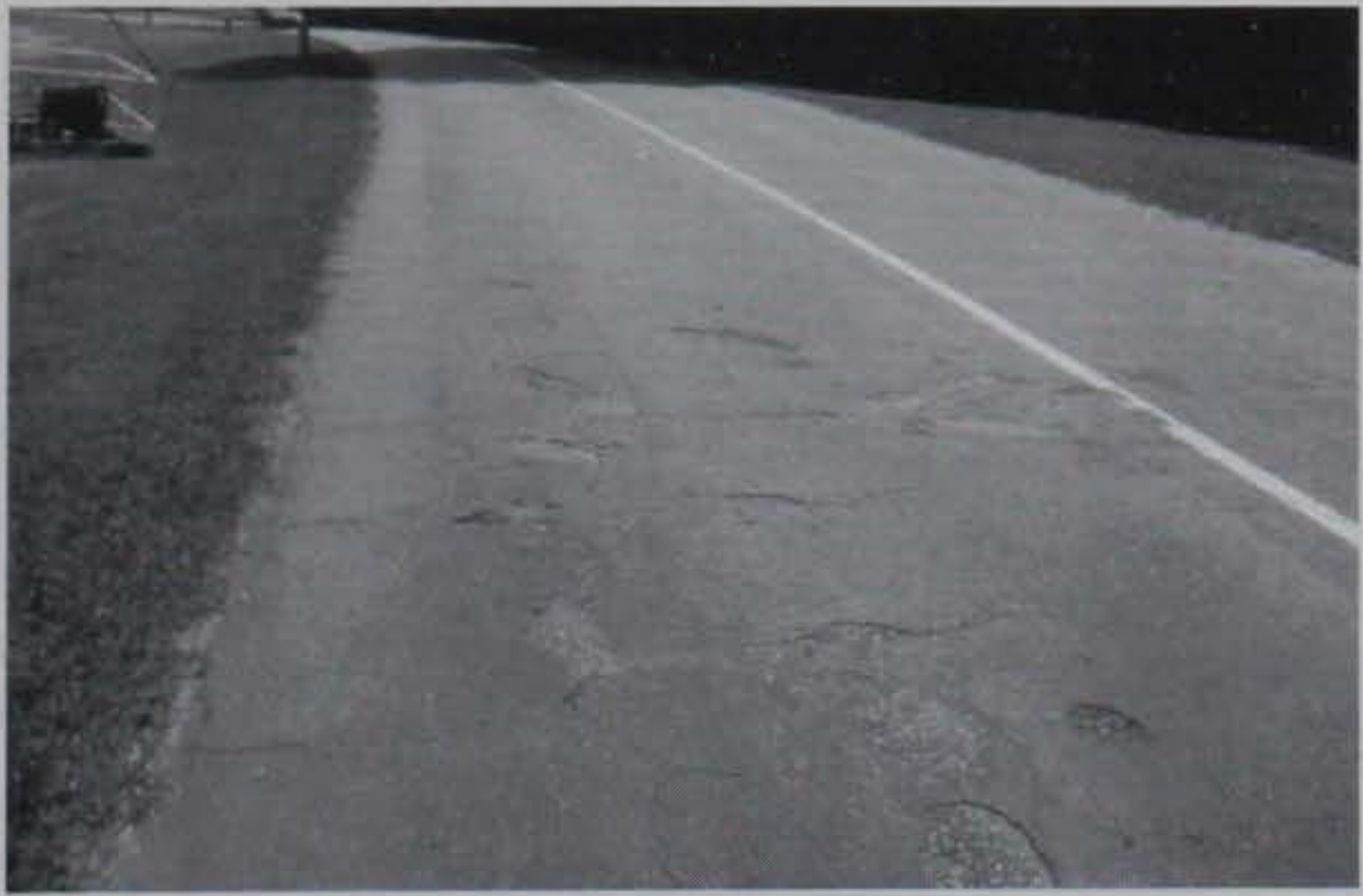

Figure 19c. High-Severity Weathering and Raveling. 


\section{References}

Shahin, M.Y., and J.A. Walther, Pavement Maintenance Management for Roads and Streets Using the PAVER System, Technical Report (TR) M-90/05ADA227464 (U.S. Army Construction Engineering Research Laboratory [USACERL], July 1990).

Shahin, M.Y., and S.D. Kohn, Pavement Maintenance Management for Roads and Parking Lots. TR-M-294/ADA110296 (USACERL, October 1981).

Technical Manual (TM) 5-623, Pavement Maintenance Management (Headquarters, Department of the Army, 1982). 